\title{
Identification of Thermal Abnormalities by Analysis of Abdominal Infrared Thermal Images of Neonatal Patients
}

by

Ruqia Nur

A thesis submitted to the Faculty of Graduate and Postdoctoral affairs in partial fulfillment of the requirement for the degree of

Master of Applied Science

in

Biomedical Engineering

Carleton University

Ottawa, Ontario

(C) 2013

Ruqia Nur 


\section{Abstract}

Necrotizing enterocolitis (NEC), is a devastating inflammatory disease of infants for which there is no cure and exact causes remain unknown. Diagnoses are limited to radiographic findings and in most institutions Modified Bell's Criteria is used, neither are capable of reliable early detection. In this thesis, a novel method of abdominal infrared thermal imaging is proposed that allows direct measurements of skin temperature, which are capable of unveiling thermal abnormalities that may indicate intestinal inflammation characteristic of NEC. Abdominal thermal symmetry analysis was performed, results obtained from the 20 normal and the 9 NEC affected infants were statistically compared. A higher degree of thermal asymmetry was seen with the NEC group in comparison to the Normal group, notably when image enhancement techniques were done. We are hopeful that this new non-contact, non-ionizing method may potentially offer an early diagnostic tool. 


\section{Acknowledgements}

I would like to thank my supervisor, Dr. Monique Frize, who took a chance on me and has guided me throughout my thesis with patience and support. I attribute the attainment of my Master's degree to the encouragement and knowledge provided by her, without which I would not have been driven to complete this thesis. I would like to also thank my co-supervisor Dr. Bariciak for her support and knowledge. I have also been blessed with friendly and cheerful colleagues and departmental support staff that provided an environment that not only made me feel comfortable, but that I enjoyed.

I would like to extend my deepest thanks to those whose support cannot be

forgotten, my family, friend, and especially my mother for their endless support in all my academic and personal endeavours.

Finally, I am thankful that I was in a point in my life where I was able to complete this thesis. This was a test of strength, endurance, and character for which I thank my creator for challenging me through this journey. 


\section{Table of Contents}

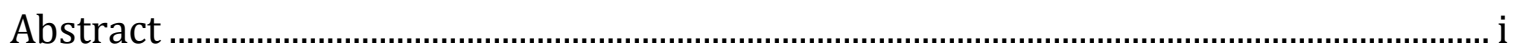

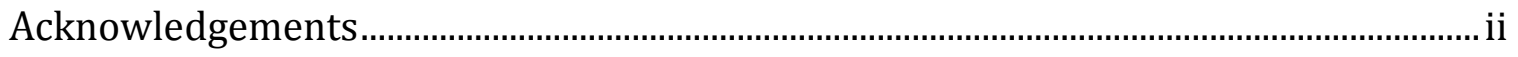

Table of Contents ..................................................................................................................

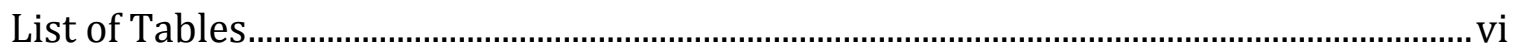

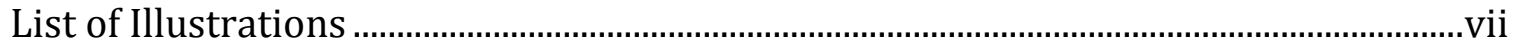

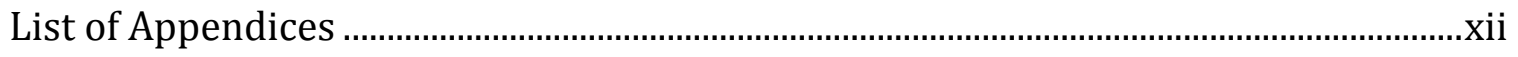

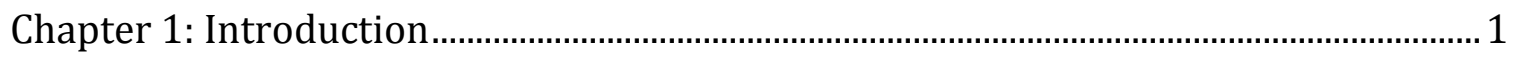

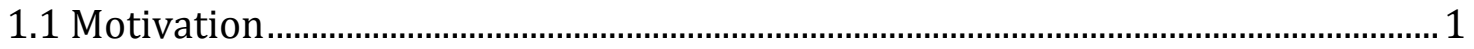

1.2 Thesis Objectives and Definition of the Problem....................................................... 3

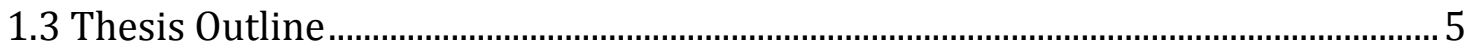

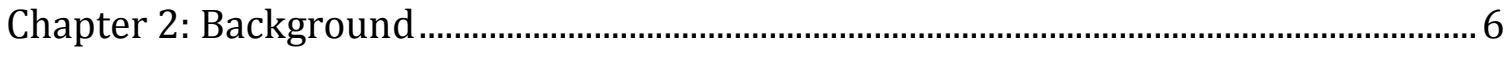

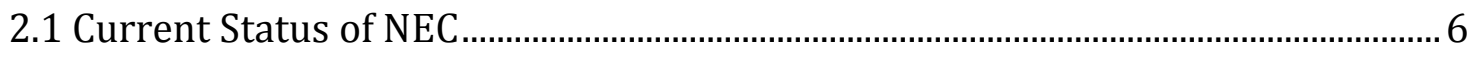

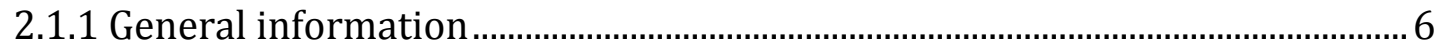

2.1.2 Technologies for the Detection of NEC ....................................................... 10

2.1.3 Rationale for detection of NEC using IR Thermal Imaging ............................ 12

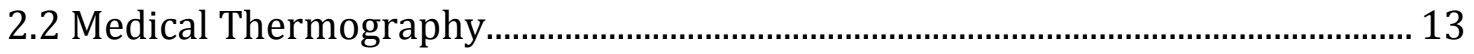

2.1.1 Overview of Medical Thermography ................................................................ 13

2.1.2 Medical Infrared Imaging Technology ……................................................... 15

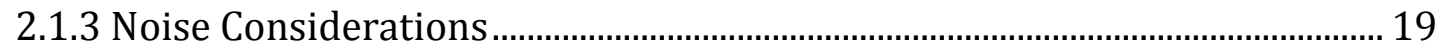

2.1.4 Clinical Infrared Thermal Imaging ................................................................. 21

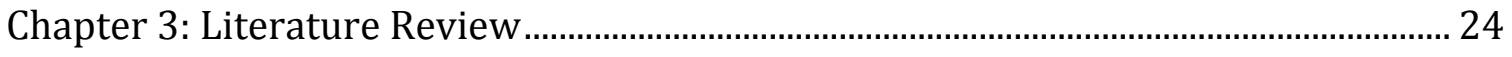

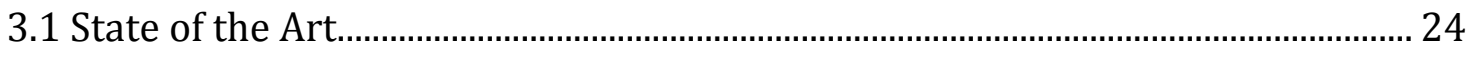

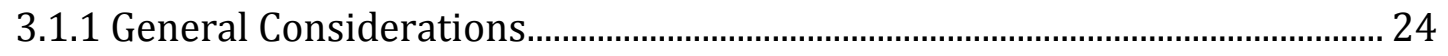


3.1.2 Statistical Analysis ...................................................................................... 26

3.1.3 Spatial Methods …………………………………………………………………. 29

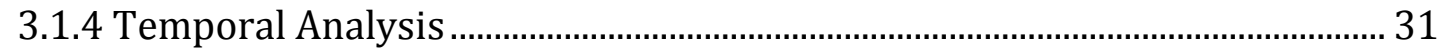

3.1.5 Image Processing Techniques ............................................................................. 34

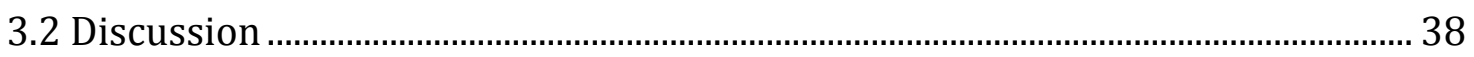

Chapter 4: Identification of Inflammation Associated with NEC Through Infrared

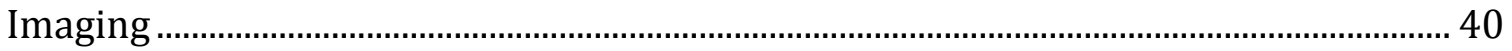

4.1 Methodology for Analysis of Abdominal Infrared Thermal Images..................... 40

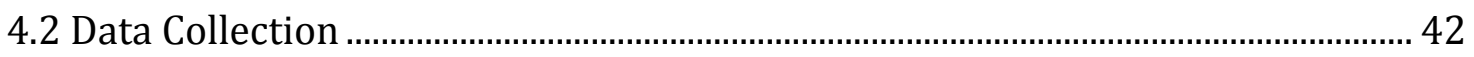

4.2.1 Equipment...................................................................................................... 42

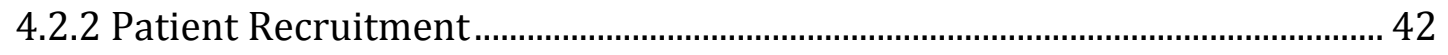

4.2.3 Imaging Protocol ................................................................................................. 43

4.2.3 Image Selection ....................................................................................................... 45

4.3 Thermal Image Processing Techniques....................................................................... 46

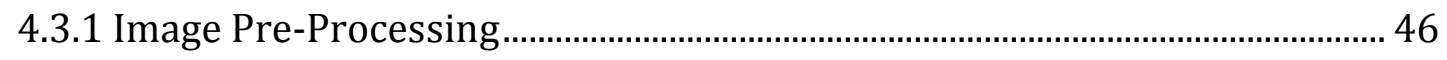

4.3.2 Image Enhancement …………………………………......................................... 48

4.3.3 Region of Interest Selection................................................................................. 56

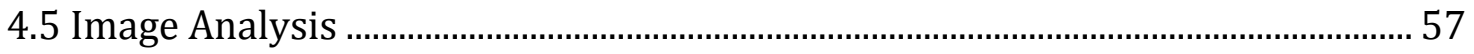

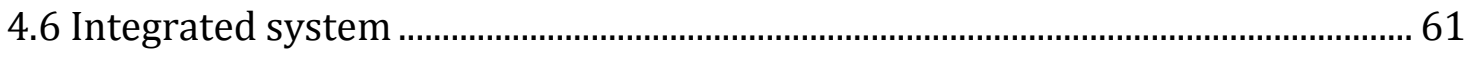

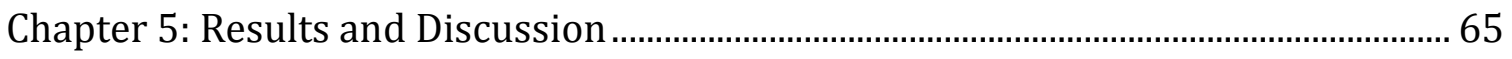

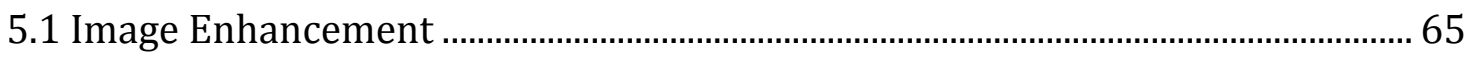

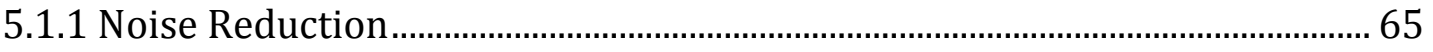

5.2.1 Contrast Enhancement................................................................................... 67

5.2 Data Analysis ............................................................................................................. 69

5.2.1 Lilliefors Test ...................................................................................................... 70 
5.2.2 Tests of statistical significance......................................................................... 74

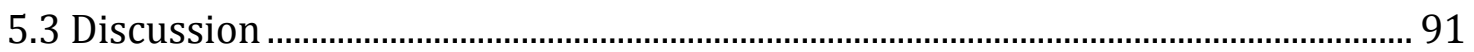

Chapter 6: Conclusion ................................................................................................... 94

6.1 Final Remarks.................................................................................................... 94

6.2 Contributions to Knowledge .................................................................................... 95

6.3 Future Work

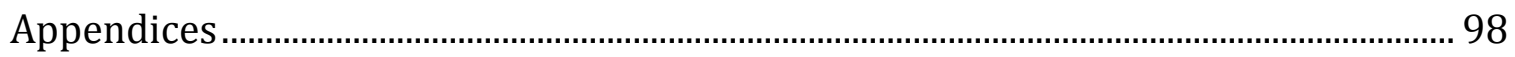

Appendix A .............................................................................................................. 98

A.1 Research Ethics Proposal .................................................................................... 99

A.2 CHEO Parent Information Sheet...............................................................................114

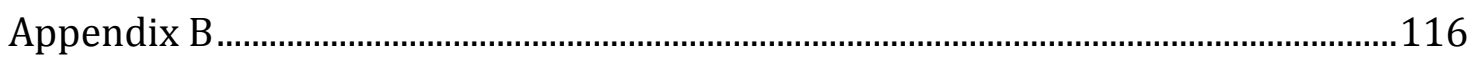

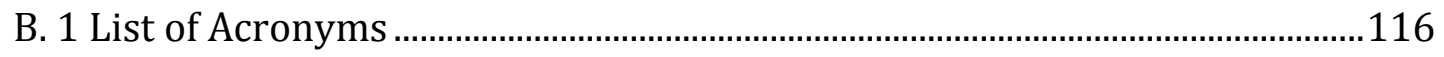

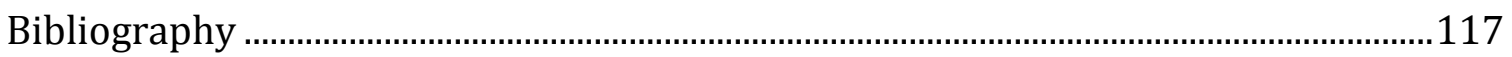




\section{List of Tables}

Table 2-1: Modified Bell's Staging Criteria for NEC. (Adapted from Walsh and Kliegmen [7], [25], [27])

Table 5-1: Results of Lilliefors test of normality for all 27 first order and simple statistical features extracted from original thermal images............................................ 71

Table 5-2: Results of Lilliefors test of normality computed for all 27 first order and simple statistical features extracted from enhanced thermal images. 72

Table 5-3: Results of Lilliefors test of normality for all 27 first order and simple statistical features calculated from the GLCM of original thermal images. 73

Table 5-4: Results of Lilliefors test of normality for all 27 first order and simple statistical features extracted from the GLCM of enhanced thermal images. 74

Table 5-5: The Wilcoxon Rank-Sum and Kruskal-Wallis tests performed for all 27 first order and simple statistical featured using original thermal images. The rank is based on the ascending order of p-values, when $\mathrm{h}=1$. 76

Table 5-6: The Wilcoxon Rank-Sum and Kruskal-Wallis tests performed for all 27 first order and simple statistical featured using enhanced thermal images. The rank is based on the ascending order of $p$-values, when $h=1$

Table 5-7: Results of the Wilcoxon Rank-Sum and the Kruskal-Wallis tests performed for all 27 first order and simple statistical features extracted from the GLCMs computed for original thermal images. 89

Table 5-8: Results of the Wilcoxon Rank-Sum and the Kruskal-Wallis tests performed for all 27 first order and simple statistical features extracted from the GLCMs computed for enhanced thermal images ......................................................... 90 


\section{List of Illustrations}

Figure 2-1: Pathophysiology of Necrotizing enterocolitis (adapted from [8], [6]) ....... 7 Figure 4-1: Example of how serial thermal images of the abdomen were captured. 44 Figure 4-2: This flow diagram depicts the four steps used to process, enhance, segment and analyze thermal images. ROIs available were the: whole, left, right, upper, lower, right upper quadrant (RUQ), right lower quadrant (RLQ), left upper quadrant (LUQ), and left lower quadrant (LLQ). First order and second order statistical features were computed for each ROI, and the differences between the upper-to-lower, left-to-right, and sum of quadrants-to-whole (QTW) were computed

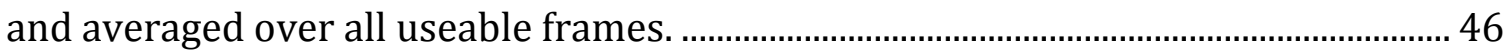

Figure 4-3: Example of a 3x3 kernel with even weighting ............................................ 50

Figure 4-4: Block diagram depicting the simulation performed to determine which filter was the best. The mean, median, and Weiner filters of size $3 \times 3,5 \times 5$, and $7 \times 7$ were compared. The mean square error was computed to evaluate performance and the filter with the lowest value was selected...... 51 Figure 4-5: (a) Depicts the semi-automated ROI selection of the whole and umbilicus regions. The centroid is determined based on the umbilicus region (b) The whole abdominal ROI is sliced based on the location of the centroid to further segment the whole abdomen into halves (c-d)and quadrants (e). 57

Figure 4-6: An example of the GLCM created from the $4 \times 4$ image I with 4 greylevels. The horizontal direction with distance 1 was used. 59

Figure 4-7: Flow diagram depicting the pre-processing, images enhancement, and analysis performed to create original and enhanced thermal images. After the pre- 
processing stage original images are indicated by dashed lines. First and second order thermal statistics were extracted from original and enhanced thermal images. Notice that original images were normalized when second order statistics were computed. The average of all U-to-L, L-to-R, and sum QTW differences of the statistical features extracted was then computed. 60

Figure 4-8: This GUI depicts the integrated system developed to perform computerized analysis of abdominal infrared images. 61

Figure 4-9: Initial thermal image before selection or processing, it was not normalized and no enhancement was performed. In this image it is evident that rotation to the right is required. The two cold spots on the top of the image are ECG electrodes. 62 Figure 4-10: The original thermal image was rotated to the right by 10 degrees. The image now appears aligned. 63

Figure 4-11: Example of the output directory when one image was used for thermal analysis. 64

Figure 5-1: Performance of mean, median, Wiener filter in removing white Gaussian Noise measured in MSE values. (a) An original thermal image that was normalized and with noise (b) - (d) Resulting images after noise reduction with the specified filters, only the best performing filter size of the three types tested is displayed..... 66 Figure 5-2: (a) Background removal using Otsu's Algorithm, performed on a normalized original thermal image (b) - (f) CLAHE with varying clip limits, performed on (a). Subtle changes are noted in (c) and (d), whereas striking 
differences are noticed in (e) and (d). These images were captured from an infant

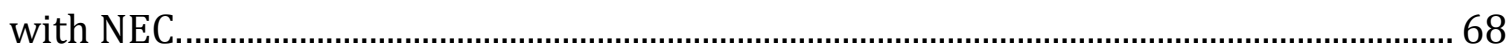

Figure 5-3: CLAHE (clip limit $=0.05$ ) performed on normalized original thermal images. (a) Images captured from an infant with NEC and (b-c) Normal infants ...... 69 Figure 5-4: Box plot for U-to-L difference of means $\left({ }^{\circ} \mathrm{C}\right)(0$-Normal, 1-NEC) from original thermal images. 77

Figure 5-5: Box plot for U-to-L difference of medians $\left({ }^{\circ} \mathrm{C}\right)(0$-Normal, 1-NEC) from original thermal images. 77

Figure 5-6: Box plot for U-to-L difference of Modes $\left({ }^{\circ} \mathrm{C}\right)$ (0-Normal, 1-NEC) from original thermal images. 78

Figure 5-7: Box plot for U-to-L difference of variances $\left({ }^{\circ} \mathrm{C}\right)(0$-Normal, 1-NEC) from original thermal images. 78

Figure 5-8: Box plot of the sum of QTW difference of means $\left({ }^{\circ} \mathrm{C}\right)(0$-Normal, 1-NEC) from original thermal images. 79

Figure 5-9: Box plot of the sum of QTW difference of medians $\left({ }^{\circ} \mathrm{C}\right)(0$-Normal, 1NEC) from original thermal images.

Figure 5-10: Box plot of the sum of QTW difference of modes $\left({ }^{\circ} \mathrm{C}\right)(0$-Normal, 1-NEC) from original thermal images. 80

Figure 5-11: Box plot of the sum of QTW difference of variances $\left({ }^{\circ} \mathrm{C}\right)(0$-Normal, 1NEC) from original thermal images. 80 Figure 5-12: Box plot of the sum of QTW difference of IQRs $\left({ }^{\circ} \mathrm{C}\right)(0$-Normal, 1-NEC) from original thermal images. 81 
Figure 5-13: Box plot of U-to-L difference of Means (0-Normal, 1-NEC) from

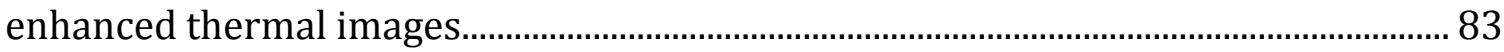

Figure 5-14: Box plot of U-to-L difference of Medians (0-Normal, 1-NEC) from

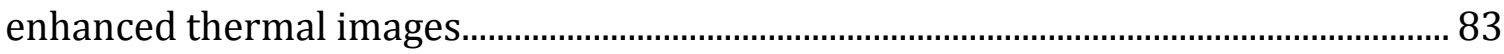

Figure 5-15: Box plot of U-to-L difference of Modes (0-Normal, 1-NEC) from

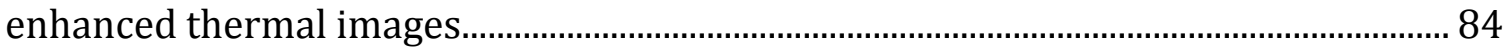

Figure 5-16: Box plot of U-to-L difference of Variances (0-Normal, 1-NEC) from

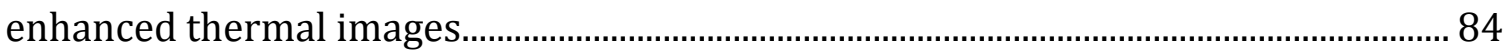
Figure 5-17: Box plot of U-to-L difference of Skewness (0-Normal, 1-NEC) from

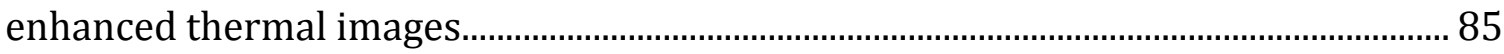
Figure 5-18: Box plot of U-to-L difference of Kurtosis (0-Normal, 1-NEC) from

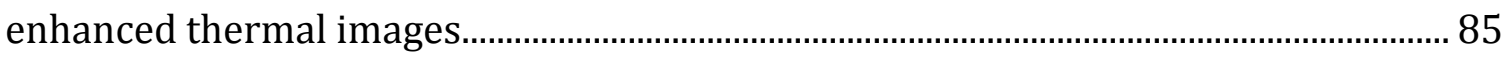
Figure 5-19: Box plot of U-to-L difference of IQRs (0-Normal, 1-NEC) from enhanced thermal images. 86 Figure 5-20: Box plot of Sum of QTW differences of Means (0-Normal, 1-NEC) from

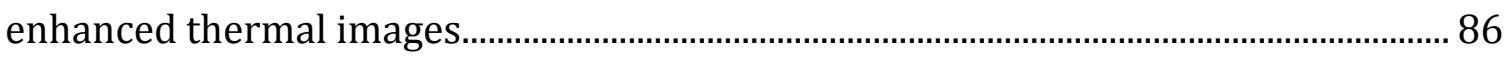
Figure 5-21: Box plot of Sum of QTW differences of Medians (0-Normal, 1-NEC) from

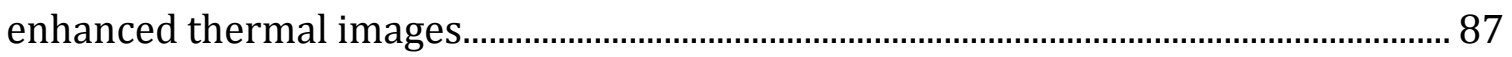
Figure 5-22: Box plot of Sum of QTW differences of Modes (0-Normal, 1-NEC) from

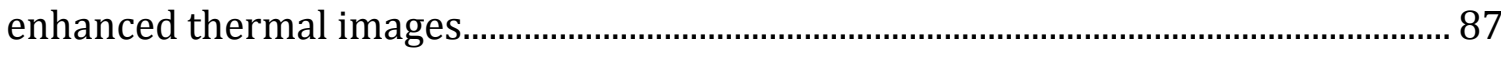
Figure 5-23: Box plot of Sum of QTW differences of Variances (0-Normal, 1-NEC)

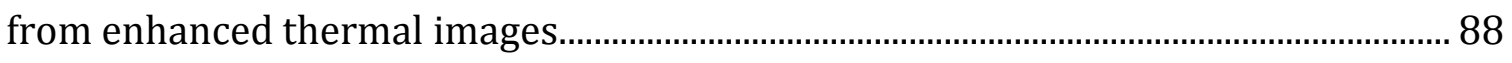


Figure 5-24: Box plot of Sum of QTW differences of IQRs (0-Normal, 1-NEC) from enhanced thermal images................................................................................................ 88 


\section{List of Appendices}

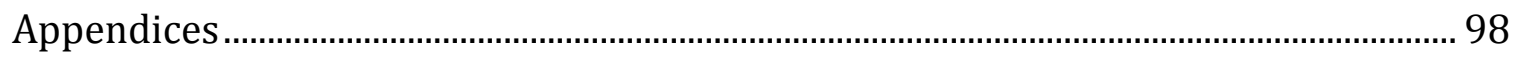

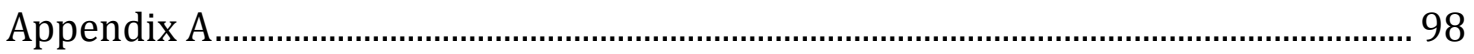

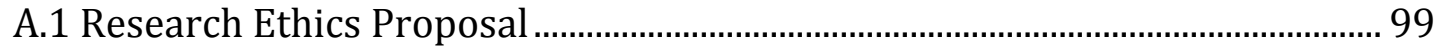

A.2 CHEO Parent Information Sheet ……....................................................................114

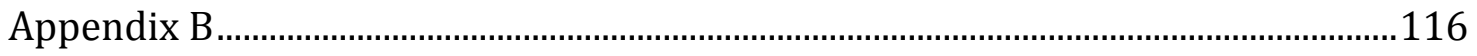

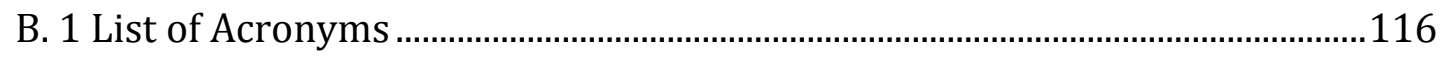




\section{Chapter 1: Introduction}

\subsection{Motivation}

Necrotizing Enterocolitis (NEC) is the most common and devastating inflammatory disease affecting the gastrointestinal (GI) tract of neonates. In statistical analysis of the population at risk, NEC has been found to occur in roughly $7 \%$ of preterm infants ( $<37$ weeks gestational age) with very low birth weight (VLBW) $(<1500 \mathrm{~g})$ [1]. Of those patients that don't require surgery the mortality rate is $15-25 \%$, and heightens to $40 \%$ with those that require surgery [2]. Despite decades of research, preventative methods remain elusive and the exact mechanisms of the disease remain unknown [3]. This complex multi-factorial disease typically presents within the first two weeks of life. NEC poses a significant clinical challenge because early signs and symptoms are subtle and nonspecific. The onset of disease can trigger an inflammatory cascade; when left untreated rapid deterioration of the body occurs, leading to the necrosis of intestinal tissue. In severe cases, intestinal perforation, sepsis, and even death can result [4]. Available treatments are often inadequate and no preventative methods are currently agreed upon [5], [6]. With increased survival rates of Very Low Birth Weight (VLBW) infants owed to medical advancements, the population at risk of developing NEC is sure to increase [7], [8].

Several technologies have been explored in search for non-invasive tools to detect and monitor the evolution of NEC. Abdominal radiography and sonography are the current imaging modalities used for the evaluation of the disease [7], [9]. These methods are not suitable for the early detection of NEC and interpretations of these 
images are not always straightforward. Still, in most institutions modified Bell's criteria is primarily used for diagnosis of NEC [7]. This four stage criteria is based on signs and symptoms that are not specific to NEC. It is even more challenging to diagnose stage 1; a definitive diagnosis is specified by stage 2 or higher [6], [7]. The need for an alternative method capable of early and reliable detection of NEC is urgent. Interestingly, the inflammation and pain associated with NEC is experienced by infants before the appearance of significant signs on x-ray images [10], [11].

When detecting infrared radiation emitted from the body, infrared imaging can measure the temperature distribution of the skin.

Infrared imaging is used to measure the temperature distribution of the skin, by capturing the infrared radiation emitted from the body [12]. Inflammation and fever can be recognized through touch alone, by locating areas with higher local temperatures. Pathologies detected using thermography has been generally associated with changes in blood perfusion [13]. Thermal abnormalities can be identified by asymmetrical thermal patterns, and/or the presence of hot and/or cold spots. IR camera technology is rapidly evolving, non-invasive, non-contact, low cost and a wide range of applications have been used to detect thermal abnormalities in a host of medical conditions. These applications include neurology [14], vascular disorders [15], breast cancer detection [16], [17], rheumatic diseases [18], [19]and has been strongly encouraged for neonatology [20].

NEC is an inflammatory disease primarily targeting the intestine. In this region, inflammation occurs which can drastically increase local temperatures. With possibility that abdominal infrared imaging may be suitable for the detection of inflammation 
associated with NEC [20], and because inflammation is an early symptom of NEC, its potential as an earlier diagnostic tool is plausible [10], [11].

\subsection{Thesis Objectives and Definition of the Problem}

The quantification of medical infrared images has been attempted by many researchers. Many medical applications have been developed, although the detection of breast cancer remains the most widely reported. No one so far has proposed to apply comprehensive computerized techniques for the assessment of static abdominal infrared thermal images of infants suffering with NEC. But, the notion of identifying NEC using infrared images has also been proposed by Rice et al. [21]. This idea is propelled by the fact that the intestinal inflammation and thermal dysfunction associated with NEC is well understood, and present prior to the appearance of significant signs on x-ray images [10], [11], which is the current method of diagnosis. This fact, in conjunction with the urgent need for a novel method that is non-invasive, non-ionizing and non-contact, fuels the need to explore abdominal infrared thermal imaging as an alternative imaging modality.

In this thesis two major components of digital image processing of thermal infrared images have been addressed, while automation was adopted where feasible.

The first objective was to quantify abdominal thermal symmetry through the computerized assessment of static thermal images. Comparisons were performed of first order and simple statistical measurements of contralateral symmetric regions of the abdomen, chosen among those used in literature. Thermal symmetry analysis was also conducted for the grey-level co-occurrence matrix (GLCM) computed for the abdominal thermal images, providing second order statistical features. 
Statistical tests were then performed to compare the distribution of these first and second order statistical features for the two groups (0-Normal and 1-NEC). The results were further analysed to observe if any feature indicated a higher degree of abdominal thermal asymmetry and for the associated group.

The first task was to find the correct and precise selection of the abdominal region of interest (ROI). The legitimacy of thermal symmetry results relies heavily on precise selection of contralateral symmetric regions of skin. To the best of our knowledge, segmentation limited to the abdomen has not been investigated. Manual selection of the abdomen according to anatomical landmarks was attempted. Prior, image pre-processing was required to correct the orientation and normalize the colour scale.

The second objective was to develop automated image enhancement techniques appropriate for medical infrared images. The goal was to improve the interpretability of images and unveil details that were previously hidden, while maintaining important information. To measure the efficacy of these methods, results of thermal symmetry analysis were compared for the original and enhanced thermal images.

In addition, it was planned to do an integration of all these components into a full system. This system was designed to facilitate effortless regeneration of thermal analysis with the option of using different processing parameters. The original size of the ROI was preserved, the user was also able to easily translate it to correct for motion in subsequent images of a thermal sequence. 


\subsection{Thesis OUTLINE}

This section provides an overview of later discussions.

Chapter 2 discusses in more detail the disease and the technologies currently used for detection. The necessary background information to understand key concepts of thermal imaging and its limitations are also discussed.

Chapter 3 provides a comprehensive literature review on the quantitative assessment of medical thermal images. Then limitations of previous literature and the need for a resolution are highlighted.

Chapter 4 defines the problem tackled in this thesis. The first part explains the necessary requirements to perform quantitative analysis of the thermal distribution of abdominal skin and assess symmetry. Discussion then shifts to the image processing techniques incorporated in this study, to reduce the limitations encountered in current literature surrounding the processing of medical infrared images.

Chapter 5 describes in detail the approach used in this thesis. The processing of thermal sequences and the significance of proper image selection are discussed. Results of statistical analysis performed to compare the results of thermal symmetry analysis for NEC and normal data are presented. This analysis was repeated to compare the results of thermal symmetry testing of the two groups (0Normal and 1-NEC), obtained from the original and enhanced thermal images.

A summary of these results can be found in Chapter 6, along with the contributions to knowledge and future work. 


\section{Chapter 2: Background}

This section aims to provide background information required to understand the rationale behind the use of medical thermography for the identification of inflammation associated with NEC. Section 2.1 will provide an overview of the disease, currently used technologies, and the inflammatory process involved in NEC. In section 2.2 the focus shifts to the physics of infrared imaging, overview of the technology and the practice of medical thermography.

\subsection{CuRRENT Status OF NEC}

\subsubsection{GENERAL INFORMATION}

Despite decades of research, preventative methods remain elusive and the exact mechanisms of the disease are unknown. For this reason many intestinal conditions are termed "Necrotizing enterocolitis", but vary in their pathogenesis (cause of disease) and therefore in methods of prevention and treatment [3], [22], [23]. The focus of this work is on the "classical" form of NEC, defined as an inflammatory intestinal condition in premature infants ( $<37$ weeks gestational age); differing from that seen in full term infants or in cases of spontaneous intestinal perforation [11]. Approximately $90 \%$ of neonates with NEC are preterm; the risk factor is inversely proportional to the gestational age and birth weight. The prevalence of NEC is 7 to $10 \%$ in the very low birth weight (VLBW) preterm infants $(<1500 \mathrm{~g})$. With the advancement of obstetric and neonatal care the survival rate of preterm infants $(<1500 \mathrm{~g})$ with VLBW is bound to increase; therefore so is the population at risk of NEC [5]. 


\section{Pathophysiology}

Although the pathophysiology is not fully understood, many associated risk factors are known, see Figure 2-1. A known common predisposing risk of preterm infants is intestinal immaturity, which can be characterized as: intestinal motility and absorption, intestinal barrier function, immune response, and circulatory regulation [5]. Additional predisposing risk factors attributed in the development of NEC include: genetic susceptibility, abnormal intestinal microbiota, feeding insult and compromised blood flow [24]. These risk factors, during the early prenatal period, can trigger an inflammatory cascade; if left undetected this may lead to the rapid deterioration of health culminating in necrosis of tissue. In severe cases, intestinal perforation, sepsis and even death can result [4]. It is said that NEC develops after birth and does not appear in stillborn infants [5].

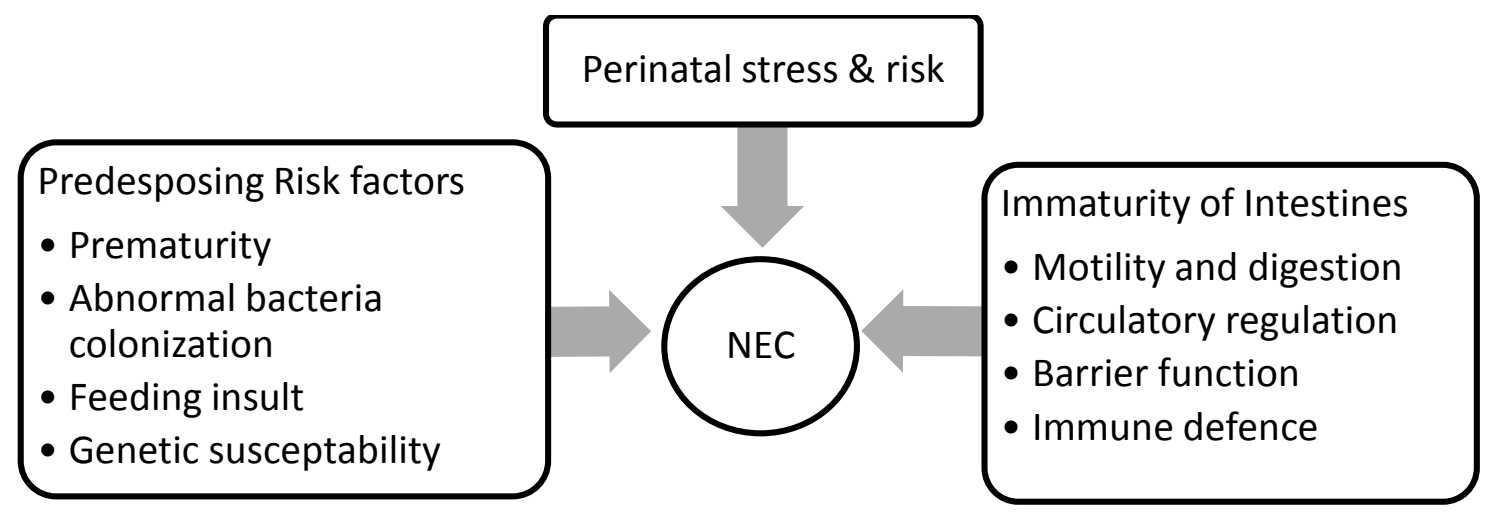

Figure 2-1: Pathophysiology of Necrotizing enterocolitis (adapted from [8], [6])

\section{Clinical Presentation}

This complex multi-factorial disease typically presents within the first two weeks of life. NEC primarily affects the gastrointestinal (GI) tract; in the worst cases a profound systemic impact is also noted. The onset is sudden and often appears 
within hours or days following a period of feeding intolerance [6]. NEC poses a significant clinical challenge because early signs and symptoms are subtle and nonspecific. Initial clinical presentation of the disease includes: feeding intolerance, bloody diarrhea, temperature instability, lethargy, apnea, bradycardia, hypotension, absent bowel sounds, decreased peripheral perfusion, abdominal distention or tenderness, respiratory distress, and on occasion the existence of a right lower quadrant mass [6], [25]. Laboratory indicators of NEC typically include metabolic acidosis, respiratory acidosis, thrombocytopenia, and neutropenia [23]. Radiographic images are used to assess the progression of the disease. Radiographic evidence of NEC is indicated by the presence of pneumatosis intestinalis (presence of air in-between the layers of the bowels) and in advanced cases pneumoperitoneum (indicates ongoing bowel perforation) [7], [8], [23], [25].

\section{Diagnosis}

The diagnosis of NEC in many institutions relies heavily on the combination of clinical, laboratory, and radiological findings. A reliable universal diagnostic criterion has yet to be established, which makes diagnosis difficult. Bell et al. in 1978 developed a staging system that provides a systemic description of NEC [26]. This system has since been refined. Now most institutions rely heavily on Modified Bell's Staging Criteria for NEC. This three stage criteria is summarized in Table 2-1. 
Table 2-1: Modified Bell's Staging Criteria for NEC. (Adapted from Walsh and Kliegmen [7], [25], [27])

\begin{tabular}{|c|c|c|c|}
\hline Stage & $\begin{array}{l}\text { Systemic } \\
\text { Findings } \\
\end{array}$ & $\begin{array}{l}\text { Abdominal } \\
\text { Findings } \\
\end{array}$ & $\begin{array}{l}\text { Radiographic } \\
\text { Findings }\end{array}$ \\
\hline $\begin{array}{l}\text { I } \\
\text { Suspected }\end{array}$ & $\begin{array}{l}\text { Apnea and } \\
\text { bradycardia, } \\
\text { temperature } \\
\text { instability, } \\
\text { lethargy }\end{array}$ & $\begin{array}{l}\text { Poor feeding, } \\
\text { Gastric residuals, } \\
\text { occult blood in } \\
\text { stool, mild } \\
\text { abdominal } \\
\text { distention }\end{array}$ & $\begin{array}{l}\text { Normal gas pattern } \\
\text { or mild ileus } \\
\text { (bowel } \\
\text { obstruction) }\end{array}$ \\
\hline $\begin{array}{l}\text { IIA } \\
\text { Definite, } \\
\text { mildly ill }\end{array}$ & Same as above & $\begin{array}{l}\text { Grossly bloody } \\
\text { stools, prominent } \\
\text { abdominal } \\
\text { distention, absent } \\
\text { bowel sounds }\end{array}$ & $\begin{array}{l}\text { Ileus gas pattern } \\
\text { with one or more } \\
\text { dilated loops and } \\
\text { focal pneumatosis }\end{array}$ \\
\hline $\begin{array}{l}\text { IIB } \\
\text { Definite, } \\
\text { moderately ill }\end{array}$ & $\begin{array}{l}\text { Same as above, } \\
\text { plus } \\
\text { Thrombocytopenia } \\
\text { and mild } \\
\text { metabolic acidosis }\end{array}$ & $\begin{array}{l}\text { Abdominal wall } \\
\text { edema with } \\
\text { palpable loops and } \\
\text { tenderness }\end{array}$ & $\begin{array}{l}\text { Widespread } \\
\text { pneumatosis, } \\
\text { ascites, portal- } \\
\text { venous gas }\end{array}$ \\
\hline $\begin{array}{l}\text { IIIA } \\
\text { Advanced, } \\
\text { severely ill, } \\
\text { Intact bowel }\end{array}$ & $\begin{array}{l}\text { Same as above, } \\
\text { plus acidosis, } \\
\text { oliguria, } \\
\text { hypotension, } \\
\text { coagulopath }\end{array}$ & $\begin{array}{l}\text { Worsening wall } \\
\text { edema, erythema } \\
\text { and induration }\end{array}$ & $\begin{array}{l}\text { Prominent bowel } \\
\text { loops, worsening } \\
\text { ascites, no free air }\end{array}$ \\
\hline $\begin{array}{l}\text { IIIB } \\
\text { Advanced, } \\
\text { severely ill, } \\
\text { perforated bowel }\end{array}$ & $\begin{array}{l}\text { Above, plus septic } \\
\text { shock, } \\
\text { deterioration in } \\
\text { laboratory } \\
\text { values and vital } \\
\text { signs }\end{array}$ & $\begin{array}{l}\text { Perforated } \\
\text { bowel }\end{array}$ & Pneumoperitoneum \\
\hline
\end{tabular}

\section{Management and Outcomes}

Given that the exact cause of NEC is unknown, effective treatment plans and methods of prevention are challenging. Currently, no preventative strategies are agreed upon and treatments are often inadequate and of a supportive nature. Once an infant is suspected to have NEC, different feeding strategies are explored, including cessation of feedings and gas is released from the stomach [1], [28] . Nutrition is provided through intravenous fluid and rounds of antibiotics are 
prescribed to fight infections that may be present. If health deteriorates and ongoing perforation or necrosis (death) of tissue is observed, bowel resection surgery is performed [6], [25]. Delay and inefficient treatment can lead to a worsened prognosis necessitating a surgical intervention. Timely intervention is crucial for the survival of the infants and the reduction of long-term morbidity that may result [1], [6], [25].

\subsubsection{TECHNOLOGIES FOR THE DETECTION OF NEC}

Currently, multiple technologies are available to detect and monitor the later stages of NEC. In this study we only discuss non-invasive methods, more specifically imaging modalities. Although endoscopy and colonoscopy produce clear images of the intestinal walls, they are not used due to the increased risk of damaging already weakened intestines.

\section{Radiographic Imaging}

The current standard imaging modality for the evaluation of NEC is abdominal radiography. A criterion for the interpretation of x-ray images has been well established. If an infant is suspected of NEC, an abdominal x-ray is captured using a vertical or horizontal beam with the infant in the supine or decubitus position. Signatures of x-ray images indicative of NEC include dilated bowel loops, pneumatosis intestinalis (gas in the bowel wall) and pneumoperitoneum (air leakage from the intestines by perforation) [8], [9]. These signs are clear indicators for the urgent need for a surgical intervention. This technique indicates the status of health for a single point in time and is not suitable for continuous bedside monitoring, which is necessary due to the fulminant nature of the disease. In some 
instances ongoing perforation or necrosis of tissue is not detected. In addition, the amount of radiation exposure is worrisome, which makes computed tomography a less desirable avenue to increase the performance of radiography.

\section{Sonography}

In some instances, intestinal perforation can develop without radiographic detection of free air, posing a significant challenge. The usefulness of ultrasonography $(\mathrm{U} / \mathrm{S})$ as a detection method has been investigated in the absence of gas in the abdomen. Infants with no clinical symptoms, or infants with a gasless abdomen and deteriorating health, were shown to have U/S findings of ascites (fluid filling the space between the abdominal lining and organs) and debris in fluid consistent with perforation that occurs with NEC [29]. In another study, infants with the same symptoms were imaged; here ascites and hyperemia (increased blood flow) of bowel loops were noticed suggesting the need for surgical intervention [30].

A limitation of abdominal $\mathrm{U} / \mathrm{S}$ is that evaluation is difficult when a considerable amount of gas is present [9]. Therefore, $\mathrm{U} / \mathrm{S}$ is a useful tool when imaging is done on gasless distended abdomens, which represents a small population of NEC affected infants [29]. In addition, $\mathrm{U} / \mathrm{S}$ requires direct contact with the abdomen, which should be avoided considering the significant pain and tenderness that is typically experienced by NEC affected infants.

\section{Magnetic Resonance Imaging (MRI)}

One study assessed the potential of abdominal MRI in the detection of NEC in preterm infants [31]. The presence of pneumatosis intestinalis was successfully 
identified in those who required surgical interventions; pneumatosis intestinalis was not seen in infants who were only suspected of NEC. Although this method was able to detect some form of NEC, it did not outperform abdominal radiography, on the subject of early detection. Another limitation is the need to transport unstable infants from the neonatal intensive care unit (NICU) to the MRI unit and the amount of noise produced. The clinical usefulness of MRI for the early detection and monitoring of NEC is not yet convincing.

\subsubsection{RATIONALE FOR DETECTION OF NEC USING IR THERMAL IMAGING}

Anbar [13] states that "abnormal behaviour of skin temperature can be manifest in two principal modalities: (1) pathological changes in the spatial distribution of temperature over the skin surface, (2) pathological changes in the dynamic temperature behaviour, i.e., warming, cooling, or periodic cooling of a given sub area of skin." There are a number of pathological conditions that are associated with abnormal thermal behaviour.

In NEC the inflammatory cascade characteristic of the disease may be observed and attributed as the main cause of thermal abnormality. This is because inflammation is a mechanism by which local increases in heat may be generated by the site of inflammation; which is also observed in the case of infection and wound healing. Since inflammation targets the intestines, the presence of thermal abnormalities may be realised through the evaluation of the temperature distribution of abdominal skin. Infrared imaging is capable of accurately capturing the thermal distribution of skin and could potentially offer an early diagnostic imaging modality, since inflammation is one of the earliest symptoms of the disease [10], [11]. The exploration of IR imaging is further propelled by the urgent need for the innovation of NEC technologies. 


\subsection{MEDiCAL THERMOGRAPHY}

\subsubsection{OVERVIEW OF MEDICAL THERMOGRAPHY}

All objects above at a temperature absolute zero $\left(-273.15^{\circ} \mathrm{C}\right)$ emit energy in the form of electromagnetic radiation, also termed thermal radiation or infrared radiation [12], [32]. The bulk of energy emitted by a body is within the infrared part of the electromagnetic spectrum. Infrared thermography is a measurement of the temperature distribution of a surface using the infrared radiation emitted by the body within the $0.8 \mu \mathrm{m}$ to $1 \mathrm{~mm}$ [12], [33]. For medical imaging, the wavelengths of importance include midwave infrared (2-5 $\mu \mathrm{m})$, and long-wave infrared (8-12 $\mu \mathrm{m})$ [33]. An infrared camera is a non-invasive tool that is capable of detecting thermal radiation and generating a 2-dimensional image representing the temperature distribution of a surface; size remains dependent upon the field of view [12].

The temperature of an object determines the resulting spectral emission. The temperature and emissivity of an object determines the amount of radiation detected. Emissivity is a measure of how much radiation an object emits in comparison to an object with no reflectivity, at the same temperature [12]. An ideal blackbody absorbs all incident radiation that falls upon it, and when in thermal equilibrium, it radiates a continuous spectrum according to Plank's Law. The total emissive power of a blackbody can be determined by integrating Plank's law over all wavelengths; known as the Stefan-Boltzmann's law:

$$
E_{b}=\sigma T^{4}\left[\mathrm{~W} / \mathrm{m}^{2}\right]
$$


where, $\sigma\left(5.6705 \times 10^{-8} \mathrm{~W} / \mathrm{m}^{2} \cdot \mathrm{K}^{4}\right)$ is the constant of proportionality, also termed the Stefan-Boltzmann constant, and $\mathrm{T}$ is the temperature (K). An ideal blackbody is also a perfect emitter and has an emissivity of 1 , which is not true of real surfaces. For this reason the Stefan-Boltzmann's law has been modified to account for the emissivity $(\epsilon)$ :

$$
E=\epsilon \sigma T^{4}\left[\mathrm{~W} / \mathrm{m}^{2}\right]
$$

Interestingly, it has been found that the peak emissive power is observed at approximately $9.5 \mu \mathrm{m}$ and is significant in the $2-5 \mu \mathrm{m}$ (midwave infrared), and 8-12 $\mu \mathrm{m}$ (long-wave infrared) wavelengths in comparison to the rest of the spectrum [33]. Also, the human skin in the $8-12 \mu \mathrm{m}$ range has an emissivity of 0.97 to 0.98 ; which permits accurate temperature measurements in this range [13], [34]. Although measurement in the 3 to $5 \mu \mathrm{m}$ range yields a high emissive power in comparison to the 8 to $12 \mu \mathrm{m}$, reflectivity is said to be highest in the $10-15 \%$ range [13]. Thus, accurate and reliable temperature measurements of human skin can be obtained with infrared imaging in the 8 to $12 \mu \mathrm{m}$ wavelength range.

In actuality, emissivity of a surface fluctuates according to the temperature, wavelength, and direction of the emitted radiation. However, it may be considered constant over a certain bandwidth, range of temperature, and cone of direction; reducing the complexity of calculations. An emissivity of unity and a reflectivity of zero are desired to obtain accurate temperature measurements. Under this constraint, the amount of correction required to consider the reflectivity of the skin is negligible particularly if images are captured in an environment which is slightly 
lower in temperature than that of the body, and if the angle of the measurement (or view) is less than $\pi / 4$ [12], [35].

\subsubsection{MEDICAL INFRARED IMAGING TECHNOLOGY}

In the 1970's, when use of clinical infrared imaging was first adopted, two different technologies were available: liquid-crystal contact thermography and noncontact blackbody imaging. Liquid-crystal technology uses a sheet of liquid crystals placed over the imaging area and the crystals change colour according to the temperature. The alternative, thermal imaging technology uses noncontact infrared sensors (detectors) to capture thermal radiation, by scanning the field of view on a pixel-bypixel basis. The energy captured by sensors (detectors) is converted into a temperature. The resulting 2D image is termed a thermogram, where each pixel is a digital value of temperature proportional to the amount of energy received [12].

Liquid-crystal technology has since become obsolete for medical purposes, due to low thermal resolution, low spatial resolution, and slow response time [13]. As well, thermal distributions captured using contacts are unreliable since the measuring device directly influences the temperature of the region being evaluated through direct contact [36]. The remainder of this discussion will be limited to non-contact thermal imaging systems based on its suitability for clinical use.

In the 1950's infrared cameras were bulky and required liquid nitrogen for cooling. In the 1990's new smaller self-cooled cameras that were fast scanning with high thermal and spatial resolutions were available [13].

Before specific characteristics of the most commonly used infrared detectors is discussed, it is necessary to define figures of merit used to compare the 
performance of various detectors. There are many technical parameters that are used to evaluate the performance of an IR detector and camera system [37], [38], [39], [40], [41].

The responsivity $(R)$ of a detector is the output quantity of the detectors $(S)$ (voltage or current) per watt of incident radiant energy and is a function of the wavelength of the incident radiation. However, responsivity does not account for the influence of noise on the output of detectors and for this reason it does not reflect the true performance of a camera.

The Noise Equivalent Power (NEP) measures the amount of incident radiation equivalent to the intrinsic noise of a detector. NEP is determined as the radiation level that results in a signal-to-noise ratio $(\mathrm{S} / \mathrm{N})$ of 1 . The NEP is also defined as the ratio of output noise to the responsivity (R). Since smaller values of NEP indicate a better performance, the reciprocal of this value termed the detectivity $\mathrm{D}$ is more convenient to use, given in $\mathrm{W}^{-1}$. NEP and $\mathrm{D}$ cannot be used to compare two different detectors because they are dependent on the bandwidth and detector area. To compare various detectors, the detectivity is normalized by the detector area and bandwidth to obtain the specific detectivity $\mathrm{D}^{*}$ (or D star); the responsivity per unit active area of a detector $\left(\mathrm{cm} \cdot H z^{\frac{1}{2}} \cdot W^{-1}\right)$. D allows for a more realistic comparison of detectors; the larger this value is, the better the detector element will be.

There are two classes of infrared detectors: photon detectors (photodetectors) and thermal detectors [38], [40], [42], [43]. Thermal detectors are sensitive to temperature variations and sense the heat generated by the absorption of incident 
radiation, which changes some electrical properties (resistance, capacitance or polarization, and voltage or current) to generate an output. This signal does not depend on the photonic nature of incident radiation; it is proportional to the direct change in temperature and is independent of wavelength. Consequently, these characteristics are constant over a wide range of bandwidths and over the electromagnetic spectrum. The three major types of thermal detectors commonly seen in infrared thermal imaging are discussed: bolometers, pyroelectrics and thermopiles.

A bolometer is a temperature-sensitive resistive element; typically the material used has a large temperature coefficient of resistance $\left(\alpha_{T}\right.$ given in $\left.\% /{ }^{\circ} \mathrm{K}\right)$, which produces a large increase in resistance with the absorption of incident radiation. This variable resistance is defined as:

$$
R=R_{0}\left(1+\alpha_{T} T_{d}\right)
$$

Where, $R_{0}$ is the resistance at a nominal temperature, $\alpha_{T}$ as defined above, and $\mathrm{T}_{\mathrm{d}}$ is the change in temperature $\left({ }^{\circ} \mathrm{K}\right)$. A thermocouple is composed of two different metals placed in close contact; when heated, the voltage at this junction changes. A thermopile is a series of thermocouples used to achieve greater sensitivity. The voltage responsible for the current flow between two junctions is directly proportional to the temperature difference; it is defined below:

$$
\Delta V=\alpha_{s} T_{d}
$$

where, $\alpha_{s}$ is the Seebeck coefficient $(\mu \mathrm{V} / \mathrm{K})$. A pyroelectric detector is composed of ferroelectric materials; here the polarity changes according to the temperature. The 
rate of temperature change is measured rather than the change in temperature itself. The output charge $Q$ and current $i$ with charge $Q$ is defined as:

$$
\begin{aligned}
& Q=p T_{d} \\
& i=p \frac{d \Delta T}{d t}
\end{aligned}
$$

where, $\mathrm{p}$ is the pyroelectric coefficient $\left(C / \mathrm{m}^{2} K\right)$.

A photon detector (quantum detector or photodector) is a semiconductor device that converts incident photons directly into an electrical signal. When photons within a certain range of wavelength fall upon the detector, the result frees electrons or charge carriers which can be detected as an electrical current or voltage. This excitation of charge carriers is proportional to the power of the incident radiation. These detectors are categorized based on their mode of operation: photoconductive, photovoltaic or photoemissive effects of the semiconductor materials used. Photoemissive detectors are not suitable for thermography since the infrared spectrum of interest was not being measured and will not be discussed.

Photoconductive detectors are composed of a material that changes electrical conductivity in proportion to the amount of incident radiation absorbed. In contrast, a photovoltaic detector requires an internal potential barrier, typically two dissimilar materials in close contact like a simple $p$ - $n$ junction. When struck by incident radiation, electrons are freed from the materials; these electrons that cross the potential barrier move more easily in one direction. The outcome is that one 
side is more negative with respect to the other, which can be measured as a current or voltage.

Thermal detectors are disadvantaged by their slow response time and lower detectivity in comparison to photon detectors. However, they can be used over a wide spectral range and are less expensive and cooling is not required. Photon detectors must operate at cryogenic temperatures to achieve accurate measurements and reduce the effect of noise. Photon detectors have a higher detection performance and response speed, but they are dependent on wavelength.

Historically, photovoltaic and photoconductive detectors have been preferred in thermography due to superior detectivity and rate of response. In addition, since they are wavelength dependent, the measurement spectrum can be toned to be suitable for medical imaging of skin. In recent times, microbolometer technology has advanced, and now has superior detectivity and response rate in the desired wavelength range. In addition, photon detectors have also increased their performance, especially those that utilize Quantum Well technology.

\subsubsection{NOISE CONSIDERATIONS}

The predominant noise sources for infrared detectors includes: $1 / \mathrm{f}$ noise, thermal noise (Johnson noise), shot noise, photon noise, and temperature noise [38], [41], [42], [44].

$1 / \mathrm{f}$ noise (or flicker noise) is a random fluctuation that occurs in most electronic devices; it increases as the frequency approaches zero (DC). The origin of this source is not easily explained and results in a variety of effects; it may be reduced if 
surfaces and contacts are prepared appropriately. The following is a general expression for the noise current:

$$
i_{n}=\left(\frac{K_{1} 2 I_{B}^{\alpha} \Delta f}{f^{\beta}}\right)^{\frac{1}{2}}
$$

where, $K_{1}$ is the detector dependent proportionality factor, $I_{B}$ is the bias current, $\Delta f$ is the observation bandwidth, $f$ is the frequency, $\alpha$ and $\beta$ are constants roughly 2 and 1.

Thermal Noise (or Johnson noise) is the fluctuation of a signal caused by thermal agitation of electrons in resistive elements. These electrons collide with atoms in the resistive element which creates energy. The thermal current noise $i_{j}$ is expressed as:

$$
i_{j}=\frac{\sqrt{4 k T \Delta f}}{R}
$$

where, $\mathrm{k}$ is the Boltzmann constant, $\mathrm{T}$ is the temperature in degrees Kelvin, and $\mathrm{R}$ is the electrical resistance in ohms.

Shot noise in current flow is attributed to the statistical nature of the generation of charge carriers (free electrons) in a photodetector when a photon strikes its surface. In a given time period, random fluctuations will occur in the generation of charge carriers caused by photon excitation. This noise source is not caused by imperfection of the detector or any electronics within the system. The resulting current that flows through the device follows a Poisson distribution; the current shot noise is expressed as:

$$
i_{n}=(2 e I \Delta f)^{1 / 2}
$$

where, $e$ is the electronic charge in coulombs, and $I$ is the total current. 
Generation-recombination noise (or G-R noise) is the result of variability in the generation, recombination and trapping of charge carriers in a semiconductor; which induces fluctuations in the resistance and conductivity of the material. Significant effects are noted when a bias current is applied, which is common in the case of photoconductors. G-R noise is frequency dependent and is considered to be a negligible contribution to the overall noise present at high frequency.

Photon noise arises from the random nature of the incident radiation. In other words, fluctuations are attributed to the discrete and random processes involved in the arrival rate (or emission rate) of photons. This is not owed to any defect of the detector or imaging system but of the signal of interest. Mentioned previously, photon noise is known to follow a Poisson distribution.

Temperature noise is one of the major limiting factors in thermography, caused by random fluctuations in the background temperature, i.e. a draft, direct sunlight, or ambient light.

As with all systems, the generation of noise is not limited to sources of the detector. Other sources that may significantly degrade the quality and accuracy of images include: thermal noise from amplification, quantization or readout noise in chargecoupled devices (CCD). However, careful design and integration of various components can reduce this noise level below that which is inherent to the detector.

\subsubsection{CLINICAL INFRARED THERMAL IMAGING}

Infrared imaging (Clinical infrared thermography) is a measurement of the temperature distribution of a surface corresponding to the infrared radiation emitted by the body [12]. This non-invasive, non-ionizing, and non-contact analytical 
tool is useful in evaluating the thermal behaviour of skin; it generates an image or a sequence of images. Clinical infrared imaging can therefore provide valuable information concerning the thermoregulatory process of the body. Although this imaging modality is only functional and cannot be used to provide an exact diagnosis of anatomical and/or structural pathologies, it is suggestive to the location and/or cause of thermal anomalies.

Medical thermography can be static or dynamic. Static thermography means that analysis is performed on an image captured at a single point in time. In dynamic thermography, a sequence of images of the same subject is analyzed, which makes comparison and temporal analysis of temperature distributions possible. Typically, a thermal stress test is conducted and serial imaging is performed until equilibrium is reached, providing more information as the physiological process is highlighted. The development of an appropriate imaging protocol is vital to obtain images that can be compared across various institutions.

A standard procedure includes the stabilization of the examination room temperature and humidity. The room should be within a stable range of $18^{\circ} \mathrm{C}$ to $20^{\circ} \mathrm{C}$; with maximum fluctuations of $1^{\circ} \mathrm{C}$ during, and an hour up to the examination [45], [46]. To maintain this temperature other sources of heat like sunshine and electronics should be eliminated from the room; this is done to reduce thermal artifacts [46]. The patient should be removed from drafts caused by air conditioning; this is done to reduce the physiological stress (shaking) that may negatively impact the procedure. There are two approaches that are practiced in preparing the patient for the procedure. In the first, patients are imaged $10-20$ minutes after they have undressed to cool the skin and allow deeper heat to surface. The second is to image the patient after a thermal stress 
test; exposed to a hot or cold stimuli [19]. The school of thought here is that cooling, or warming the skin will emphasize underlying hot and/or cold spots. The size of the image is dependent on the distance of the camera to the body, and the focal length of the camera [47]. The lens is usually of a fixed length, so it is important to keep the camera at a constant distance from the patients in order to reproduce this field of view (FOV). A standard view for each region of the body has been recommended, as in radiography, and includes: anterior, posterior, lateral and angled views [45], [47]. It is also recommended to establish a constant position for the patient, be it standing, sitting, or lying down. However, images of a specific body region captured with the patient in different positions cannot be compared [45].

IR camera technology is rapidly evolving, non-invasive, non-contact, low cost and a wide range of applications have been used to detect thermal abnormalities in a host of medical conditions. These applications include neurology [14], vascular disorders [15], breast cancer detection [16], [17] rheumatic diseases [18], [19]and has been strongly encouraged for neonatology [20]. Although reported results of sensitivity (proportion of true positives) and specificity (proportion of true negatives) vary according to the application, this may be attributed to the subjectivity introduced in the visual examination of thermograms. 


\section{Chapter 3: Literature Review}

This section aims to provide the necessary framework to understand the rationale behind the assessment of the inflammation associated with Necrotizing Enterocolitis (NEC) through medical thermography. It will provide an in-depth discussion on the various techniques adopted by other investigators in the quantification of thermograms, and how thermal abnormalities have been identified.

\subsection{STATE OF THE ART}

\subsubsection{GENERAL CONSIDERATIONS}

Abnormal thermal behaviour of skin can appear primarily as abnormal spatial distribution of temperature over the skin surface, and/or as abnormal dynamic behaviour (i.e. cooling, warming, or periodic fluctuations of temperature of a subarea of skin) [13]. Healthy subjects have skin temperature distributions that exhibit contralateral symmetry. An asymmetrical thermal distribution above a certain level or the presence of hot and/or cold spot(s) is an indicator of abnormality [12]. In the time domain, healthy skin should exhibit a uniform rate of temperature change over the surface of skin during cooling and warming [13]. When thermal equilibrium between the environment and the body has been reached, the rate of temperature change would approximate zero. Accordingly, non-uniform rates of temperature change of skin over time are also indicative of thermal abnormalities.

A vast portion of literature concerning medical thermography is heavily reliant on qualitative interpretation of thermograms. This relies on the ability to visually 
detect abnormal thermal variations of the skin temperatures based on the assessment of pseudo-coloured or grayscale thermograms. This task is difficult even for a trained thermographer and introduces a fair amount of subjectivity; findings are based on the ability to distinguish between the small or large variations of the intensity levels used to represent the temperature distribution of the thermogram. Pseudo-coloured thermograms used to map temperatures have also been criticised due to the psychological effect of colours on the perception of the observer, which may skew findings [36].

Quantitative analysis of thermal images has been attempted by some researchers to reduce the subjectivity introduced with visual assessments. Earlier studies evaluated the degree of thermal asymmetry observed within a healthy population; Uematsu et al. studied the degree of thermal asymmetry by observing a sample size of 90 healthy individuals between the ages of $19-59$. The temperature difference was computed for 40 matched regions over the entire body [12], [48]. Reproduced over a 5 year period, it was found that the temperature difference of contralateral regions (excluding extremities) was relatively small, generally less than $0.5^{\circ} \mathrm{C}$. The findings were also confirmed by Niu et al. who performed a similar study, using infrared images of 57 healthy volunteers, where the skin surface was divided into 25 regions. Average temperatures of the left and right regions were compared. They also noted variations in temperature differentials based on the region of examination; again the differences were no more than $0.5^{\circ} \mathrm{C}$ [49]. 


\subsubsection{STATISTICAL ANALYSIS}

In order to perform a statistical assessment of the temperature distribution for specific regions of skin, termed regions of interest (ROIs), these areas must be enclosed. The ROIs must be selected from thermograms in an anatomically precise manner, so that results of thermal analysis accurately represent the physiological process within the area of concern [50]. The ultimate goal was to segment the thermograms into smaller ROIs in order to extract and analyze relevant information. Montoro and Anbar suggest the use of anatomical landmarks to ensure precise ROI selection; the authors expose pitfalls of simple techniques that compute only the mean and standard deviation of ROIs of random sizes and shapes [51].

Lipari and Head selected ROIs that enclosed the left and right breasts based on anatomical landmarks (chin and nipple); each breast was further segmented into four smaller ROIs (quadrants). The analysis of quadrants was established to account for the expected mean temperatures of different regions of the breast (top and bottom), further aiding the identification of abnormalities [52], [53]. To quantify the degree of thermal symmetry, the authors compared statistical parameters between the whole breasts and the sum of differences for each quadrant. These parameters included the mean, standard deviation, median, maximum, and minimum temperatures. With these parameters the authors developed three modes of analysis: comparison of complete breast areas, comparison of quadrants, and hot spot analysis. The authors state that their findings were similar to those obtained through the visual assessment of a trained thermographer. 
Frize et al. improved upon the methods implemented by Lipari and Head on breast thermography. The authors found a correlation between the results of thermal analysis and clinical outcomes [54]. In a following paper [17], the authors stated that only the third method reported by Lipari et al. provided reliable results using a slightly larger population [17]. A higher classification rate was obtained by increasing the threshold used to discriminate between normal and abnormal breast thermograms.

Common statistical parameters computed for each ROI include: the mean, the median, the mode, the maximum, the minimum, the standard deviation and the skewness of the temperature distribution. A histogram depicts how many pixels have a certain intensity value within a discrete range of intensities. Presenting important statistical information about the temperature distribution of the image or ROIs and simplifies the identification of hot and/or cold spots to the determination of thresholds [50].

Mabuchi et al. implemented an image processing program that evaluated the difference in temperature distribution of the affected side and the contralateral healthy side. Once divided into the affected and normal parts, each part was further divided into symmetrically positioned trapezoid and triangle pairs. Temperature differences of symmetrical regions were then computed on a pixel-by-pixel basis. Investigators noted that this program was limited to evaluate pathologies of the breast, thorax and shanks [55]. 
Vavilov et al. measured thermal symmetry by comparing temperatures of the left and right breasts, the front and back of the thorax, and the anterior and posterior ankles of healthy individuals. To quantify thermal symmetry, contralateral ROIs were manually selected by the operator with small rectangles. The signal-to-noise ratio, Z-criterion and D-criterion (Kolmogoroff-Smirnoff's statistics) was then computed. The authors concluded that appropriate thresholds selected for each statistical parameter computed allowed successful differentiation between healthy and pathological cases. In addition, it was noted that different parts of the body have varied degrees of thermal asymmetry, which highlights the need for more rigid test procedures specific to the area of investigation [56].

Jakubowska et al. applied first order and second order statistical parameters to discriminate between healthy breasts and those with malignant tumours. The authors computed histogram based first order statistics and compared the absolute difference between the left and right breasts. These statistics included the mean, standard deviation, variance, skewness and kurtosis. Second order thermal signatures were obtained by computing the mean and variance of the grey-level cooccurrence matrix (GLCM) computed for each thermal image. Only horizontal and vertical directions were considered and a distance of 1 was used. The authors concluded that left to right differences in skewness was the most promising for use in classification of images. Absolute differences of second order statistical parameters, in particular the variance was able to successfully separate the majority of the healthy breasts from those with malignant tumours [57]. 


\subsubsection{SPATIAL METHODS}

Traditional spatial methods require that trained thermographers perform a visual assessment of thermograms in order to identify hot and cold spots in a qualitative manner. This is typically done by examining the entire image or comparing temperatures of contralateral symmetric regions. In some studies, images are collected after a thermal stress test is performed to identify areas with abnormal cooling or warming [41].

In an effort to quantify findings, some investigators have adopted the use of scoring indexes. Head et al. used an IR scoring method that ranged from 0 to 8 by the summation of individual metrics of abnormality in breast thermograms, i.e. size of hot spots or the position of heat patterns [58]. In a subsequent paper, an alternative scoring index ranging from 0 to 4 was used; the breasts were divided into four quadrants and the differences in mean temperatures of symmetrical regions were determined; a score of 0.5 or 1.0 was assigned if the difference was above $0.5^{\circ} \mathrm{C}$ or $1^{\circ} \mathrm{C}[16]$.

Keyserlingk et al. investigated the use of an IR grading system to aid the interpretation of images based on both quantitative and qualitative findings for early detection of breast cancer [59], [60]. This method was reliant on relevant clinical information such as the known causes of thermal abnormalities. Also the temperature difference between the left and right breasts were computed and compared to those values previously obtained. Grades were assigned based on the significance of the thermal sign and the associated pathology. 
Collins et al. used a thermographic index to quantify the degree of inflammation present in knee joints of patients with rheumatoid arthritis (RA) over the course of a steroid treatment [61]. The thermographic index was defined as:

$$
\boldsymbol{T I}=\frac{\sum(\Delta t \times \boldsymbol{a})}{\boldsymbol{A}}
$$

where, $\Delta t$ was the difference in the mean temperature of each measured isotherm $\left(0.5^{\circ} \mathrm{C}\right.$ intervals $)$ and the baseline temperature of the ROI $\left(26^{\circ} \mathrm{C}\right), a$ is the area of each of the isotherms and $A$ is the total area of the ROI. In a follow up study, the authors used this thermal index to evaluate the effects of non-steroid based treatments for RA patients, on the knees, hands, and feet [62]. They concluded that this technique was suitable for both short-term and long-term assessment of antiinflammatory treatment of RA patients.

Montoro and Anbar recommended the use of temperature profile lines that displayed temperature as a function of distance to obtain a more objective criterion [51]. They supported this claim by arguing that since temperature and temperature gradient profiles are not quantized like isotherms, the information content is not reduced; this provides a better diagnostic value. In order to compare findings of thermograms taken at different times or of different subjects, temperature profiles must be drawn between anatomically defined points. This methodology provides valuable information on the spatial distribution of temperature, which accentuates the presence of hot and/or cold spots, in addition to evaluating the symmetry of each feature. To evaluate the rate of temperature change, computing the derivative of the temperature profile with respect to distance was also recommended, 
especially to detect fast changes in temperature (e.g. subcutaneous blood vessels or capillaries) [51].

\subsubsection{TEMPORAL ANALYSIS}

Classical thermal imaging derives information based on the spatial distribution of temperatures within the area of interest, whereas dynamic (temporal) analysis derives information based on the change of temperature. Since skin temperature is controlled by the mechanism of thermoregulation and is a direct result of the subcutaneous circulation or disturbances to blood flow in underlying capillaries, it is important to also quantify the time-varying processes of changes in temperature distribution. However, this may not improve the spatial detection of hot and/or cold spots, but may offer valuable temporal and/or frequency information; by the identification of areas with abnormal cooling, warming, or thermal oscillations [51], [63].

To emphasize the temporal evolution of thermal patterns prior to imaging, subjects undergo a thermal stress test where they are exposed to a cold or hot stimuli so that warming or cooling can be observed. Many methods have been investigated to analyze the change and final state of temperature patterns in response to thermal stimuli; some examples are summarized below.

Dynamic temperature changes can be easily obtained by performing a simple subtraction of sequential thermograms prior to and after a thermal stress or acclimatization period [55], [64].

Ring [19] adopted cold stress testing as part of the imaging procedure for patients with Raynaud's phenomenon. The mean temperature differences between fingers 
and the back of the hand were compared before and after the stress test. This assessment provided an objective way to discriminate between normal and abnormal patients by quantifying abnormalities during thermal recovery. To describe the temperature gradient, a simple index was derived. This index also proved useful in the identification of patients with Reflex Sympathetic Dystrophy (RSD). From these studies it was possible to determine that an injured hand does not have a normal recovery from thermal stress. Merla et al., in a series of papers, confirmed these findings using a mild cold stress test; here the authors were able to differentiate between healthy patients from those suffering with the primary or secondary form of Raynaud's phenomenon [15], [65], [66].

Fujiwara et al. [67] studied the recovery rate of skin temperature after emersion in a cold water stress test in order to differentiate between healthy patients and those with type 2 diabetes. The authors found reduced thermal recovery rates in patients with diabetes. In a similar study[68], Zotter et al. studied the rewarming index of the lower legs of patients with type 1 diabetes after a cold stress test, by comparison of seven different sites on the leg. The authors found that the rewarming rate of those with diabetes was lower than that of the healthy population [68].

Ohashi and Uchida attempted to increase the sensitivity of breast thermography. They proposed a $\mu$-value image processing system, based on the premise that the thermal recovery of skin after a cold stress test can be modeled by a monoexponential function $\mu$ that varies with time [69],[70]. The $\mu$-values for each pixel were computed for sequential themograms and reconstructed to form $\mu$ thermograms. The intended purpose of the $\mu$-thermogram was to aid in the 
identification of pathophysiological abnormalities that manifest as thermal abnormalities.

Human skin plays a vital role in thermoregulation under the control of the autonomic nervous system. Skin temperatures oscillate around a certain set point temperature, which may vary. Since, the regulatory process is time varying in nature, it is appropriate to analyze thermograms in the frequency domain, which may yield information about pathological disorders [63], [71].

Montoro and Anbar calculated the Fast Fourier Transform (FFT) of time profiles, defined as the temperature of one pixel, or averaged over a given area as a function of time, from square ROIs covering the back; and determined the frequency characteristic of temperature oscillations after the removal of slow trends. The authors criticized static thermography by arguing that temperature asymmetries of less than $0.1^{\circ} \mathrm{C}$ should not be deemed significant, as the fluctuations of skin temperature in a short period of time can be at this level or higher [72].

Another method of performing temporal analysis of thermal images is to apply the Karhunen-Loève transform (KLT). KLT is an orthogonal transformation that achieves optimal results for the reduction of linear data. When used to analyze sequential thermograms, the number of images is reduced to a certain number of Eigen-images with the associated weights and times. Unser et al. claimed that these Eigen-images contained the majority of relevant spatial and dynamic information; providing a simple effective method to detect thermal abnormalities [73]. The authors used KLT expansions based on spatial and temporal models allowing an interpretation of results that favours either static or temporal components. In this 
study, standardization of the data was done with respect to the average image in order to distinguish between static and dynamic components of the images [73].

\subsubsection{IMAGE PROCESSING TECHNIQUES}

In current literature, new developments have emerged in the processing of thermal images to advance automation and improve the detection of thermal abnormalities. In most studies, the first step is to perform image enhancement to emphasize the texture and reduce unwanted influences. Typically this includes: noise reduction, background removal and contrast enhancement. Commonly followed by the segmentation of ROIs, then relevant features are extracted for interpretation or classification [64].

Pre-processing of thermal images has been investigated by many researchers; it is necessitated by the inherent level of noise present in all electronic devices. The presence of noise may create a pseudo texture that could lead to an erroneous computation of features if this texture dominates [50], [74]. Similarly, the lack of homogeneity in the background intensities may skew intensity-based techniques such as thresholding for segmentation. Many noise reduction techniques are available, but an optimal method is typically selected based on experimental findings [75]. The selected method must remove noise while preserving the edges and the resolution of the image. The "optimal" filter can be selected based on mean square error (MSE) values [75]. A larger size of filter produces better accuracy; however, this will also worsen the blurring of the image.

Herry proposed an automatic method for the assessment of pain in medical infrared images. A novel Poisson filter in the Wavelet domain was implemented to reduce 
noise. Noise was modelled as a Poisson distribution with white Gaussian noise. The author concluded that images filtered using this novel method was more easily processed in subsequent steps. Background removal was performed using a histogram based technique to establish a threshold. Then morphological and logical operations were used to fill in holes that may have resulted from the use of aggressive thresholds. An automatic approach to divide the body into rectangular grids was implemented, based on the identification of the vertical line of symmetry and anatomical reference points. ROIs were classified as being thermally normal or abnormal based on comparisons performed between the histograms of other ROIs. The Euclidean distance was determined to be the best statistical method of those measured in the differentiation of the control population from the pain patients [41].

Herry's doctoral thesis developed a framework for the automatic segmentation of ROIs and identification of anatomical regions of interest. Three approaches for segmentation were explored. The first method named cued morphological processing of edge maps produced results that were superior to the best methods available in 2008. To limit the extent of morphological processing of edge maps minimal amount of prior information known about the ROI was used [76]. The second technique attempted to classify edge components using Support Vector Machines or Neural Networks. This resulted in the generation of partial contours that in combination with other methods recovered missing information. The last method was a fusion of the last two, optimal thresholding and Fuzzy C-Means clustering. In comparison to other fusion methods available at the time, the method 
attempted here proved to be better [77]. The identification of anatomical ROIs using anatomical landmarks derived from the contours of the body was also presented. The approach performed curvature analysis for region contours, the distance between contours of symmetric regions, and region specific constraints. Localised clusters of landmarks were created that returned the location of key anatomical landmarks of the region contours [77], [78].

Wiecek et al. developed an image processing tool that found a visible connection between thermal and radiological images capturing the inflammation caused by pneumonia. This interactive tool was based mainly on statistical data processing. Here, the user controlled the margins of the histogram and set the threshold level for segmentation. The first step of this technique performed contrast enhancement using histogram equalization. In the second, histogram stretching and segmentation was performed. The ultimate goal was to select optimal values that may highlight the border of areas with inflammation [50], [79].

Snyder et al. approached the task of noise reduction by developing an algorithm that magnified the infrared images by a factor of two while simultaneously preserving the fine details, like the sharpness of the edges. An optimal interpolation algorithm was used based on the philosophy of maximum a-posteriori probability (MAP), which estimated new data based on knowledge of the current image. The authors formulated an objective function to assess the quality of the estimation performed, which was optimized using Mean-Field Annealing (MFA). In addition, white Gaussian noise with a mean of zero was introduced into the images. The authors 
applied this approach to breast thermograms and found that this image restoration method performed even better with increasing the level of noise [80].

Qi et al. proposed an automatic approach to the detection of thermal asymmetry for the detection of breast cancer. A Canny edge detector was used on the original image to obtain the edges. The Hough transform was then applied to the edge image to detect the boundaries of the body and the curvature of the breasts; segmentation was performed in accordance with the intersection of specific boundaries and curvatures. In order to determine the degree of thermal asymmetry in breasts, the smoothed histograms were computed and compared [81].

Bao-ping and Ma proposed a similar approach to perform the automatic thermal symmetry analysis of breast infrared images. Canny edge detection and Hough transform were performed to segment the breasts. A k-means clustering algorithm was used to make an image that would be used for classification. The algorithm initially assigned each pixel to a random cluster; the average for each cluster was then computed. The algorithm iteratively computed the distance of each pixel to the cluster centres and classified each pixel to the cluster of minimal distance; the average of each cluster was then recomputed. Once all pixels have been assigned to a cluster and their averages and centres were known, asymmetric abnormalities were easily identified [82]. 


\subsection{DISCUSSION}

Medical thermography has been a major research interest for the past few decades. This was made possible by the advancement of technology, which increased the spatial and temporal resolution of infrared cameras [12], [13]. The identification of asymmetric temperature distributions of the body, and hot and/or cold spots, suggests underlying pathology [12], [13]. Visual examination of thermal images is very difficult, even for an experienced thermographer. For this reason, quantitative methods are desirable to reduce the subjectivity introduced during visual examination [16], [36]. Standards for the acquisition of images have been well established [47], [83]. The importance of proper region of interest selection has been repeatedly stressed, which may increase the processing time if performed manually. For this reason, automated analysis of IR image processing has also been a major research focus, and many successful approaches have been reported [77], [81], [82]. Thermal symmetry has been measured by comparing simple statistics extracted from symmetric regions of the body [36], [52], [55], [57]. The extraction of second ordered statistical features has been explored to a lesser extent [57]. Segmentation of the abdominal region from the rest of the body has not been researched.

This work evaluated the potential of abdominal IR thermal imaging as a tool in the identification of inflammation associated with NEC. The potential of using IR imaging in the identification of NEC has also been postulated by Rice et al. [21]. These investigators aimed to examine the feasibility of thermography in the assessment of abdominal skin temperature of extremely low birth weight infants 
(ELBW). They compared abdominal and thoracic skin temperatures that were enclosed by oval shaped ROIs. A secondary goal was to explore the relationship between abdominal skin temperature and NEC. They enrolled 13 infants that were of ELBW (extremely low birth weight) and were born premature (25-29 weeks), 10 of these participants underwent radiographic and thermal imaging. Serial thermal images were captured while the infants were in the incubator. The authors concluded that thermal imaging was feasible to be performed with the infant population, and that infants with radiographic evidence of NEC had lower abdominal skin temperatures in comparison to the healthy population. They attributed these lowered temperatures to the likely presence of advanced intestinal ischemia (reduced blood supply to tissues). However, they state that this work should be viewed as a preliminary phase to assess safety and feasibility of thermography [21].

In this work a manual method of abdominal region (ROI) selection was adopted, which allowed for anatomical accuracy. The remainder of this system was fully automated. Using first order and second ordered statistical features the degree of abdominal thermal symmetry was assessed. In addition, appropriate methods of image pre-processing and enhancement were implemented. Ultimately, features extracted from the abdominal thermal sequences obtained from NEC and normal infants were compared. The aim was to assess the degree of thermal symmetry of infants with and without NEC. 


\section{Chapter 4: Identification of Inflammation Associated}

\section{with NEC Through Infrared Imaging}

This section discusses in detail the problem of assessing abdominal infrared images for the detection of thermal abnormalities, which may indicate the presence of inflammation associated with NEC. The first section presents major components of medical IR imaging systems commonly used, and relevant issues expected with the analysis of infrared images. This section proposes a solution that aims to address these concerns. Section 4.2 focuses on the protocol and acquisition of abdominal IR images, which includes the normal and NEC population. The last section discusses the image processing and analysis methods adopted.

\subsection{METHODOLOGY For ANALYSIS OF ABDOMINAL INFRARED THERMAL IMAGES}

This thesis addresses the problem described by providing a solution to two major problems.

The first problem was to attempt the quantitative analysis of static abdominal infrared images using computerised techniques. Thermal symmetry analysis was performed by the comparison of first and second order thermal signatures extracted from contralateral symmetric regions. A statistical comparison of each feature was then performed to compare the distribution of values obtained for the normal and NEC groups. 
The next step was to develop an image processing procedure that was suitable for the pre-processing, enhancement and segmentation of medical infrared images. Automation of procedures was implemented where possible.

Image pre-processing techniques specific to this problem were developed. This included the rotation and normalization of images, which was necessitated by the need to ensure accuracy in the comparison of temperature distributions of contralateral symmetric regions.

Incorporation of noise reduction and background removal techniques is usually disregarded by most investigators, although this may have serious consequences in more advanced image processing techniques and intensity-based operations.

As a result, the second problem tackled was the development of image enhancement techniques that are appropriate for use in medical thermography. The goal was to improve the interpretability or perception of images and unveil details that were previously hidden while maintaining important information. These automatic procedures included: noise reduction, background removal, and contrast enhancement whose goals are summarized below.

1. Noise reduction aimed to reduce acquisition noise that is inherent to all electronic devices, while preserving important information that contains edges and fine details.

2. Background removal was incorporated to segment the body from the rest of the image, ultimately to minimize its influence on the visual examination of thermal images. 
3. Exploration of enhancement techniques suitable for use in medical thermal imaging to reveal hidden details of images.

Following this the identification of regions of interest was performed. This step was vital in the analysis of temperature distributions of specific areas of interest. Although, solutions have been proposed in some of the literature available, precise segmentation of the abdomen has yet to be discussed. In this work manual selection of the ROI was adopted.

In addition, all these components were integrated into a full system. The objective was to reduce the processing time, facilitate easy reproduction of results and save important information (data structures, images and results).

\subsection{Data Collection}

\subsubsection{EQUIPMENT}

A sequence of thermal images of the abdomen were captured using a longwavelength IR camera (uncooled microbolometer focal plane array, $320 \times 240$ pixels, thermal and spatial sensitivity of $0.05^{\circ} \mathrm{C}\left(\right.$ at $\left.30^{\circ} \mathrm{C}\right)$ and $1.3 \mathrm{mrad}$, respectively) [84]. The recordings were then transferred to a laptop for further processing.

\subsubsection{PATIENT RECRUITMENT}

This study aims to assess the potential of infrared thermography in the detection of infants with "classical" NEC. Under this definition, subject recruitment was limited to those babies who were less than $32+6$ weeks gestational age and had a VLBW (less than 1500 g). Infants were recruited at the Children's Hospital of Eastern Ontario (CHEO) and at the Ottawa General Hospital (OGH) between Dec. 2006 and Jan. 2010, following the approval from the Ethics Review Board of both hospitals 
and informed consent from the parents [84]. These documents can be found in Appendix A Section A.1 Research Ethics Proposal and Section A.2 CHEO Parent Information Sheet.

The healthy group was selected from amongst those infants who were born prematurely and were of VLBW, but who showed no clinical, radiographic, or pathological signs associated with NEC, or had no other condition that altered thermoregulation. The NEC group was composed of infants who exhibited clinical signs associated with NEC, and had radiographic evidence of Bell's stage 2 or higher. Infants who had intra-abdominal congenital anomalies, active sepsis, or umbilical or abdominal dressings occluding the imaging area were excluded from the study. In total 48 healthy babies $(21 \mathrm{M} / 27 \mathrm{~F}$, gestational age $28.3 \pm 2.4$ weeks, birth weight $1127 \pm 497 \mathrm{~g})$ and 11 NEC babies $(5 \mathrm{M} / 6 \mathrm{~F}$, gestational age $26.7 \pm 1.8$ weeks, birth weight $969 \pm 430 \mathrm{~g}$ ) were enrolled [84].

\subsubsection{IMAGING PROTOCOL}

Before sequential infrared images were captured, the babies were removed from their thermally controlled environment (incubator) of approximately $30^{\circ} \mathrm{C}$ for approximately 45-60 seconds [84]. Typically medical thermography requires an acclimatization period of $10-20$ minutes to allow skin temperature to stabilize, but the thermoregulation of infants is not fully developed and they require a high temperature environment. However, removal of infants from their warmer incubator for 30 to 60 seconds prior to imaging acts as a cold stress test, allowing for the measurement of abdominal skin cooling. A sequence of thermal images was acquired at a rate of 30 frames per seconds for 60 seconds. The baby was imaged in 
the supine position with the camera placed approximately $60 \mathrm{~cm}$ from the abdomen in a downwards position, as illustrated in Figure 4-1. Recordings were then transferred onto a laptop for analysis at a later time. Images were collected prior to the commencement of this thesis by Herry et al. [84].

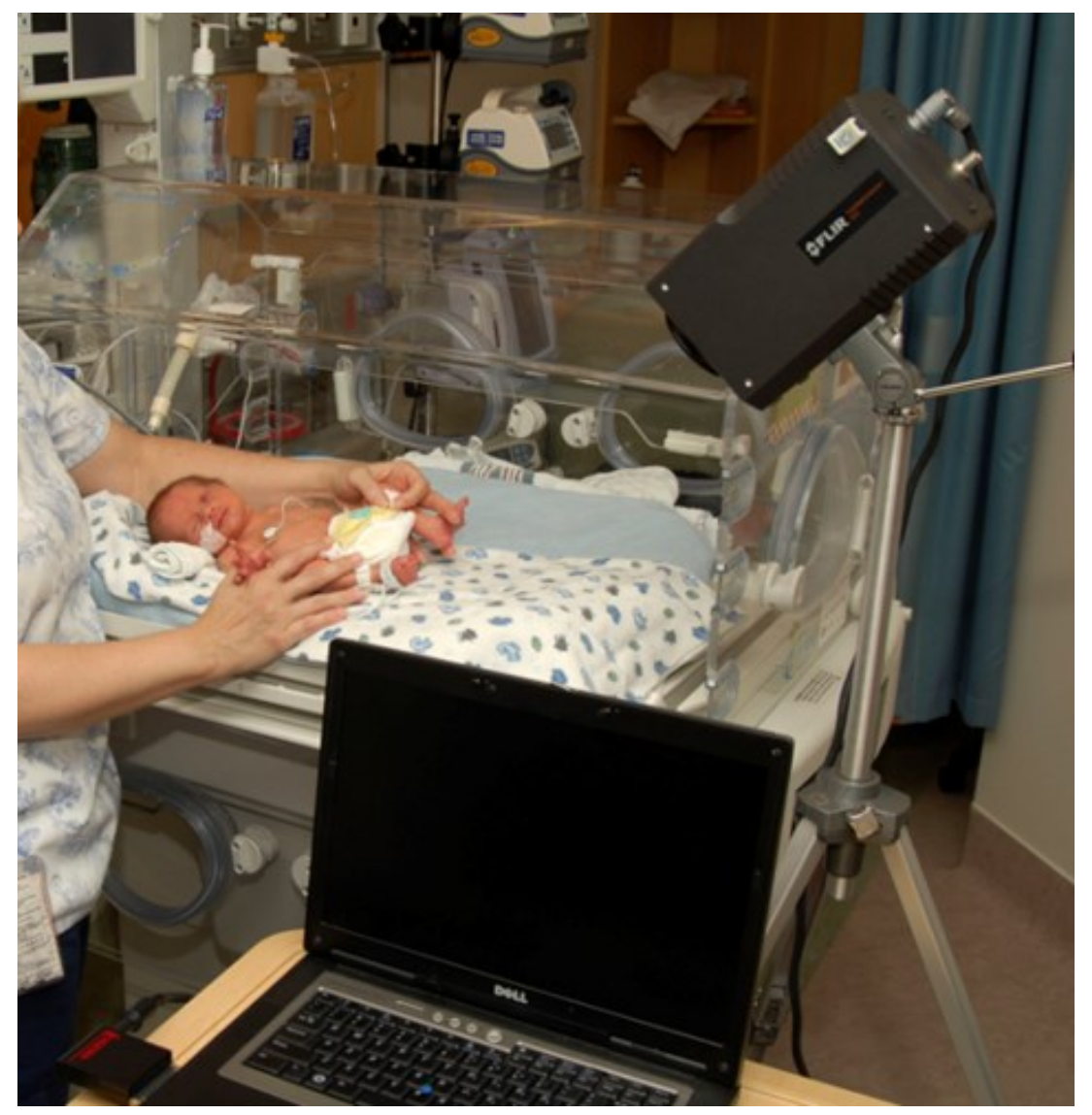

Figure 4-1: Example of how serial thermal images of the abdomen were captured.

To measure if there was a significant drop in body temperature during the procedure, axial and rectal temperatures were taken before and after the procedure. In addition, the nurses assessed each baby's level of pain during the imaging procedure using the Premature Infant Pain Profile (PIPP) Score [84]. 


\subsubsection{IMAGE SELECTION}

Prior to processing abdominal thermal images it was necessary to develop a procedure for selecting data in order to obtain legitimate results. The following images were excluded:

- Sequences that involved too much movement, where the infant appeared to be aggressively trashing. These recording had a great deal of movement artefacts that resulted in blurring. A total of 10 sequences were removed.

- Images that did not capture the full abdomen in an almost perfectly supine position. Meaning that the abdomen was captured at an angle that was more than $\pi / 4$ [12], [35]. A total of 13 recordings were removed.

- Images where the abdomen was momentarily occluded. In some instances the umbilical stump was very large and covered a significant portion of the abdomen. A total of 7 recordings were removed.

After removing the poor recordings, the final data set used in this study consisted of 20 normal infants and 9 infants diagnosed with NEC. Herry et al. [84] captured sequential infrared images for 60 seconds and assessed the abdominal cooling rate of infants. The difference in the average abdominal temperature over 60 seconds was small [84]. For this reason, it was decided that averaging of temperature over 20 frames would be sufficient for the analysis conducted in this study. This work aimed to assess the degree of thermal symmetry. For each infant the first 20 to 60 suitable frames captured during the first 60 seconds of the imaging were used. 


\subsection{Thermal Image Processing Techniques}

This section discusses in detail the methods used in the development of an image processing approach for the analysis of abdominal infrared thermal images for the purpose of identifying inflammation associated with NEC. The proposed system described in Figure 4-2 was divided into four stages: image pre-processing, image enhancement, ROI segmentation, and image analysis.

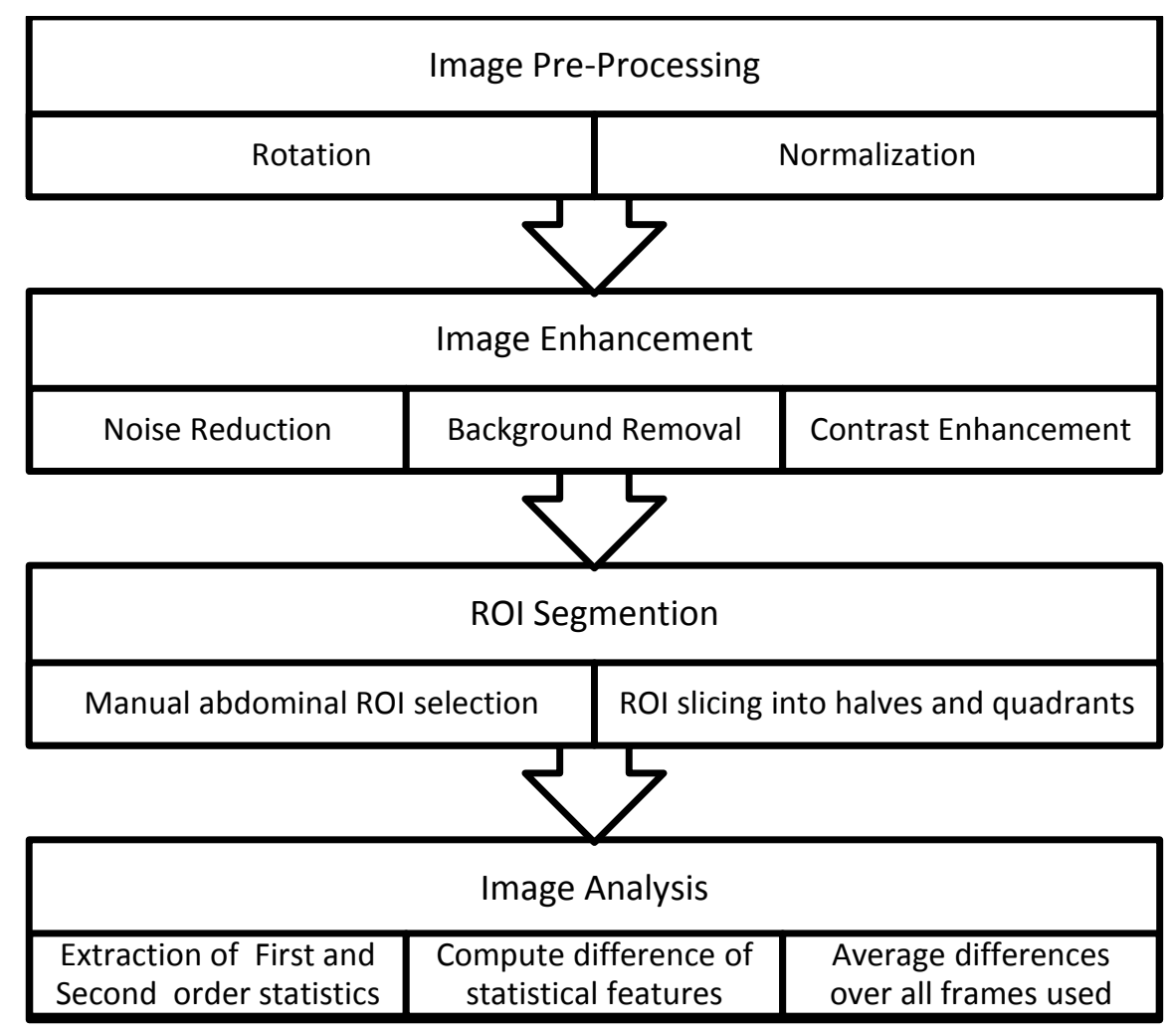

Figure 4-2: This flow diagram depicts the four steps used to process, enhance, segment and analyze thermal images. ROIs available were the: whole, left, right, upper, lower, right upper quadrant (RUQ), right lower quadrant (RLQ), left upper quadrant (LUQ), and left lower quadrant (LLQ). First order and second order statistical features were computed for each ROI, and the differences between the upper-to-lower, left-to-right, and sum of quadrants-to-whole (QTW) were computed and averaged over all useable frames.

\subsubsection{IMAGE PRE-PROCESSING}

The goal of pre-processing was to prepare the thermograms for further enhancement and analysis. In order to standardize the colour scale of the image, it 
was normalized to a grayscale [45], [47]. Most frames selected for analysis were captured with the infant in the correct position, but in some instances the body was slanted and these images required rotation.

\section{Normalization}

The abdominal infrared images used in this study were first exported into MATLAB as an array; here each pixel represented a temperature value (in Kelvin). This array was converted into degrees Celsius $\left({ }^{\circ} \mathrm{C}\right)$ by performing the simple operation in the equation below.

$$
\text { ImageArray }_{\text {Celcius }}=\text { ImageArray }_{\text {Kelvin }}-237.15
$$

With the available temperature information and use of a linear mapping function, these arrays or intensity image (I) were transformed into an 8-bit grayscale image according to equation 4.2 .

$$
\boldsymbol{I}_{\boldsymbol{n}}=(\boldsymbol{I}-\boldsymbol{M i n i m u m}) \times\left(\frac{\text { NewMaximum-NewMinimum }}{\text { Maximum-Minimum }}\right)+\text { NewMinimum }
$$

Here I represents the unprocessed thermal image from which the minimum and maximum values extracted. The newMaximum and newMinimum were defined as 255 and 0 to normalize the image to an 8-bit grayscale $\left(\boldsymbol{I}_{\boldsymbol{n}}\right)$. Normalization was not performed on original thermal images when first order thermal signatures were extracted, this will be further explained in Section 4.5 Image Analysis.

\section{Rotation}

It is often difficult to perform medical imaging of the infant population due to movement and the inability to follow a strict physical protocol. Although, thermal sequences with too much movement have already been removed from the data set, in some recordings the body was still not in the correct position. To ensure precise 
comparisons of temperature distributions of contralateral symmetric regions of the abdomen to be measured, the images must be rotated. A geometric transformation was performed which mapped the position of a pixel $\left(\mathrm{x}_{1}, \mathrm{y}_{1}\right)$ of the input image $(\mathrm{A})$ into a position $\left(\mathrm{x}_{2}, \mathrm{y}_{2}\right)$ in the output image (B) by rotating it by a user-specified angle $\theta$ about the centre pixel $\left(\mathrm{x}_{0}, \mathrm{y}_{0}\right)$. A positive angle $(\theta)$ resulted in the rotation of an image in the counter clockwise direction, while a negative angle rotated in the clockwise direction. The transformation of the pixel to a new location is described below.

$$
\begin{aligned}
& x_{2}=\cos (\theta) *\left(x_{1}-x_{0}\right)-\sin (\theta) *\left(y_{1}-y_{0}\right)+x_{0} \\
& y_{2}=\sin (\theta) *\left(x_{1}-x_{0}\right)-\cos (\theta) *\left(y_{1}-y_{0}\right)+y_{0}
\end{aligned}
$$

To compute the value of the pixel at this new position nearest neighbour interpolation was selected because maintenance of original values was imperative [85], [86]. This method computed the value of the new point based on the value of the old pixel coordinate that was nearest to the new pixel coordinate. No new values were computed by this algorithm, although some original pixel values were not used and others may have been duplicated; sharpness of the image will be reduced to some extent. The angle of rotation was no more than 15 degrees and was determined by trial and error. Here the investigator visually examined the images and estimated the angle until the best alignment was observed.

\subsubsection{IMAGE ENHANCEMENT}

Automatic enhancement of images is proposed as a three part process: noise reduction, background removal and contrast enhancement. Each step is discussed in 
detail to develop an understanding of the methods used and their parameters. The rationale behind their use will be explained.

\section{Noise Reduction Approaches}

The presence of noise is an inherent property of all electronic devices [87]. Typically, this noise is minimized to a marginal level, but is still introduced during image acquisition. Noise has the potential to introduce a pseudo texture to the image, which may confound metrics [88]. For this reason it is evident that noise reduction methods must be incorporated.

The principal operation of noise reduction methods is to determine if differences in pixel values of an image are real values; if found to be noise, these values are averaged to reduce their overall influence. Since the exact amount of noise content cannot be exactly quantified, a trade-off exists between noise reduction and the preservation of fine details such as edges. This work explores different edge preserving and neighbourhood methods.

In this study spatial filters denoted by $N \times N$ kernels were used to represent the shape and size of the neighbourhood. It is common practise to use spatial filters that are uneven in size to ensure that each pixel is located in the center of the neighbourhood [75]. As well, the larger the neighbourhood size the more noise is reduced, but the greater the extent of blurring results in the image. Normally $3 \times 3$, $5 \times 5$ and $7 \times 7$ sizes are selected for analysis, because anything above is sure to cause too much blurring and remove important content. As well, to preserve the brightness of the original image, it is necessary that the sum of all elements of the filter equate to 1 . 
Mean (or average) filtering is one of the simplest ways to smooth and reduce noise; each pixel is replaced with the mean intensity value of its neighbours [87]. In other words, the variations of intensities within neighbourhoods are smoothed and pixels that vary significantly are eliminated by smoothing. An example is the $3 \times 3$ kernel with even weighting below.

\begin{tabular}{|c|c|c|}
\hline$\frac{1}{9}$ & $\frac{1}{9}$ & $\frac{1}{9}$ \\
\hline$\frac{1}{9}$ & $\frac{1}{9}$ & $\frac{1}{9}$ \\
\hline$\frac{1}{9}$ & $\frac{1}{9}$ & $\frac{1}{9}$ \\
\hline
\end{tabular}

A median filter is a non-linear filter that is known to preserve the edges and fine details of an image while removing noise [87]. Given a $M \times N$ neighbourhood, this method orders the pixel intensity values and the median is selected as the output pixel; extreme values are not used in this computation [75]. The complexity of this method would be increased if even sized filters were used.

The Weiner filter is known to be one of the best ways to reconstruct a noisy image or signal. This adaptive technique is known to provide superior results in comparison to linear methods, attributed to the preservation of high frequency content that contains fine details. A Weiner filter is created for every pixel in the image using estimates of the mean $(\mu)$ and variance $\left(\sigma^{2}\right)$ of a local area, equations 44 to 4-5. Here, $\eta$ is the $M \times N$ neighbourhood with respect to pixel $a$. These estimates are used to create a pixel-wise Weiner filter (equation 4.6). In this equation, $v^{2}$ is the noise variance which is determined by averaging all of the 
estimated variances. The edges are preserved by this method, since when the variance is low, smoothing is high, and when the variance is high, smoothing is minimized. For this reason, it remains appropriate to use for noise reduction of medical images.

$$
\begin{aligned}
& \mu=\frac{1}{N M} \sum_{n_{1}, n_{2} \in €} a^{2}\left(n_{1}, n_{2}\right) \\
& \boldsymbol{\sigma}^{2}=\frac{1}{N M} \sum_{n_{1}, n_{2} \in €} \boldsymbol{a}^{2}\left(\boldsymbol{n}_{1}, \boldsymbol{n}_{2}\right)-\boldsymbol{\mu} \\
& \boldsymbol{b}\left(\boldsymbol{n}_{1}, \boldsymbol{n}_{2}\right)=\boldsymbol{\mu}+\frac{\boldsymbol{\sigma}^{2}-v^{2}}{\boldsymbol{\sigma}^{2}}\left(\boldsymbol{a}\left(\boldsymbol{n}_{1}, \boldsymbol{n}_{2}\right)-\boldsymbol{\mu}\right)
\end{aligned}
$$

The flow diagram below describes the simulation performed in Section 5.1.1 to determine the best filter from among those tested.

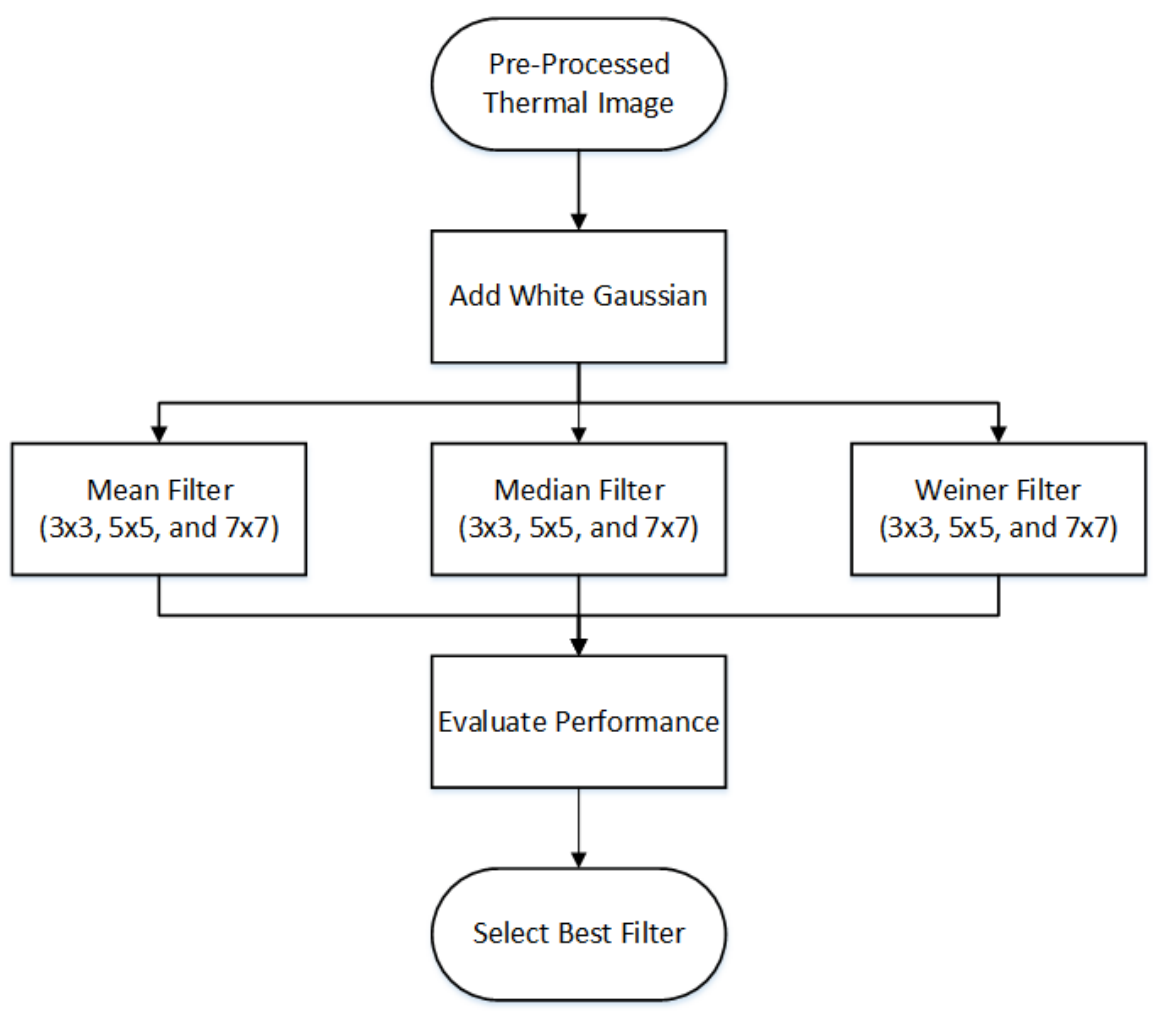

Figure 4-4: Block diagram depicting the simulation performed to determine which filter was the best. The mean, median, and Weiner filters of size $3 \times 3,5 \times 5$, and $7 \times 7$ were compared. The mean square error was computed to evaluate performance and the filter with the lowest value was selected. 
In a comparison of all the filters explored, the Wiener $(5 \times 5)$ filter was selected as the best filter. This filter was selected because it did not cause visible blurring and had the minimum mean square error (MSE) value of 5.22. This filter outperformed the best median and mean filters that had MSE values of 5.66 and 8.72. This is discussed in Section 5.1.1 Noise Reduction.

\section{Background Removal}

The purpose of background removal is to remove undesirable parts of the image, while maintaining important information. In this study we define the background as anything other than the region of interest (the body), including: clothing, wires, room, etc. When considering thermal imaging, the intensity of the body in comparison to the background of IR images is easy to separate, because more radiation is emitted by the skin and thus intensity values are also greater than that of the background. For this reason it is appropriate to use intensity-based segmentation algorithms to automatically define a grey level threshold that is able to separate the body and the background.

In this study, Otsu's algorithm was selected to perform automatic background removal [89]. This method was selected because it is simple, known to be stable and widely used in medical image processing. Segmentation is based on the computation of an optimal gray level threshold; the following discussion will show how optimization is achieved.

Given that an image has $L$ different grey levels, with $n_{i}$ representing the number of pixels of a given intensity $i$, and $N$ is the total number of pixels, the probability distribution $\left(p_{i}\right)$ is as defined in equation 4-7. A grey level threshold $k$ is used to 
segment the image into two classes $C_{O}$ and $C_{1}$ denoting the background and object of interest. Pixels with intensity values within the range of [1 k] are assigned to group $C_{O}$, and those between [k+1 L] to $C_{1}$. The probability of occurrence $\left(\omega_{O}\right.$ and $\left.\omega_{1}\right)$ and mean intensity values $\left(\mu_{O}\right.$ and $\left.\mu_{1}\right)$ of the respective groups are given in equations 48 and 4-9. The zeroth and first cumulative moments up to a $k$ th grey level is given in equations $4-10$ and 4-11. The total mean grey level for the image is as defined in equation 4-12.

$$
\begin{gathered}
p_{i}=\frac{n_{i}}{N} \text {, where } p_{i} \geq 0 \text { and } \sum_{i=1}^{i=L} p_{i}=1 \\
\omega_{O}=\sum_{i=1}^{k} p_{i} \text { and } \omega_{1}=\sum_{i=k+1}^{L} p_{i} \\
\mu_{O}=\sum_{i=1}^{k} i p_{i} \text { and } \mu_{o}=\sum_{i=k+1}^{L} i p_{i} \\
\omega(k)=\sum_{i=1}^{k} p(i) \\
\mu(k)=\sum_{i=1}^{k} i p(i)
\end{gathered}
$$

Using the zeroth and first cumulative moments along with the probability distribution defined in equations 4-7 to 4-9, the variances of the two classes are:

$$
\sigma_{o}{ }^{2}=\sum_{i=1}^{T} \frac{\left(i-\mu_{0}\right)^{2} p_{i}}{\omega_{o}} \text { and } \sigma_{1}{ }^{2}=\sum_{i=1}^{T} \frac{\left(i-\mu_{1}\right)^{2} p_{i}}{\omega_{1}}
$$

With the definitions of the class variances $\left(\sigma_{o}{ }^{2},{\sigma_{1}}^{2}\right)$, means $\left(\mu_{0}, \mu_{1}\right)$, frequencies $\left(\omega_{o}, \omega_{1}\right)$, the total mean level $\left(\mu_{T}\right)$ and the probability distribution $\left(p_{i}\right)$ given above, the within-class, between-class and total variance are defined as:

$$
\begin{array}{ll}
\sigma_{W}{ }^{2}=\omega_{o} \sigma_{o}{ }^{2}+\omega_{1} \sigma_{1}{ }^{2} & 4-14 \\
\sigma_{B}{ }^{2}=\omega_{o} \omega_{1}\left(\mu_{1}-\mu_{0}\right)^{2} & 4-15 \\
\sigma_{T}{ }^{2}=\sum_{i=0}^{i=L}\left(i-\mu_{T}\right)^{2} p_{i} & 4-16
\end{array}
$$


This technique defines the optimal threshold at a grey level $k^{*}$ as that which maximizes the criterion below, representing the between class variance.

$$
\eta=\sigma_{B}{ }^{2} / \sigma_{T}{ }^{2}
$$

Recall that infants are imaged while lying on sheets when the mattress is rolled out of the incubator. For this reason it may be difficult to fully segment the body, due to the transfer of heat from the body to contact surroundings. Although this study does not require segmentation to be perfect because manual ROI selection was used. Rather it is sufficient that the majority of the background was removed, to minimize its influence on the visual assessment of thermograms [45], [47].

\section{Contrast Enhancement}

Contrast enhancement methods are not designed to manipulate the structural information of the image, but rather to enhance certain characteristics by improving the image contrast [90]. An increase to the current level of contrast in an image results in the appearance of hidden details. Histogram equalization is a way of adjusting image intensities by enhancing the global contrast. The basic concept of this is to spread out cluttered frequencies over the histogram. The transformation of these intensities is described in equation 4-18 [75]. Here, $I_{k}$ is the intensity value of the enhanced image corresponding to the input intensity $r_{k}$, and $\mathrm{L}$ is the number of intensity levels of the image. The probability density function of a particular intensity is $p_{r}\left(r_{j}\right), n_{j}$ is the number of pixels at the intensity $j$, and $n$ is the total number of pixels. This method of transformation is said be prone to noise amplification, due to the fact that a number of pixels are within a certain intensity range [91]. 


$$
\begin{gathered}
I_{k}=T\left(r_{k}\right)=\sum_{j=0}^{k} p_{r}\left(r_{j}\right)=\sum_{j=0}^{k} \frac{n_{j}}{n} \\
0 \leq r_{k} \leq 1 \text { and } k \epsilon[0, \ldots, L-1]
\end{gathered}
$$

Contrast-Limited Adaptive Histogram Equalization (CLAHE) has been proven to be effective and widely used method of contrast enhancement for medical imaging, and is currently preferred for its usefulness in automated image processing [90], [92]. Unlike the conventional method of histogram equalization, this technique performs contrast enhancement over smaller regions of the image termed tiles. The intensity of the pixel is transformed to a value within the range of display that is proportional to the rank of the pixel intensity with respect to the local region [90], [92]. The difference between CLAHE and adaptive histogram equalization (AHE) is that there is a user specified maximum (clip level or clip limit) of the local histogram height. In other words, the histogram values are clipped beyond a specified limit and what is left over will be redistributed to other bins. This results in low contrast enhancement in homogeneous areas of the image, thereby preventing the over enhancement of noise caused by the enhancement of minute pixel variations, and minimizes the edge-shadowing effect [90]. The size of the neighbourhood and the clip level are necessary user-specified input parameters needed for CLAHE.

In this work the default 64 number of tiles $(8 \times 8)$ and a uniform distribution was used. The clip-limit values chosen for asymmetry analysis were 0.005 and 0.01 since very subtle changes to the images were noted and MSE values were minimal (54.3699 and 99.9086) [93]. As expected, the MSE values increased with the cliplimit. How this parameter was determined is discussed in Section 5.2.1 Contrast Enhancement 


\subsubsection{REgION OF INTEREST SELECTION}

A semi-automated approach to ROI selection was adopted, as proposed in literature [93], [94]. The whole abdominal and umbilicus regions are enclosed in oval shaped regions selected by the investigator, as shown in Figure 4-5. Since the umbilical region has a visibly different temperature it remains also excluded from the region of analysis. A centroid is calculated based on the umbilical ROI and used to segment the abdomen further into the: upper, lower, left right, right upper quadrant (RUQ), right lower quadrant (RLQ), left upper quadrant (LUQ), and the left lower quadrant (LLQ), see Figure 4-5. A black and white (BW) mask is created for each of these 8 regions, in addition to that of the whole abdominal ROI, created by subtracting the umbilical region from the whole abdomen. In subsequent images, the size and the pixel location of the whole abdominal ROI remain as selected previously but can be manipulated by simply moving it in the event that movement occurred.

The location of the whole ROI is selected with precision to ensure that the entire abdomen is enclosed. This is done by first selecting the umbilical region to provide some direction when selecting the whole abdomen. Great care is taken to not include the portions of the chest as the skin temperature here varies from that of the abdomen. 


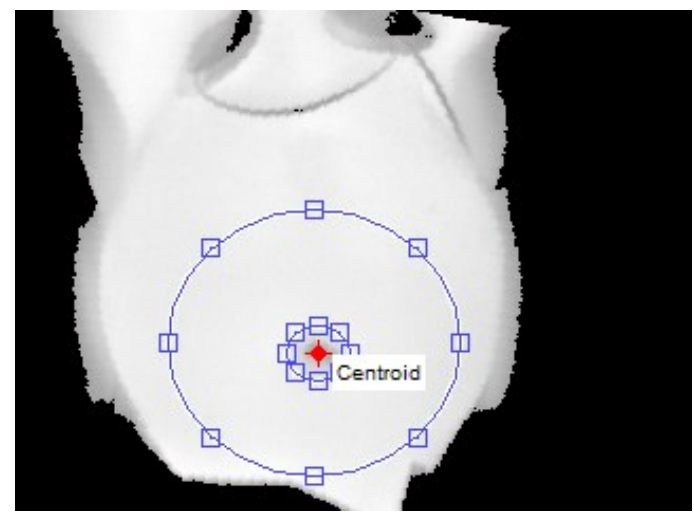

(a) Abdominal thermal image depciting the ROI selection process. Here the arms are raised above the head pointing north The wires around the NEC are connect to electrodes. The abdomen and umbilicus regions are enclosed by the large and small oval shapes. The centroid is determined based on the umbilicus ROI.
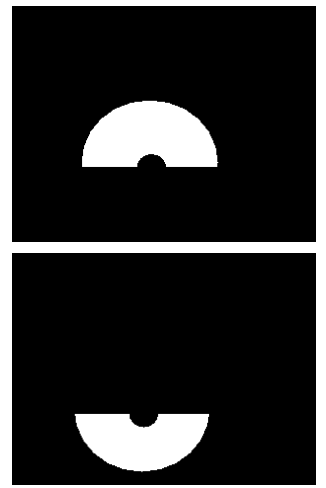

(C) Upper and Lower ROIs
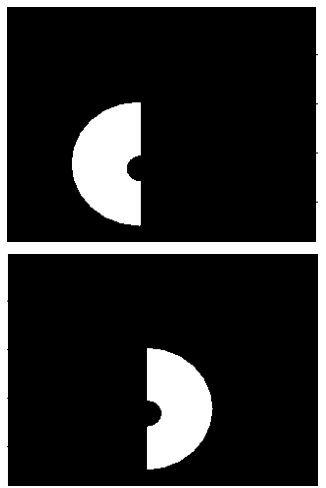

(d) Left and Right ROIs

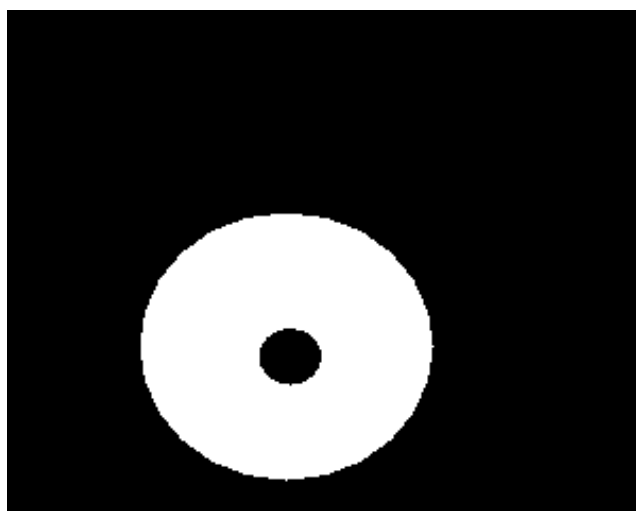

(b) The whole abdominal ROI, created by subtraction the umbilicus region from the abdominal ROI. This ROI is sliced into halves and quadrants depicted below based on the location of the centroid.
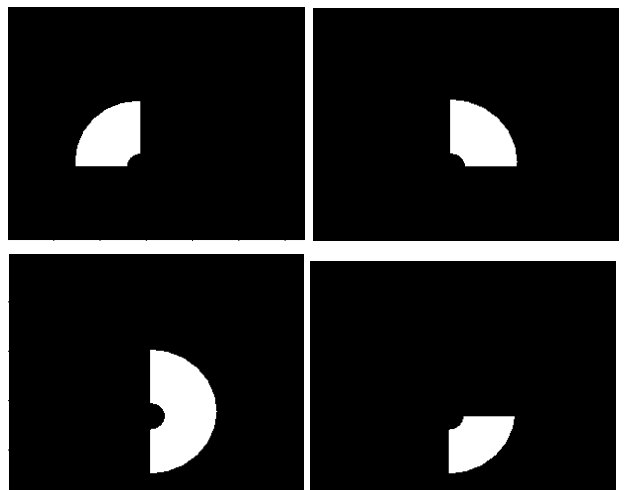

(e) Four quadrants (RUQ, RLQ), LUQ, and LLQ)

Figure 4-5: (a) Depicts the semi-automated ROI selection of the whole and umbilicus regions. The centroid is determined based on the umbilicus region (b) The whole abdominal ROI is sliced based on the location of the centroid to further segment the whole abdomen into halves (c-d)and quadrants (e).

\subsection{IMAGE ANALYSIS}

This work aims to perform thermal symmetry analysis of abdominal ROIs by comparison of statistical features which facilitates the objective assessment of the thermal distribution. In this study, statistical methods are used to analyze the spatial distribution of grayscale values. This is achieved through computation of statistical features for each point in the image or within a local region. The number of pixels used in a computation defines the order of the statistics: first-ordered (one pixel), second-ordered (two pixels), and high-ordered (three or more). 
First-ordered statistics measure the likelihood of a grey-level (or intensity) value occurring at any given location; these features can be computed using the histogram. Since these features depend solely on individual pixel values, they do not portray the relation or co-occurrence of neighbouring pixel values. Second-ordered statistics are defined as the likelihood of observing a pair of grayscale (or intensity) values occurring at a random length with respect to the other. The length is randomly placed along with its orientation within the image. First and second ordered statistics are derived from each of the 9 abdominal ROIs.

A comprehensive set of first ordered statistical features commonly used in other studies was incorporated to depict the gross nature of the temperature distributions in the respective ROIs. The following was computed: mean, variance, skewness, and kurtosis (central moments), as well as the, minimum, maximum, median, mode, and the interquartile range (IQR).

Explored to a lesser extent was the use of more advanced second order statistical information that can be obtained from thermal images, using the grey level cooccurrence matrix (GLCM) [74], [95], [96], [97]. Derived for a grayscale image, this matrix computes how often a certain grey-level $i$ occurs horizontally, vertically, or diagonally with respect to the grey-level $j$. This represents the joint probability of two pixels having $i$-th and $j$-th intensity at a distance $d$, in varying distances and angles. This matrix provides information about the intensity distribution over the entire image, which allows the separation and classification of thermal images [97]. An example of how the GLCM was determined for the 4-by-4 image I is depicted in 
Figure 4-6. Here the image I has 4 grey-levels and a horizontal distance of 1 was considered.

\begin{tabular}{|c|c|c|c|c|c|c|c|}
\hline \multicolumn{4}{|c|}{ Image I } & \multicolumn{4}{|c|}{ GLCM } \\
\hline 0 & 0 & 1 & 1 & 2 & 2 & 2 & 0 \\
\hline 0 & 0 & 2 & 3 & 1 & 1 & 0 & 0 \\
\hline 2 & 3 & 3 & 1 & 0 & 0 & 0 & 2 \\
\hline 0 & 1 & 0 & 2 & 0 & 1 & 0 & 1 \\
\hline
\end{tabular}

Figure 4-6: An example of the GLCM created from the 4x4 image I with 4 grey-levels. The horizontal direction with distance 1 was used.

In this study the co-occurrence matrix was calculated for the 9 ROIs, neighbouring pixels $(\mathrm{d}=1)$ over different directions (horizontal, vertical, diagonal, and antidiagonal) and was averaged. The number of grey levels used to create the GLCM was 64. The same 9 first order and simple statistical features were computed for the GLCM images, providing second order statistical features.

For all first and second order statistical features extracted the absolute difference was computed for symmetric regions of the abdomen. The following comparisons were made: upper-to-lower (U-to-L), left-to-right (L-to-R), and the sum of difference for each quadrant to the whole abdomen (QTW). In this work, first order and second thermal symmetry analysis was performed for both the original and the enhanced thermal images. Enhanced images were as specified in section 4.3.2 Image Enhancement. Original thermal images used for the extraction first order thermal signatures were only rotated during pre-processing, thus differences were measured in degrees Celsius. Extraction of second order thermal signatures 
required normalization; consequently original thermal images were normalized. The figure below describes the process of thermal analysis performed on original and enhanced thermal images.

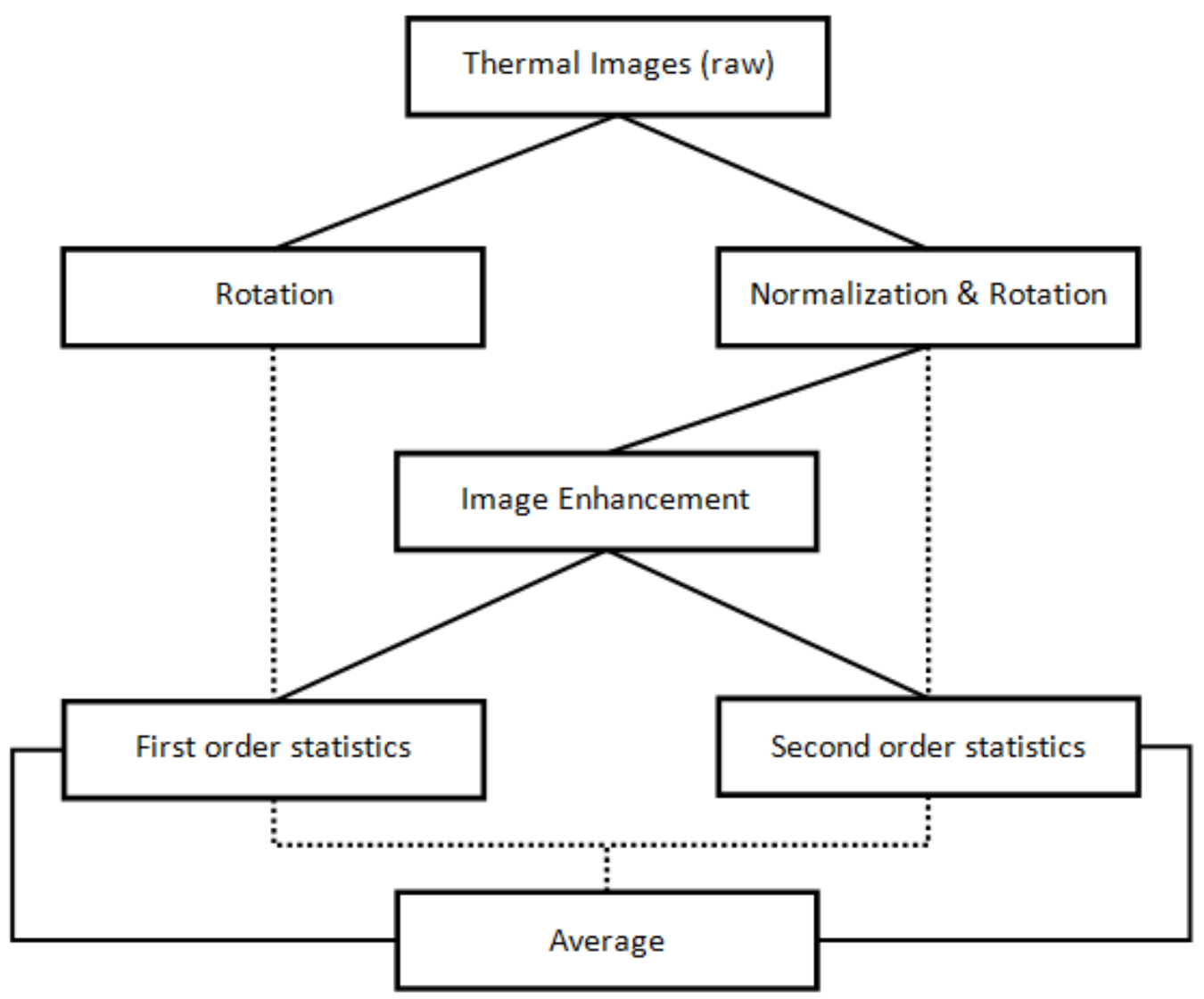

Figure 4-7: Flow diagram depicting the pre-processing, images enhancement, and analysis performed to create original and enhanced thermal images. After the pre-processing stage original images are indicated by dashed lines. First and second order thermal statistics were extracted from original and enhanced thermal images. Notice that original images were normalized when second order statistics were computed. The average of all U-to-L, L-to-R, and sum QTW differences of the statistical features extracted was then computed. 


\subsection{INTEGRATED SYSTEM}

Here, the objective was to integrate the various computerised techniques adopted into a full system. The GUI illustrated in Figure 4-8 was developed. To begin the user will load the data, by selecting the appropriate directory. Then, the list of images and the total number of images was displayed.

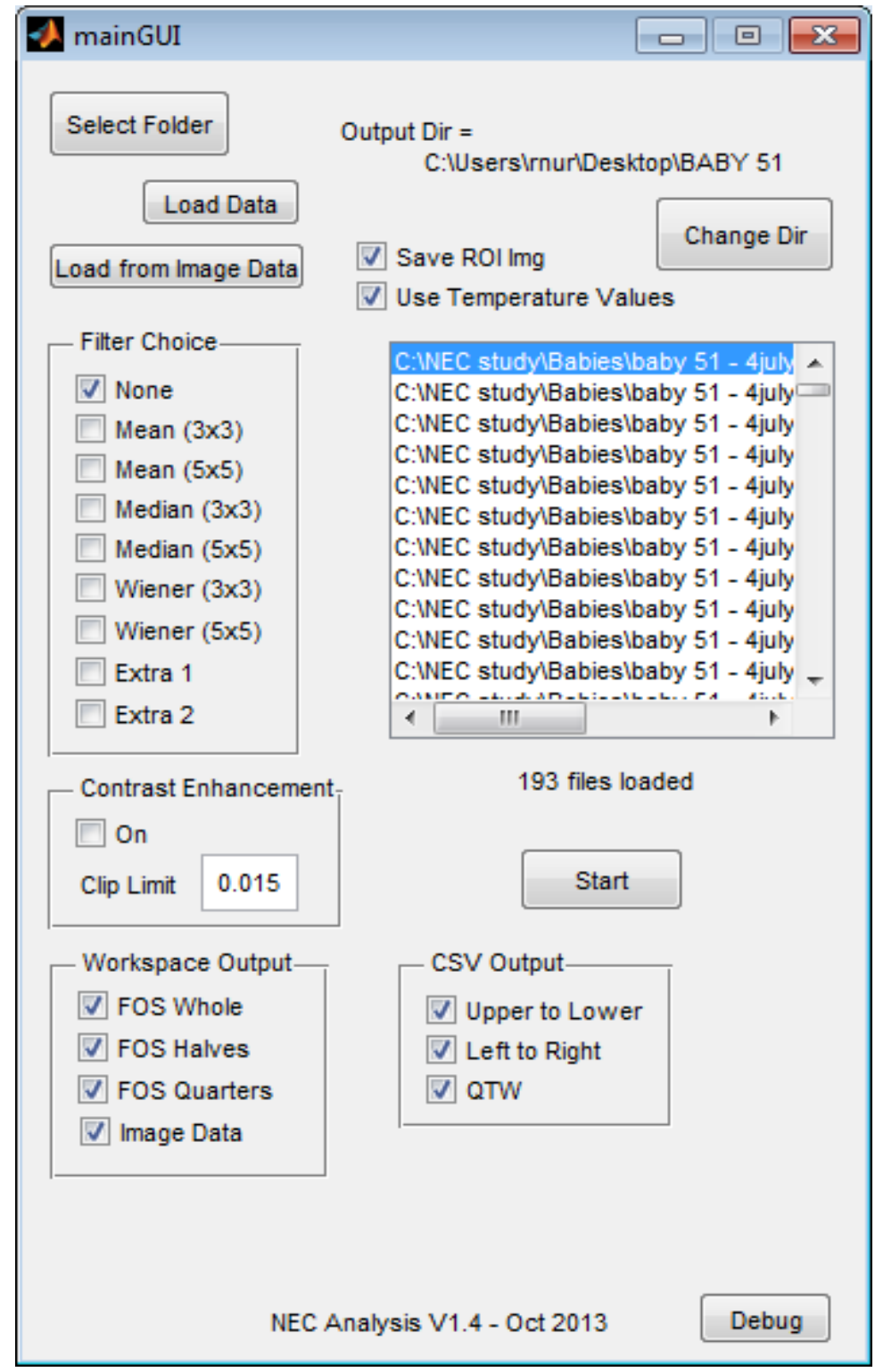

Figure 4-8: This GUI depicts the integrated system developed to perform computerized analysis of abdominal

infrared images. 
The user can then specify the desired image pre-processing and the enhancement parameters. This includes selecting one of the 6 filters available and determining the clip limit value to be used for contrast enhancement $\{0-1\}$. Once the output directory has been specified the start button can be pressed. The first image in the sequence appears as depicted in Figure 4-9, along with the prompt below in the MATLAB command window.
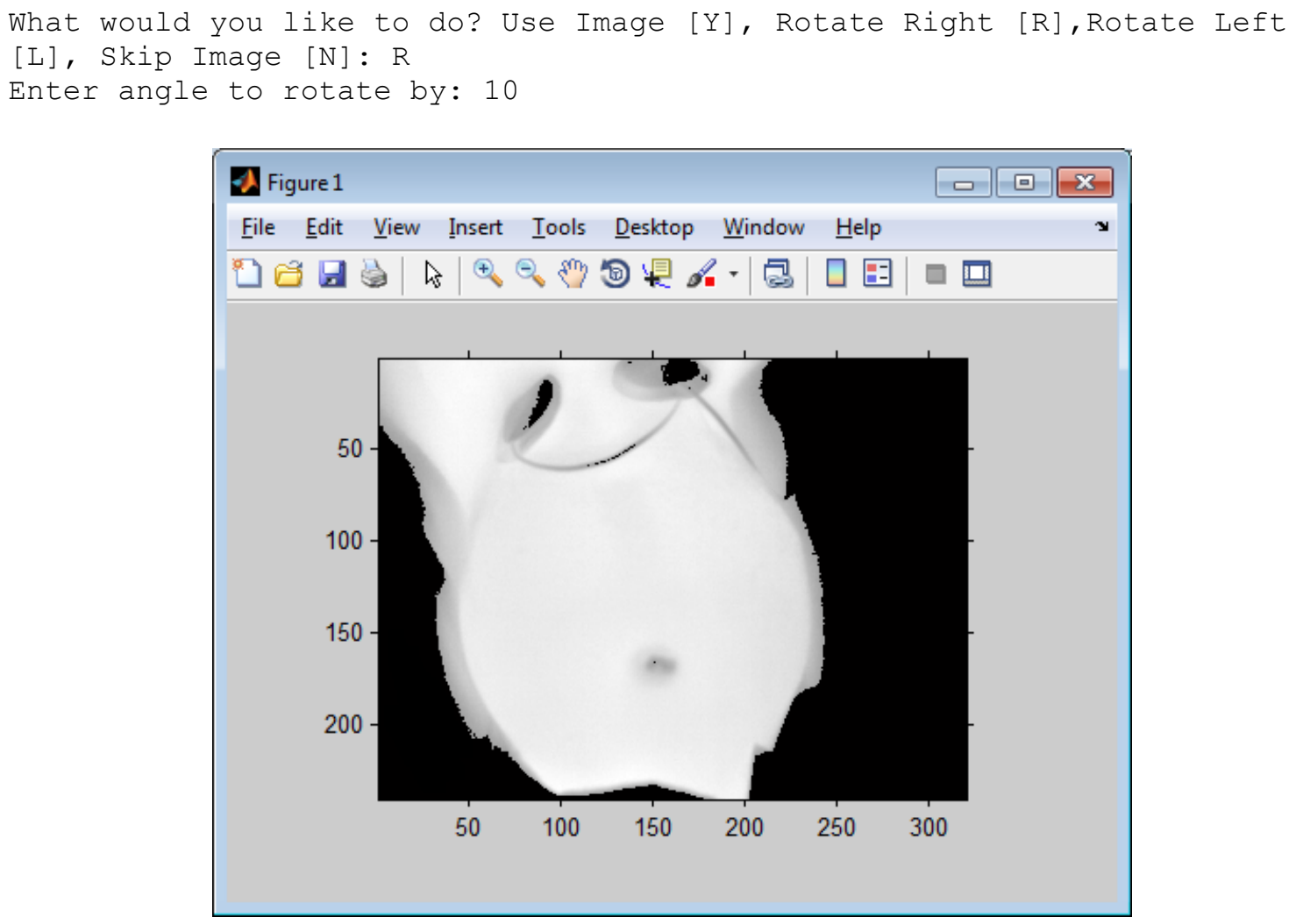

Figure 4-9: Initial thermal image before selection or processing, it was not normalized and no enhancement was performed. In this image it is evident that rotation to the right is required. The two cold spots on the top of the image are ECG electrodes.

The prompt provides the following options: image selection, rotation to the right, rotation to the left, or remove image from further analysis. For the image depicted in Figure 4-9 rotation to the right was selected, and another prompt appears to specify 
the number of degrees. The rotation of this image to the right 10 degrees corrects the orientation of the image, allowing for a more precise comparison of symmetric regions of skin temperatures (see Figure 4-10).

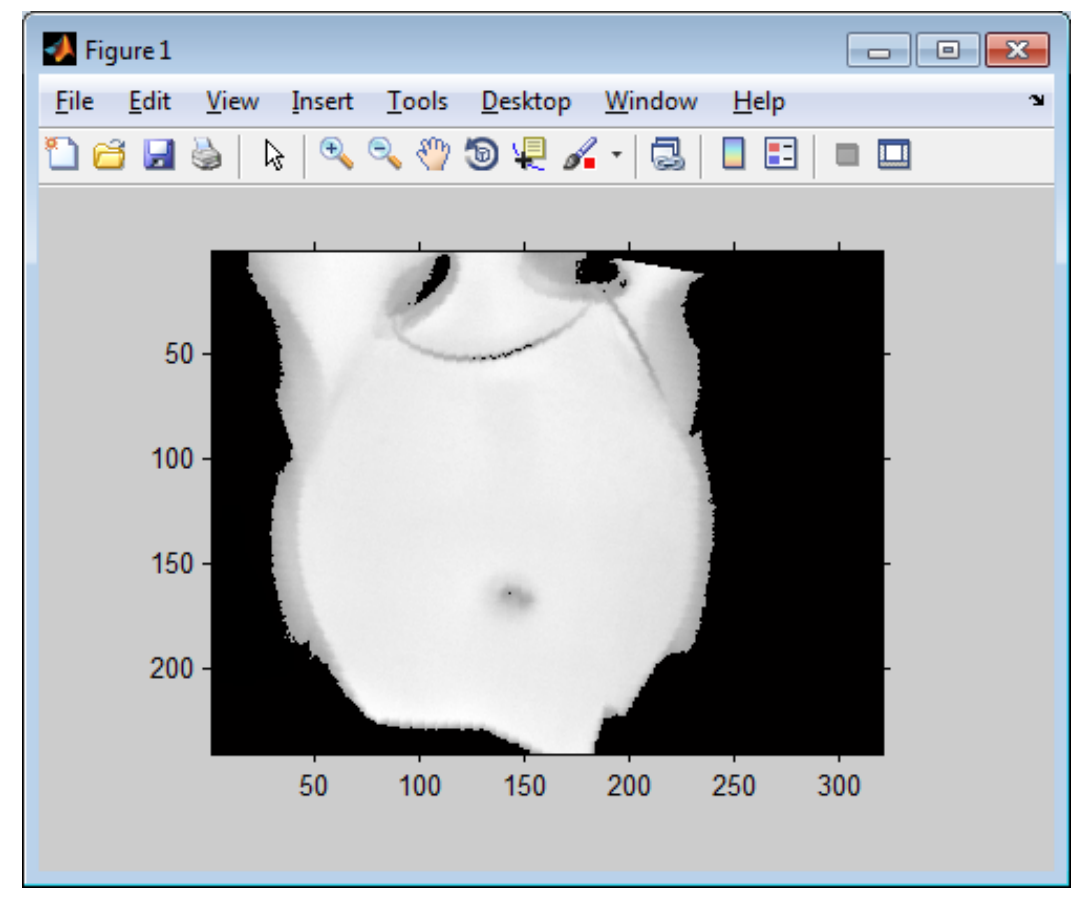

Figure 4-10: The original thermal image was rotated to the right by 10 degrees. The image now appears aligned.

Next, the user will perform manual ROI selection, as illustrated in Figure 4-5. The ROI does not need to be redrawn for each image since it remains intact; rather it is easily translated to adjust for motion. Once this process has been completed for all the images, thermal symmetry analysis begins. These results, along with all the data structures and images generated can be found in the output directory depicted in

Figure 4-11. Analysis can easily be repeated using different parameters by simply loading from image data and selecting the Image_Data file in the output directory. Image selection and ROI masks will be as defined previously. 


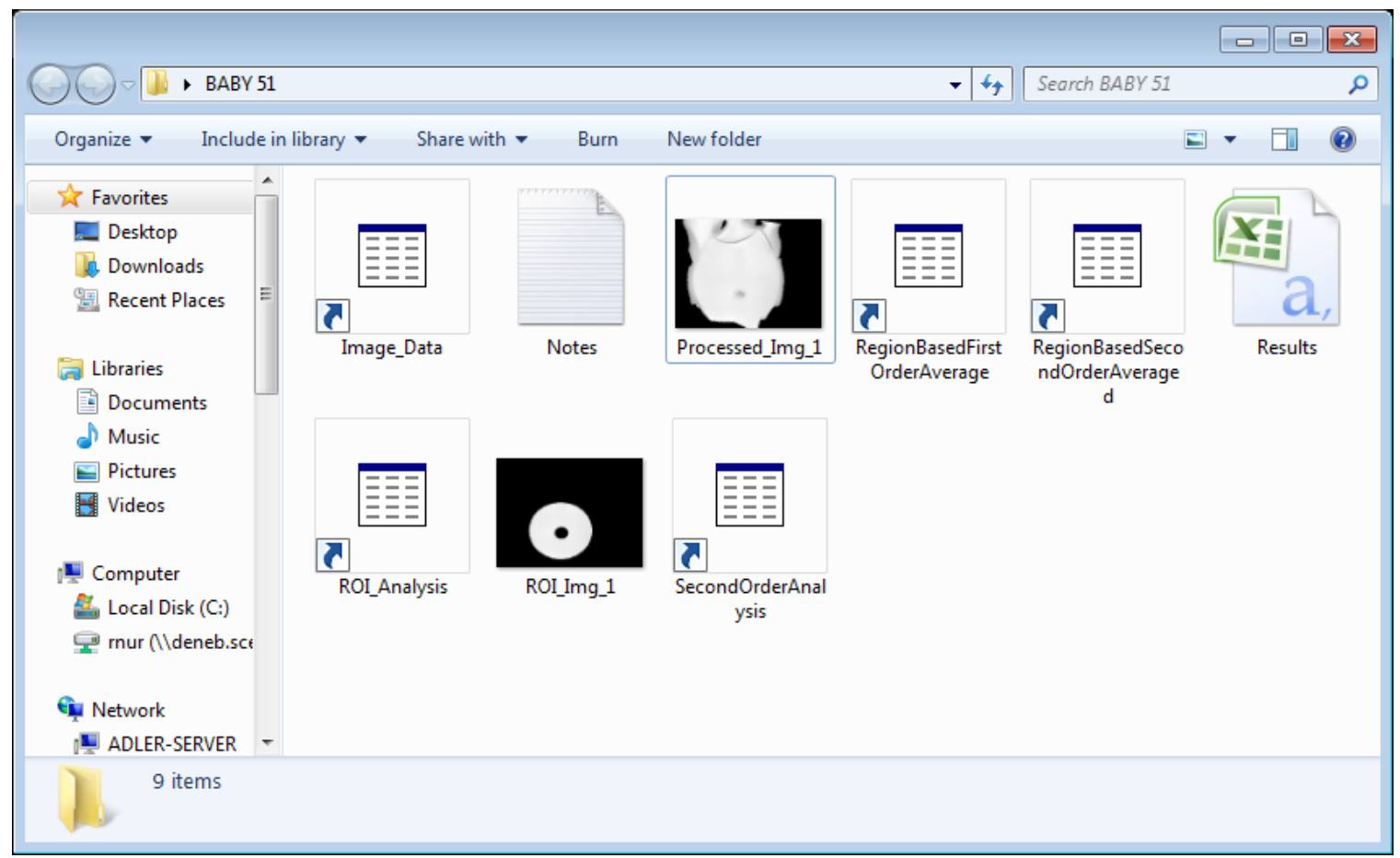

Figure 4-11: Example of the output directory when one image was used for thermal analysis. 


\section{Chapter 5: Results and Discussion}

The first part of Chapter 5 presents more details on the determination of the processing parameters selected for the image enhancement technique adopted in this thesis. The second part of this section discusses the results of statistical analysis performed, and then results of thermal symmetry testing are compared for the two groups. A final discussion will summarise the findings of this study.

\subsection{IMAGE ENHANCEMENT}

The aim of this section was to determine the parameters of the image enhancement techniques used in this work. The best filter was selected for noise reduction amongst those tested. Following, the appropriate clip-limit for CLAHE was determined, for both quantitative analysis and for the visual assessment of thermal images.

\subsubsection{NOISE REDUCTION}

To determine which noise reduction technique was the most suitable in this work, a simulation was conducted. The images were distorted with white Gaussian noise with a mean value of zero and variance of 0.001 ; as suggested in the literature [80], [98]. To assess the performance of an image restoration technique, a comparison between the original image $a[m, n]$ and the filtered image $\hat{a}[m, n]$ was performed. Comparisons were based on the mean square error (MSE) defined in equation 5-1. Here $M$ and $N$ represent the dimensions of the image, whereas $m$ and $n$ denote pixel coordinates.

$$
\left.E\{\widehat{a}, a\}=\frac{1}{M N} \sum_{m=0}^{M-1} \sum_{n=0}^{N-1}[\widehat{a}[m, n]-a[m, n]\}\right]^{2}
$$


Figure 5-1 depicts a comparison of the three noise reduction techniques. The selection criterion was based on the minimum MSE value of the filter that did not cause visible blurring. The performance of the mean filter was consistently the worst, achieving a minimum MSE value of 8.72 with a kernel size of $3 \times 3$. The best Wiener filter was a $(5 \times 5)$, which performed slightly better than the best median filter, also of size (5×5), with an MSE value of 5.22 in comparison to 5.66. All three filters produced visible blurring when a (7×7) kernel size was used. As a result, a Wiener $(5 \times 5)$ filter was chosen to perform noise reduction.

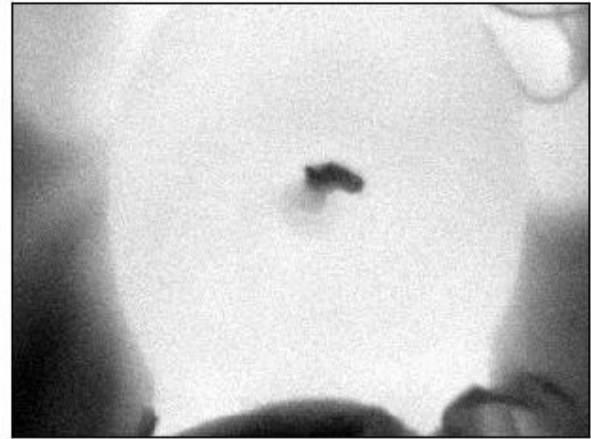

(a) Noisy Image, MSE $=29.83$

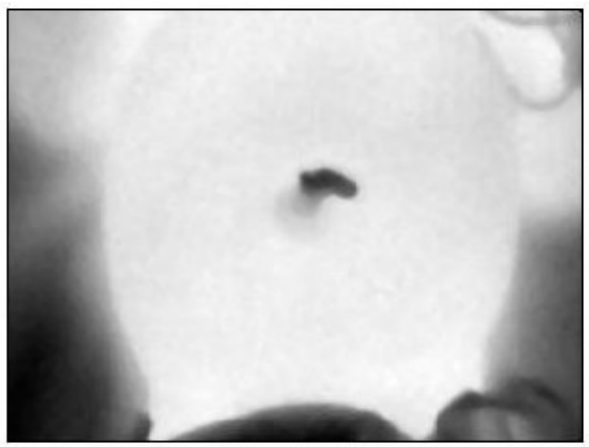

(c) Median Filter (5x5), MSE $=5.66$

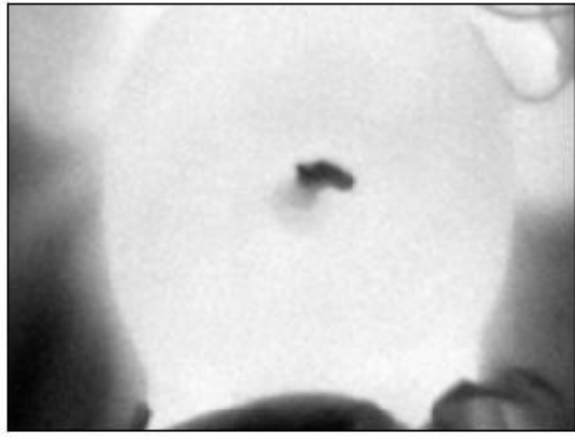

(b) Mean (3x3) Filter, MSE $=8.72$

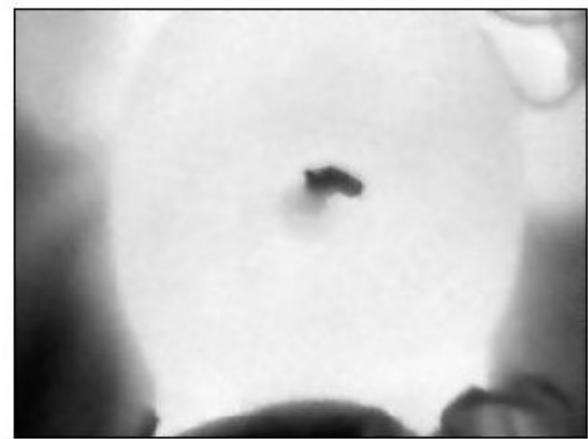

(d) Wiener Filter (5x5), MSE $=5.22$

Figure 5-1: Performance of mean, median, Wiener filter in removing white Gaussian Noise measured in MSE values. (a) An original thermal image that was normalized and with noise (b) - (d) Resulting images after noise reduction with the specified filters, only the best performing filter size of the three types tested is displayed. 


\subsubsection{CONTRAST ENHANCEMENT}

Similar to noise reduction, a simulation was also conducted to determine the appropriate clip-limit to be used to perform contrast enhancement, using CLAHE as performed in earlier work [93]. Figure 5-2 (a) depicts the outcome of Otsu's method of automatic segmentation based on grayscale intensities, following noise reduction using a Wiener filter $(5 \times 5)$. It is evident that this method was successful in removing the background while maintaining the body. Interestingly, the umbilical stump has also been segmented, and assumed to be of the same intensity as the background using this technique.

CLAHE was used to increase the contrast over the body region. For this reason contrasted enhanced images are compared to the segmented image depicted in Figure 5-2 (a), also using the MSE values to quantify how much they vary. In Figure 5-2 (b-f), it is evident that increasing the clip-limit results in an increase of the MSE value, as well as the local intensity variations within the area of interest. In this work clip-limit values of 0.005 and 0.01 were chosen as appropriate for performing asymmetry analysis [93]. This clip-limit increased the contrast to a level where the image remained accurate, and only subtle changes were noted. 


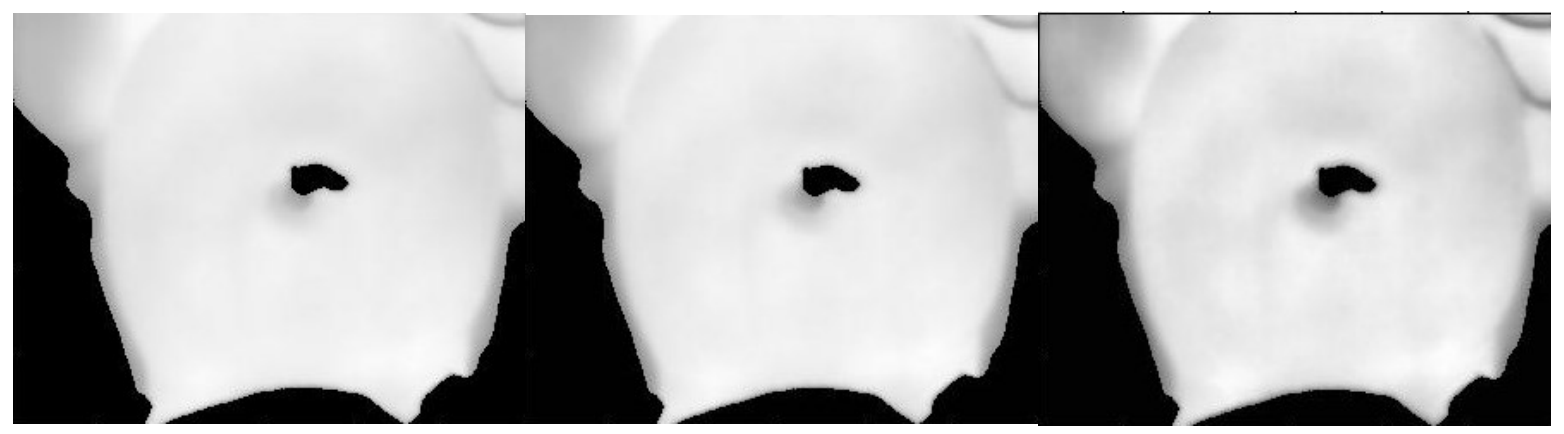

(a) Region of Interest Segmented using

(b) CLAHE (clip limit $=0.001$, Otsu's Algorithm MSE $=7.0886$

(c) CLAHE (clip limit $=0.005$ ), MSE $=54.3699$

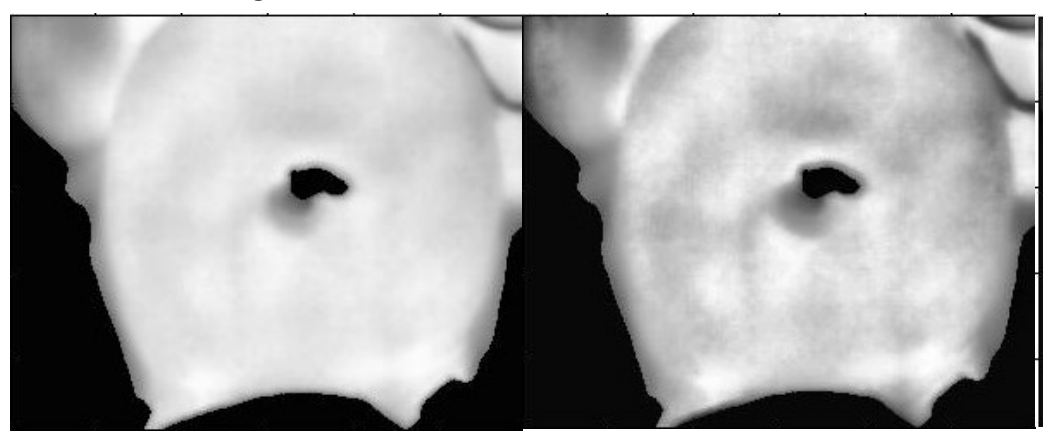

(d) CLAHE (clip limit $=0.01$ ) MSE $=99.9086$ MSE $=162.8720$ (e) CLAHE (clip limit $=0.03$ ),

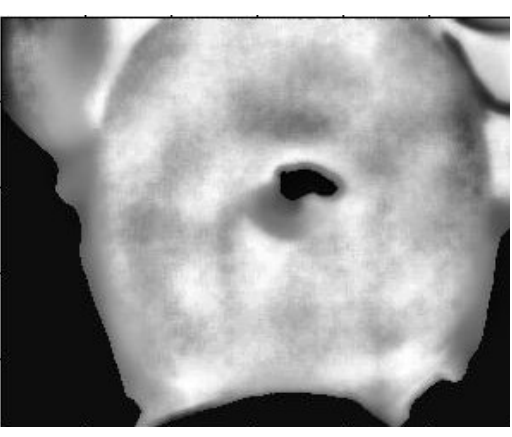

(f) CLAHE (clip limit $=0.05$ ), $\mathrm{MSE}=174.8905$

Figure 5-2: (a) Background removal using Otsu's Algorithm, performed on a normalized original thermal image (b) (f) CLAHE with varying clip limits, performed on (a). Subtle changes are noted in (c) and (d), whereas striking differences are noticed in (e) and (d). These images were captured from an infant with NEC.

In order to assess the influence of CLAHE on the visual perception of images, a cliplimit of 0.05 was selected. Figure 5-2 (f) and Figure 5-3(a) are enhanced images of infants with NEC, and Figure 5-3(b-c) are normal infants. In examining these images, a greater degree of thermal symmetry is noticed with the normal infants compared to the NEC infants. Figure 5-2 (f) depicts a thermal distribution that is warmer in the lower region of the abdomen, indicated by lighter pixel values (or higher intensity values), and cooler in the upper region indicated by a darker region (lower intensity values). Figure 5-3 (a) shows a temperature distribution that is also cooler in the upper region, but the warmest region is seen in the lower left quadrant. The abdominal thermal patterns in Figure 5-3 (b-c) appear to be symmetrically 
distributed upon visual examination of the left to right and the upper to lower regions.

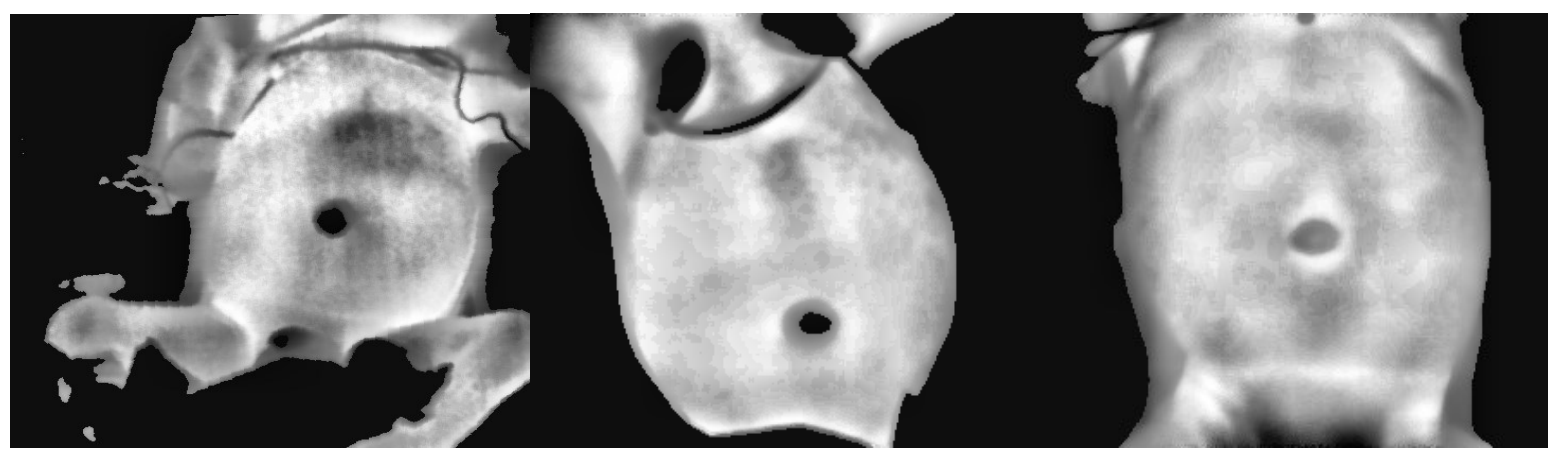

(a) NEC 2 CLAHE (clip limit $=0.05$ )

(b) Normal 1 CLAHE (clip limit $=0.05$ )

(c) Normal 2 CLAHE (clip limit $=0.05$ )

Figure 5-3: CLAHE (clip limit $=0.05$ ) performed on normalized original thermal images. (a) Images captured from an infant with NEC and (b-c) Normal infants

\subsection{DATA ANALYSIS}

The following section discusses the results of the thermal analysis of symmetry. This analysis was performed on the original thermal images and the enhanced grayscale images. Original images that were used to extract first order thermal signatures were not normalized; therefore thermal symmetry testing was measured in degrees Celsius. Whereas, the original images used to extract second order thermal signatures were normalized. Enhanced images were first smoothed with a Wiener (5x5) filter and then the contrast was enhanced using CLAHE with a clip limit of 0.005. Section 5.2.1 discusses the results of normality testing of the data sets. Section 5.2.2 reports on the results of statistical tests used to compare the distributions of values obtained from thermal symmetry testing for the groups (0Normal and 1-NEC). 


\subsubsection{LILLIEFORS TEST}

Prior to determining whether the two groups (0-Normal and 1-NEC) were statistically different, a Lilliefors test was applied to see if the datasets were of a normal distribution [99]. A logical value of $h=1$ was returned if the data was determined to not have a normal distribution at a significance level of $5 \%$, and $h=0$ when this assumption could not be rejected. The p-value represents the chance of error involved with the acceptance of the observation as truth; very large p-values indicate a high degree of uncertainty. The test produced varied results for the different features: the null hypothesis was both accepted and rejected. Results suggested a higher degree of uncertainty around the acceptance of the null hypothesis, indicated by high $\mathrm{p}$-values when $\mathrm{h}=0$. The rejection of the null hypothesis was done with more certainty, indicated by acceptable p-values (less than 0.05).

The results of this normality test computed for all 27 first order and simple statistical features for original and enhanced thermal images are shown in Table 5-1 and Table 5-2. In the cases were the h-value was 0 , indicating a normal distribution, the p-value ranged from 0.042 to 0.98 . High p-values cast doubt on the acceptance of the assumption made. When the h-value was 1 the p-values were lower than 0.043 , indicating a high degree of certainty.

Results of normality testing for the same 27 features extracted from the GLCMs (Grey-level co-occurrence matrix) computed for the original and enhanced thermal images are shown in Table 5-3 and Table 5-4. These tables show that the null hypothesis could not be rejected, p-values ranged from 0.055 to 0.92 . Again, high $\mathrm{p}$ - 
values indicate doubt with accepting the null hypothesis. When $h=1$ the $p$-values were less than 0.049 , indicating a high level of confidence with the acceptance of the assumption that the data did not have a normal distribution.

Table 5-1: Results of Lilliefors test of normality for all 27 first order and simple statistical features extracted from original thermal images.

\begin{tabular}{|l|l|l|l|l|}
\hline \multicolumn{5}{|c|}{ Lilliefors test } \\
\hline
\end{tabular}


Table 5-2: Results of Lilliefors test of normality computed for all 27 first order and simple statistical features extracted from enhanced thermal images.

\begin{tabular}{|l|l|l|l|l|}
\hline \multicolumn{5}{|c|}{ Lilliefors test } \\
\hline & \multicolumn{1}{|c|}{ NEC-value } & \multicolumn{2}{c|}{ h-value } & p-value \\
\hline Feature & \multicolumn{1}{|c|}{ h-value } & \multicolumn{1}{c|}{ Normal } \\
\hline U-to-L Mean & 0 & 0.059349 & 0 & 0.302656 \\
\hline L-to-R Mean & 0 & 0.104225 & 1 & 0.014779 \\
\hline QTW Mean & 1 & 0.014881 & 1 & 0.010543 \\
\hline U-to-L Median & 0 & 0.197709 & 0 & 0.640531 \\
\hline L-to-R Median & 0 & 0.121976 & 1 & 0.044695 \\
\hline QTW Median & 1 & 0.006326 & 1 & 0.037856 \\
\hline U-to-L Mode & 0 & 0.756489 & 0 & 0.161739 \\
\hline L-to-R Mode & 0 & 0.59579 & 0 & 0.053451 \\
\hline QTW Mode & 0 & 0.758274 & 0 & 0.375968 \\
\hline U-to-L Variance & 0 & 0.614952 & 0 & 0.172983 \\
\hline L-to-R Variance & 0 & 0.08643 & 1 & 0.012225 \\
\hline QTW Variance & 0 & 0.065614 & 0 & 0.092603 \\
\hline U-to-L Skewness & 0 & 0.597579 & 0 & 0.482437 \\
\hline L-to-R Skewness & 0 & 0.178017 & 0 & 0.277161 \\
\hline QTW Skewness & 0 & 0.239279 & 0 & 0.490822 \\
\hline U-to-L Kurtosis & 0 & 0.596499 & 0 & 0.483518 \\
\hline L-to-R Kurtosis & 0 & 0.179618 & 0 & 0.274595 \\
\hline QTW Kurtosis & 0 & 0.240732 & 0 & 0.490307 \\
\hline U-to-L IQR & 0 & 0.168468 & 0 & 0.090353 \\
\hline L-to-R IQR & 0 & 0.96029 & 0 & 0.135147 \\
\hline QTW IQR & 1 & 0.000833 & 1 & 0.016779 \\
\hline U-to-L Minimum & 1 & 0.01926 & 1 & 0.00195 \\
\hline L-to-R Minimum & 1 & 0.008885 & 1 & 0 \\
\hline QTW Minimum & 0 & 0.258353 & 0 & 0.640037 \\
\hline U-to-L Maximum & 0 & 0.42479 & 1 & 0.047891 \\
\hline L-to-R Maximum & 1 & 0.010131 & 1 & 0.001669 \\
\hline QTW Maximum & 1 & 0.003695 & 0 & 0.077613 \\
\hline & & & & \\
\hline
\end{tabular}


Table 5-3: Results of Lilliefors test of normality for all 27 first order and simple statistical features calculated from the GLCM of original thermal images.

\begin{tabular}{|c|c|c|c|c|}
\hline \multicolumn{5}{|c|}{ Lilliefors test } \\
\hline \multirow[b]{2}{*}{ Feature } & \multicolumn{2}{|c|}{ NEC } & \multicolumn{2}{|c|}{ Normal } \\
\hline & h-value & $\mathrm{p}$-value & h-value & $\mathrm{p}$-value \\
\hline U-to-L Mean & 0 & 0.180148 & 0 & 0.095489 \\
\hline L-to-R Mean & 0 & 0.925263 & 0 & 0.38893 \\
\hline QTW Mean & 0 & 0.590386 & 0 & 0.458934 \\
\hline U-to-L Median & 1 & 0 & 1 & 0 \\
\hline L-to-R Median & 1 & 0 & 1 & 0 \\
\hline QTW Median & 1 & 0 & 1 & 0 \\
\hline U-to-L Mode & 1 & 0 & 1 & 0 \\
\hline L-to-R Mode & 1 & 0 & 1 & 0 \\
\hline QTW Mode & 1 & 0 & 1 & 0 \\
\hline U-to-L Variance & 0 & 0.054289 & 1 & 0 \\
\hline L-to-R Variance & 0 & 0.108196 & 1 & 0 \\
\hline QTW Variance & 1 & 0.001502 & 1 & 0.00693 \\
\hline U-to-L Skewness & 0 & 0.268983 & 0 & 0.091383 \\
\hline L-to-R Skewness & 0 & 0.705908 & 1 & 0.002087 \\
\hline QTW Skewness & 0 & 0.612844 & 1 & 0.011107 \\
\hline U-to-L Kurtosis & 0 & 0.073069 & 1 & 0.003137 \\
\hline L-to-R Kurtosis & 0 & 0.206049 & 0 & 0.281253 \\
\hline QTW Kurtosis & 1 & 0.014979 & 1 & 0.041187 \\
\hline U-to-L IQR & 1 & 0 & 1 & 0 \\
\hline L-to-R IQR & 1 & 0 & 1 & 0 \\
\hline QTW IQR & 1 & 0 & 1 & 0 \\
\hline U-to-L Minimum & 1 & 0 & 1 & 0 \\
\hline L-to-R Minimum & 1 & 0 & 1 & 0 \\
\hline QTW Minimum & 1 & 0 & 1 & 0 \\
\hline U-to-L Maximum & 0 & 0.610423 & 1 & 0 \\
\hline L-to-R Maximum & 0 & 0.633779 & 0 & 0.185037 \\
\hline QTW Maximum & 0 & 0.271601 & 0 & 0.070541 \\
\hline
\end{tabular}


Table 5-4: Results of Lilliefors test of normality for all 27 first order and simple statistical features extracted from the GLCM of enhanced thermal images.

\begin{tabular}{|l|l|l|l|l|}
\hline \multicolumn{5}{|c|}{ Lilliefors test } \\
\hline \multicolumn{1}{|c|}{ Feature } & \multicolumn{1}{c|}{ p-value } & \multicolumn{2}{c|}{ h-value } & \multicolumn{1}{c|}{ p-value } \\
\hline & \multicolumn{1}{|c|}{ h-value } & \multicolumn{1}{c|}{ Normal } \\
\hline U-to-L Mean & 0 & 0.177725 & 0 & 0.096023 \\
\hline L-to-R Mean & 0 & 0.925946 & 0 & 0.388834 \\
\hline QTW Mean & 0 & 0.592275 & 0 & 0.459111 \\
\hline U-to-L Median & 1 & 0 & 1 & 0 \\
\hline L-to-R Median & 1 & 0 & 1 & 0 \\
\hline QTW Median & 1 & 0 & 1 & 0 \\
\hline U-to-L Mode & 1 & 0 & 1 & 0 \\
\hline L-to-R Mode & 1 & 0 & 1 & 0 \\
\hline QTW Mode & 1 & 0 & 1 & 0 \\
\hline U-To-L-Variance & 0 & 0.055072 & 1 & 0.000833 \\
\hline L-to-R Variance & 0 & 0.108891 & 1 & 0 \\
\hline QTW Variance & 1 & 0 & 1 & 0.004751 \\
\hline U-to-L Skewness & 0 & 0.269863 & 0 & 0.090909 \\
\hline L-to-R Skewness & 0 & 0.705293 & 1 & 0.002087 \\
\hline QTW Skewness & 0 & 0.61296 & 1 & 0.015159 \\
\hline U-to-L Kurtosis & 0 & 0.074105 & 1 & 0.003374 \\
\hline L-to-R Kurtosis & 1 & 0.208416 & 0 & 0.281653 \\
\hline QTW Kurtosis & 0 & 0.012721 & 1 & 0.041653 \\
\hline U-to-L IQR & 1 & 0 & 1 & 0 \\
\hline L-to-R IQR & 1 & 0 & 1 & 0 \\
\hline QTW IQR & 1 & 0 & 1 & 0 \\
\hline U-to-L Minimum & 0 & 0 & 1 & 0 \\
\hline L-to-R Minimum & 0 & 0 & 1 & 0 \\
\hline QTW Minimum & 0 & 0 & 1 & 0 \\
\hline U-to-L Maximum & 0.611415 & 1 & 0 \\
\hline L-to-R Maximum & 0.637242 & 0 & 0.18338 \\
\hline QTW Maximum & 0.273775 & 0 & 0.071309 \\
\hline
\end{tabular}

\subsubsection{TESTS OF STATISTICAL SIGNIFICANCE}

It was concluded that the data used in this study did not have a normal distribution;

for this reason, non-parametric hypothesis tests were used to determine if the two groups (0-Normal and 1-NEC) had statistically different results obtained from thermal analysis. The Wilcoxon Rank-Sum and Kruskal-Wallis tests were used. 
Given the NEC and the Normal data sets ( $\mathrm{x}$ and $\mathrm{y}$ ), the null hypothesis tested was that the independent samples were of an identical continuous distribution with equivalent medians [100], [101], [102]. A significance level of 0.05 is used in this study.

The results of these tests for all 27 first order and simple statistical features calculated from original thermal images are shown in Table 5-5. The null hypothesis was rejected for 9 of the 27 features, indicating that these features of the two groups (0-Normal and 1-NEC) were determined not to be from an identical continuous distribution, with equal medians. These features were the: U-to-L Means, QTW Means, U-to-L Medians, QTW Medians, U-to-L Modes, QTW Mode, U-to-L Variance, QTW Variance, and QTW IQR. Box plots illustrated in Figure 5-4 to Figure 5-12 compare the distributions of the 9 features for the two groups; a red star denotes an outlier value. Across all features, the spread in the distribution of the NEC data in comparison to the normal was larger.

The QTW Variance, U-to-L Medians, and U-to-L Means had the lowest p-values: $0.00135,0.00298$, and 0.00347 respectfully. The remaining values ranged from 0.00541 to 0.0381 . Note that only comparisons of the U-to-L difference of temperatures and sum of QTW differences of temperature were deemed to have differing medians and continuous distributions for the two groups (0-Normal and 1NEC). With 4 out the 9 features being U-to-L features and 5 were sum of QTW differences. 
Table 5-5: The Wilcoxon Rank-Sum and Kruskal-Wallis tests performed for all 27 first order and simple statistical featured using original thermal images. The rank is based on the ascending order of $p$-values, when $h=1$.

\begin{tabular}{|c|c|c|c|c|}
\hline \multirow[b]{2}{*}{ Feature } & \multicolumn{2}{|c|}{ Wilcoxon Rank-Sum } & \multirow{2}{*}{$\begin{array}{l}\text { Kruskal-Wallis Test } \\
\text { p-value }\end{array}$} & \multirow[t]{2}{*}{ Rank } \\
\hline & $\mathrm{p}$-value & h-value & & \\
\hline U-to-L Mean & 0.003742 & 1 & 0.003470017 & 3 \\
\hline L-to-R Mean & 0.868951 & 0 & 0.850436268 & \\
\hline QTW Mean & 0.028377 & 1 & 0.026718823 & 8 \\
\hline U-to-L Median & 0.003216 & 1 & 0.002979467 & 2 \\
\hline L-to-R Median & 0.906186 & 0 & 0.887537084 & \\
\hline QTW Median & 0.015194 & 1 & 0.014233988 & 6 \\
\hline U-to-L Mode & 0.019624 & 1 & 0.018422125 & 7 \\
\hline L-to-R Mode & 0.75929 & 0 & 0.741412683 & \\
\hline QTW Mode & 0.005821 & 1 & 0.00541439 & 4 \\
\hline U-to-L Variance & 0.040305 & 1 & 0.038062607 & 9 \\
\hline L-to-R Variance & 0.868951 & 0 & 0.850436268 & \\
\hline QTW Variance & 0.001463 & 1 & 0.001348043 & 1 \\
\hline U-to-L Skewness & 0.194851 & 0 & 0.186857503 & \\
\hline L-to-R Skewness & 0.654273 & 0 & 0.637351888 & \\
\hline QTW Skewness & 0.494268 & 0 & 0.479500122 & \\
\hline U-to-L Kurtosis & 0.194851 & 0 & 0.186857503 & \\
\hline L-to-R Kurtosis & 0.654273 & 0 & 0.637351888 & \\
\hline QTW Kurtosis & 0.494268 & 0 & 0.479500122 & \\
\hline U-to-L IQR & 0.094232 & 0 & 0.089686022 & \\
\hline L-to-R IQR & 0.409395 & 0 & 0.396143909 & \\
\hline QTW IQR & 0.010195 & 1 & 0.009521891 & 5 \\
\hline U-to-L Minimum & 0.494268 & 0 & 0.479500122 & \\
\hline L-to-R Minimum & 0.75929 & 0 & 0.741412683 & \\
\hline QTW Minimum & 0.409395 & 0 & 0.396143909 & \\
\hline U-to-L Maximum & 0.383155 & 0 & 0.370429791 & \\
\hline L-to-R Maximum & 1 & 0 & 1 & \\
\hline QTW Maximum & 0.211584 & 0 & 0.203091788 & \\
\hline
\end{tabular}




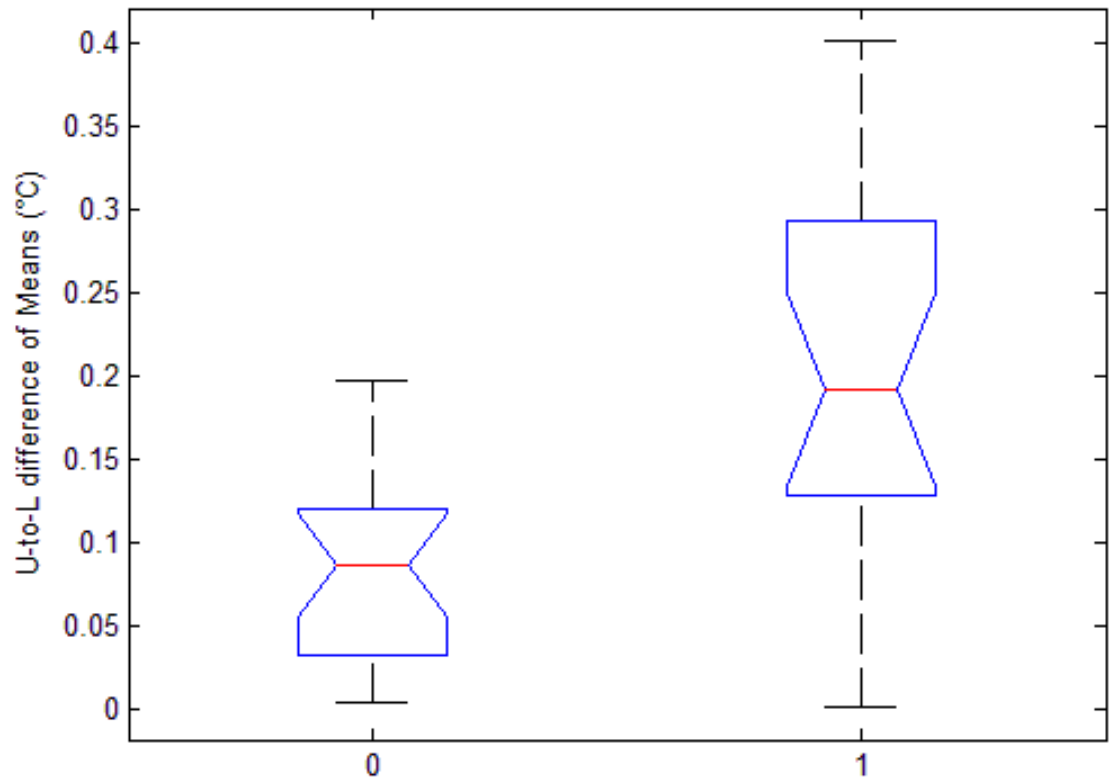

Figure 5-4: Box plot for U-to-L difference of means $\left({ }^{\circ} \mathrm{C}\right)(0$-Normal, 1-NEC) from original thermal images.

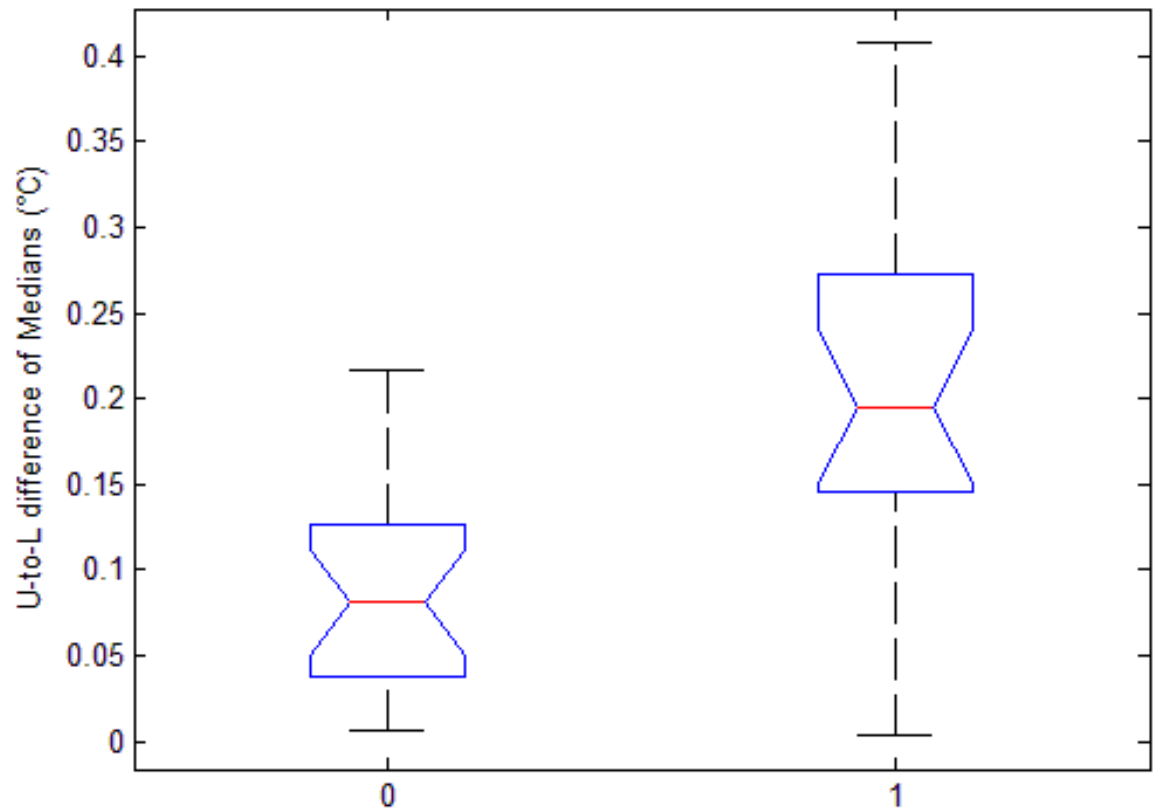

Figure 5-5: Box plot for U-to-L difference of medians $\left({ }^{\circ} \mathrm{C}\right)(0$-Normal, 1-NEC) from original thermal images. 


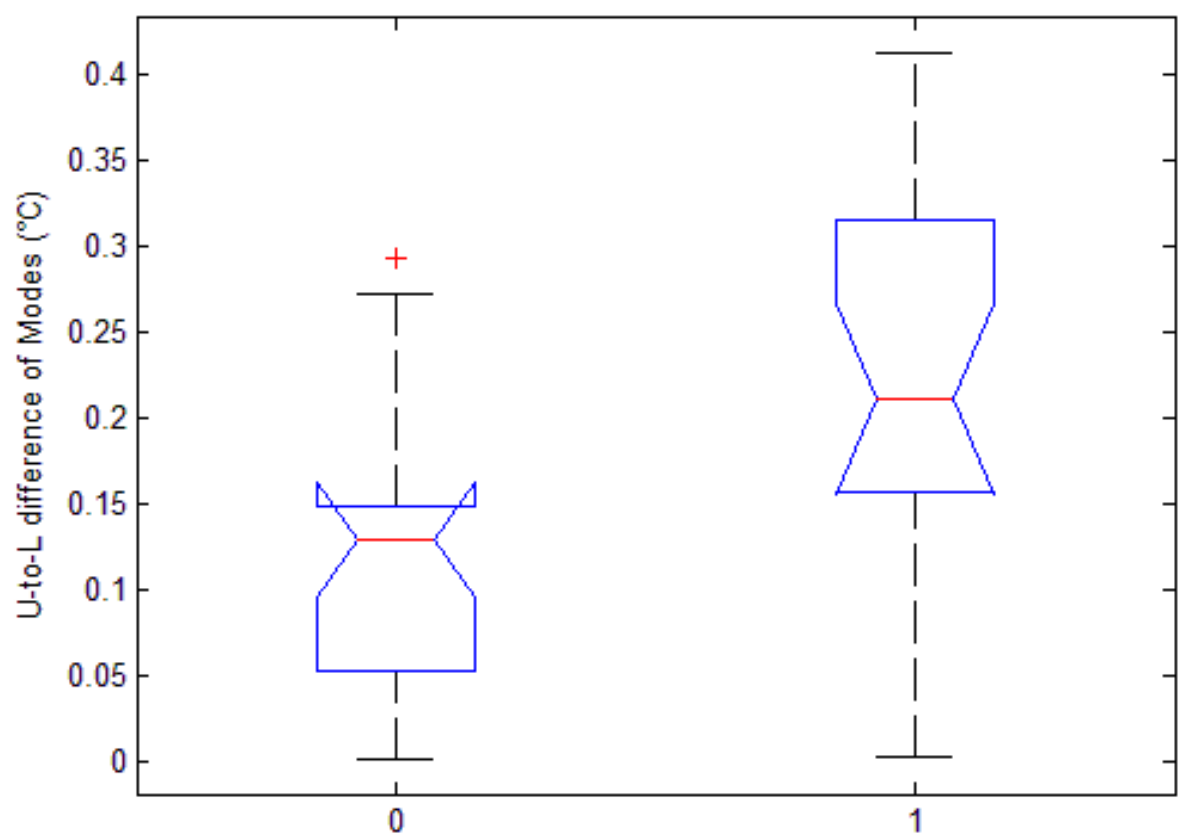

Figure 5-6: Box plot for U-to-L difference of Modes $\left({ }^{\circ} \mathrm{C}\right)(0$-Normal, 1-NEC) from original thermal images.

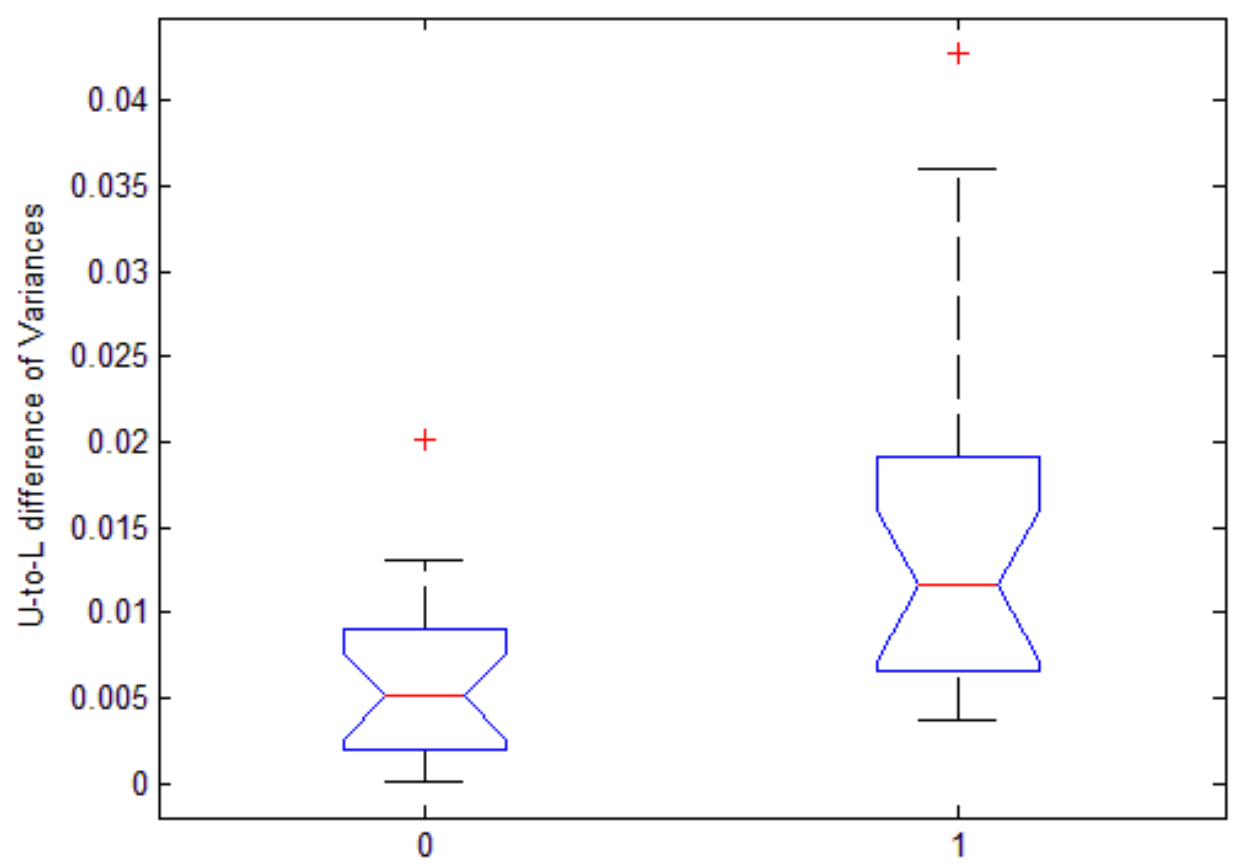

Figure 5-7: Box plot for U-to-L difference of variances $\left({ }^{\circ} \mathrm{C}\right)(0$-Normal, 1-NEC) from original thermal images. 


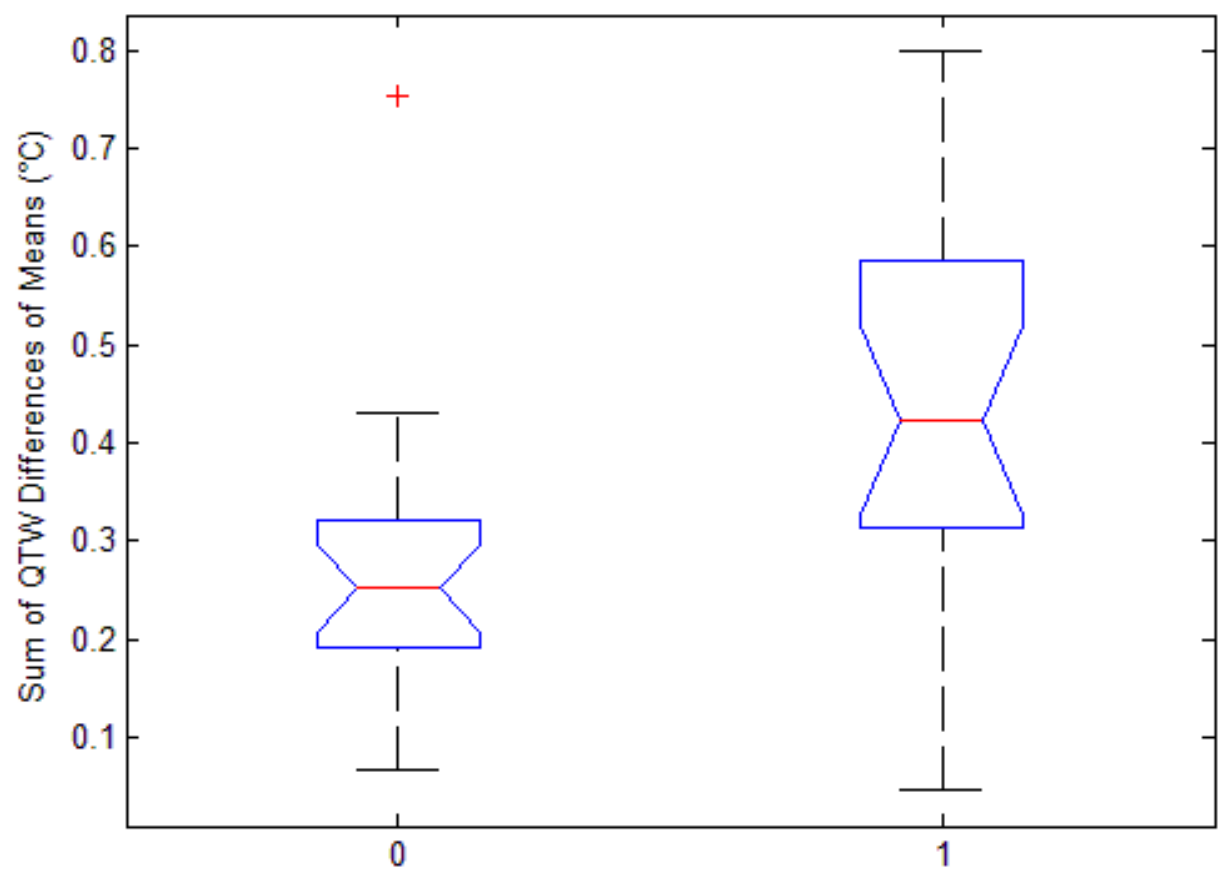

Figure 5-8: Box plot of the sum of QTW difference of means $\left({ }^{\circ} \mathrm{C}\right)(0$-Normal, 1-NEC) from original thermal images.

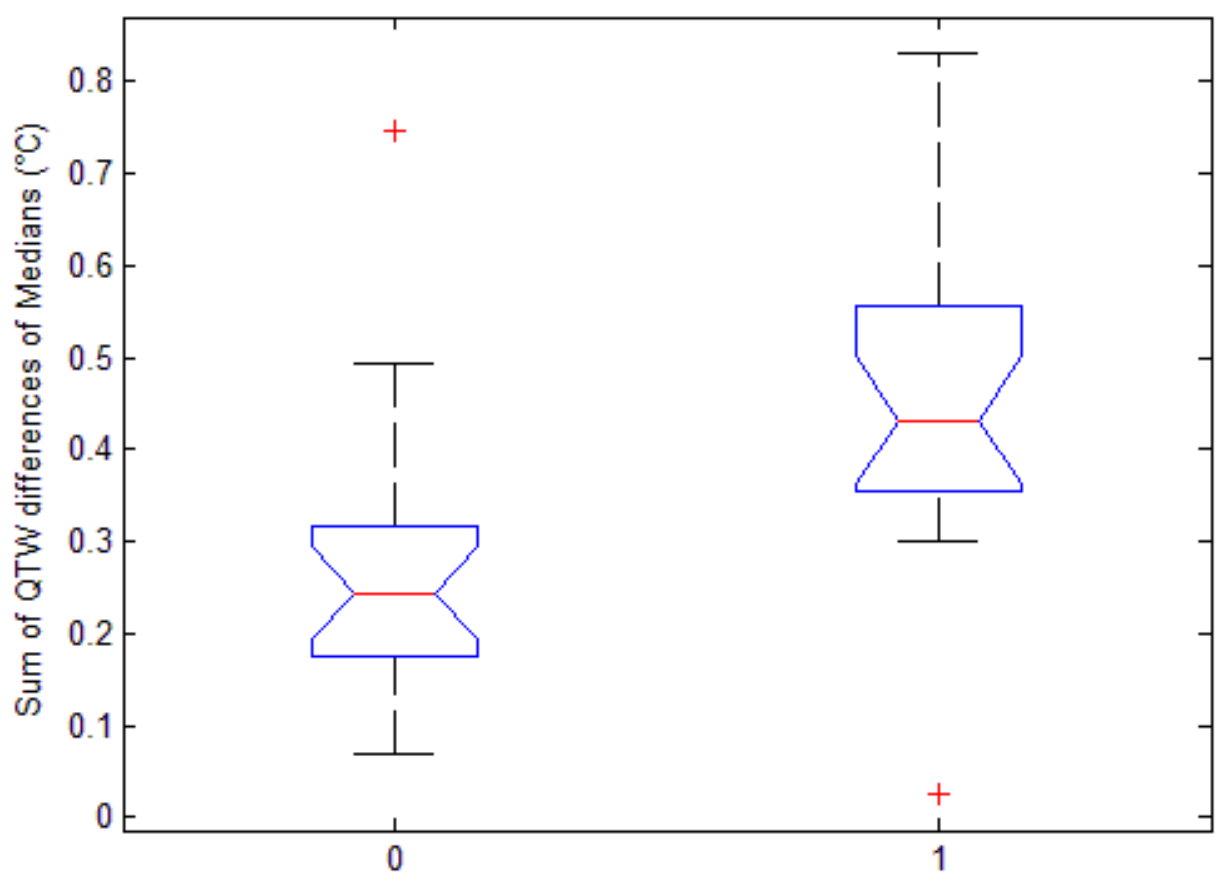

Figure 5-9: Box plot of the sum of QTW difference of medians $\left({ }^{\circ} \mathrm{C}\right)(0$-Normal, 1-NEC) from original thermal images. 


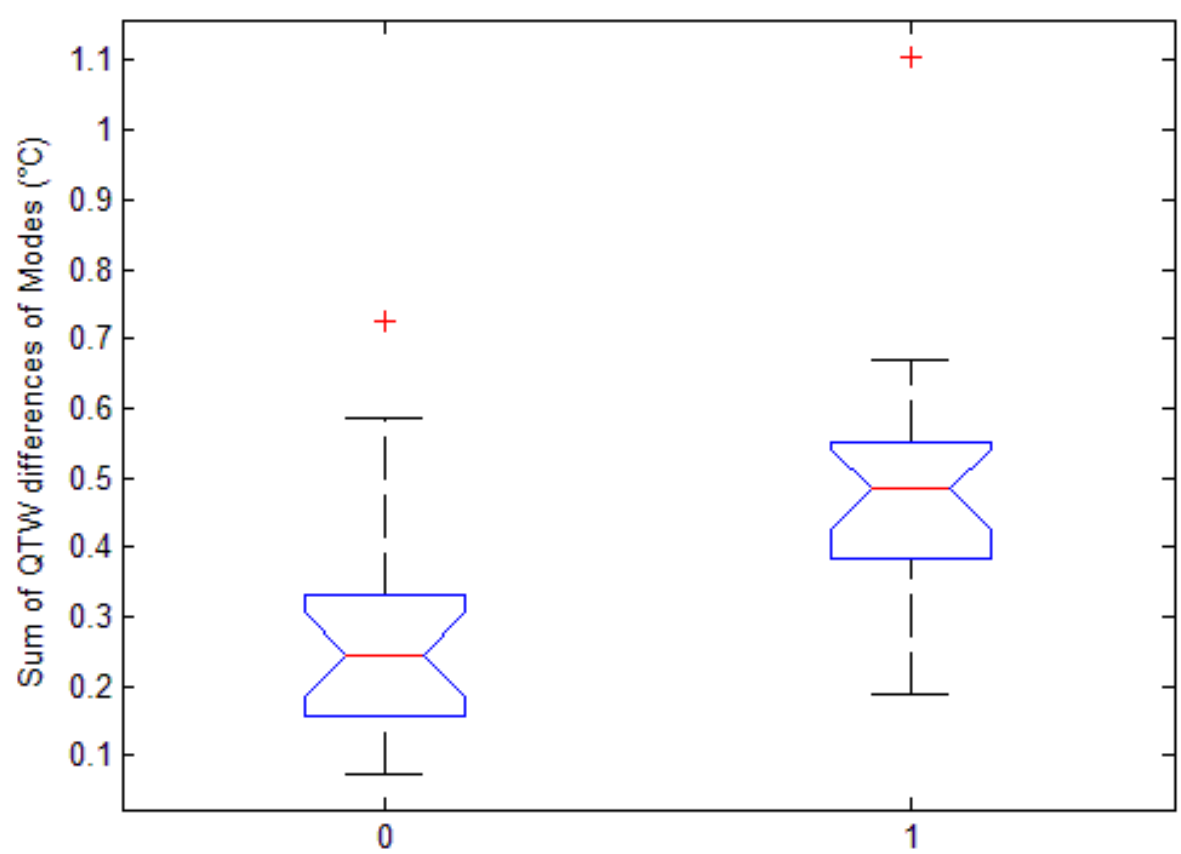

Figure 5-10: Box plot of the sum of QTW difference of modes $\left({ }^{\circ} \mathrm{C}\right)(0$-Normal, 1-NEC) from original thermal images.

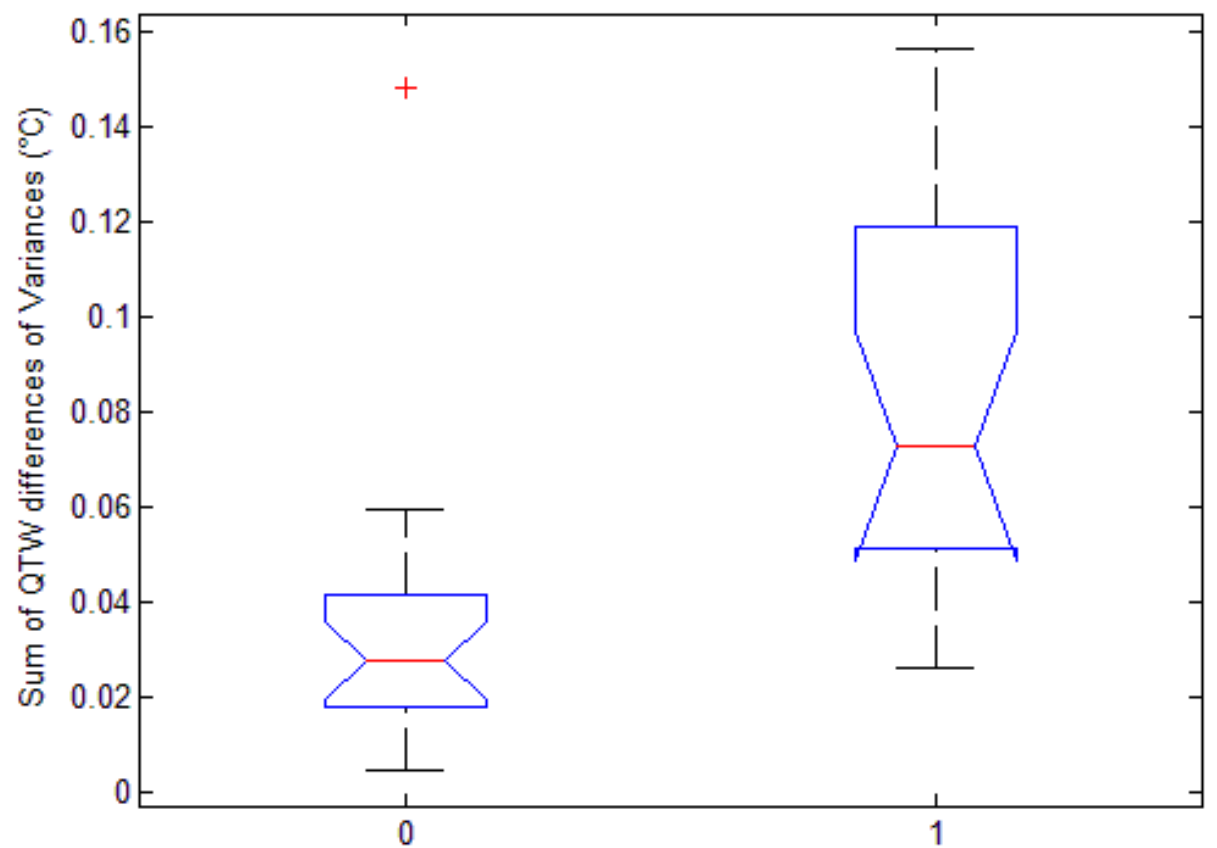

Figure 5-11: Box plot of the sum of QTW difference of variances $\left({ }^{\circ} \mathrm{C}\right)(0$-Normal, 1-NEC) from original thermal images. 


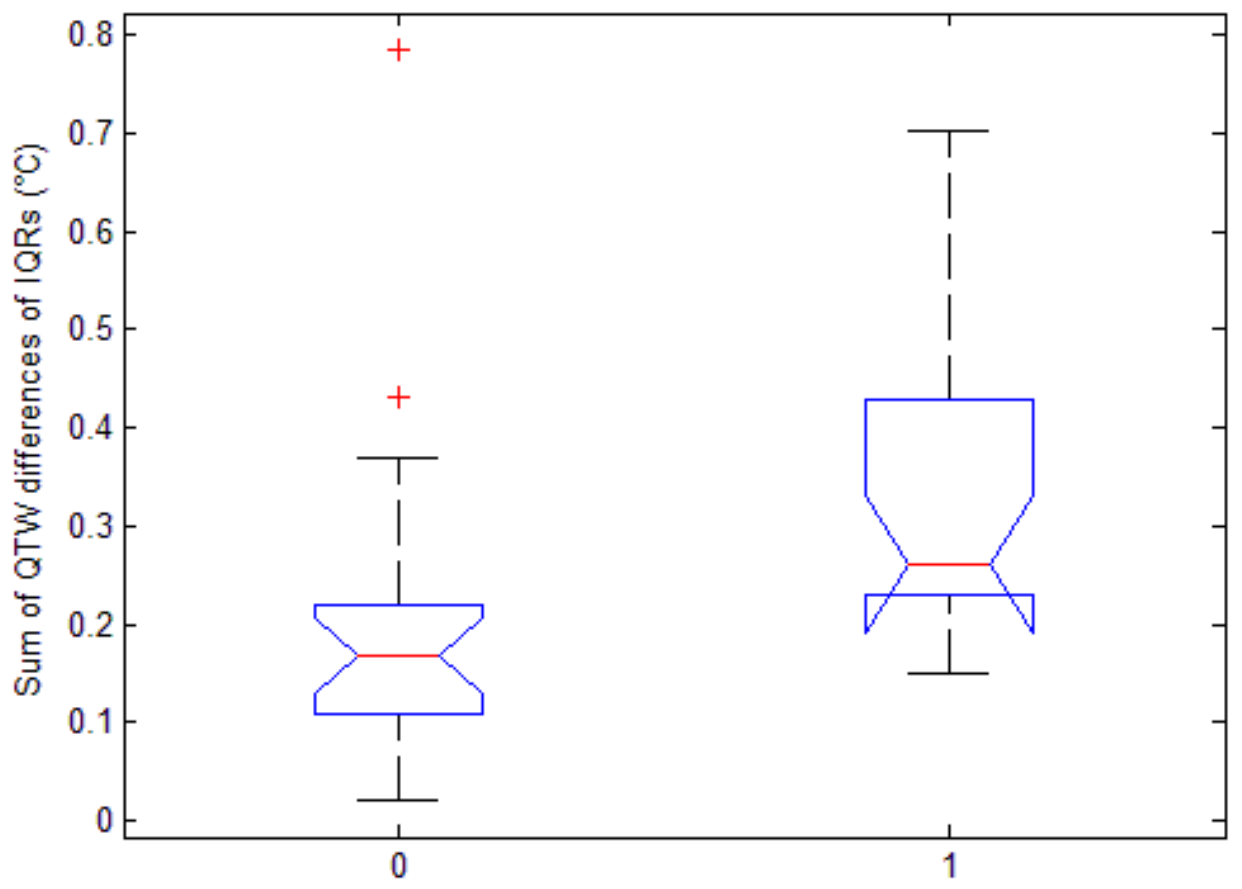

Figure 5-12: Box plot of the sum of QTW difference of IQRs $\left({ }^{\circ} \mathrm{C}\right)(0$-Normal, 1-NEC) from original thermal images.

The results of the Wilcoxon Rank-Sum and Kruskal-Wallis tests performed for all 27 first-order and simple statistical features for the enhanced thermal images, is shown in Table 5-6. The null hypothesis was not accepted for 12 of the 27 features tested. These features were: U-to-L Mean, QTW Mean, U-to-L Median, QTW Median, U-to-L Mode, QTW Mode, U-to-L Variance, QTW Variance, U-to-L Skewness, U-to-L Kurtosis, U-to-L IQR, and QTW IQR. Box plots of these 12 features for the two groups are illustrated from Figure 5-13 to Figure 5-24. Across all features, the distribution of the NEC data in comparison to the normal had a higher spread of values.

The QTW Variance, QTW Means, QTW Medians, U-to-L Variance, U-to-L IQR, QTW IQR had the lowest p-values, of: $0.000817,0.00135,0.00255,0.00298,0.00347$, and 
0.00347. The remaining 6 features had p-values that ranged from 0.0109 to 0.0427 .

Again, only comparisons of the U-to-L differences and sum of QTW differences were deemed to have differing medians and continuous distributions for the two groups (0-Normal and 1-NEC). Of the 12 features, 7 were U-to-L differences and 5 were sum of QTW differences.

Table 5-6: The Wilcoxon Rank-Sum and Kruskal-Wallis tests performed for all 27 first order and simple statistical featured using enhanced thermal images. The rank is based on the ascending order of p-values, when $h=1$.

\begin{tabular}{|l|l|l|l|l|}
\hline & \multicolumn{2}{|l|}{ Wilcoxon Rank-Sum } & Kruskal-Wallis Test & Rank \\
\hline Feature & p-value & h-value & p-value & \\
\hline U-to-L Mean & 0.011669 & 1 & 0.010909498 & 7 \\
\hline L-to-R Mean & 0.333854 & 0 & 0.322198806 & \\
\hline QTW Mean & 0.001463 & 1 & 0.001348043 & 2 \\
\hline U-to-L Median & 0.045127 & 1 & 0.042658379 & 12 \\
\hline L-to-R Median & 0.211584 & 0 & 0.203091788 & \\
\hline QTW Median & 0.002759 & 1 & 0.002552991 & 3 \\
\hline U-to-L Mode & 0.031962 & 1 & 0.030123644 & 8 \\
\hline L-to-R Mode & 0.436677 & 0 & 0.422907242 & \\
\hline QTW Mode & 0.031962 & 1 & 0.030123644 & 9 \\
\hline U-to-L Variance & 0.003216 & 1 & 0.002979467 & 4 \\
\hline L-to-R Variance & 0.050426 & 0 & 0.04771488 & \\
\hline QTW Variance & 0.000889 & 1 & 0.000816994 & 1 \\
\hline U-to-L Skewness & 0.031962 & 1 & 0.030123644 & 10 \\
\hline L-to-R Skewness & 0.524518 & 0 & 0.509275437 & \\
\hline QTW Skewness & 0.164334 & 0 & 0.157299207 & \\
\hline U-to-L Kurtosis & 0.031962 & 1 & 0.030123644 & 11 \\
\hline L-to-R Kurtosis & 0.524518 & 0 & 0.509275437 & \\
\hline QTW Kurtosis & 0.164334 & 0 & 0.157299207 & \\
\hline U-to-L IQR & 0.003742 & 1 & 0.003470017 & 5 \\
\hline L-to-R IQR & 0.050426 & 0 & 0.04771488 & \\
\hline QTW IQR & 0.003742 & 1 & 0.003470017 & 6 \\
\hline U-to-L Minimum & 0.383155 & 0 & 0.370429791 & \\
\hline L-to-R Minimum & 0.114289 & 0 & 0.108984256 & \\
\hline QTW Minimum & 0.211584 & 0 & 0.203091788 & \\
\hline U-to-L Maximum & 0.094232 & 0 & 0.089686022 & \\
\hline L-to-R Maximum & 0.868951 & 0 & 0.850436268 & \\
\hline QTW Maximum & 0.114289 & 0 & 0.108984256 & \\
\hline
\end{tabular}




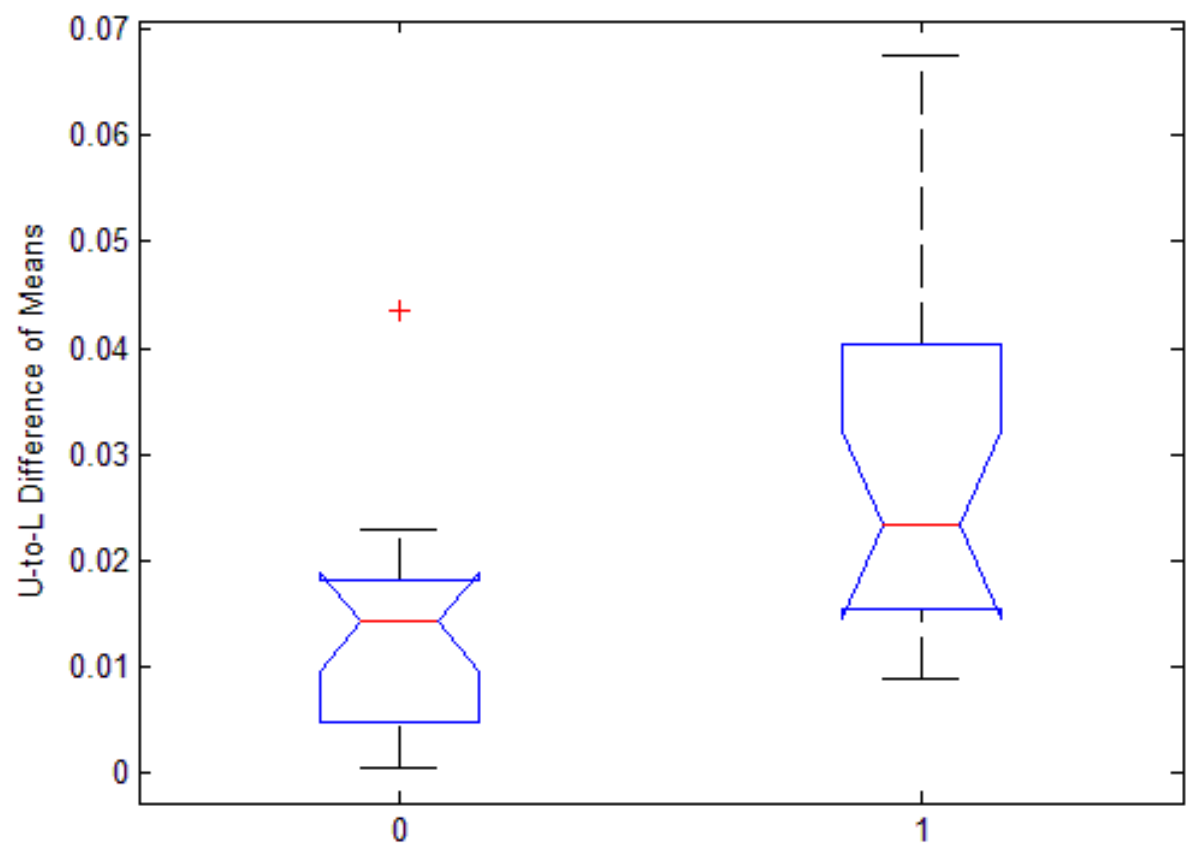

Figure 5-13: Box plot of U-to-L difference of Means (0-Normal, 1-NEC) from enhanced thermal images.

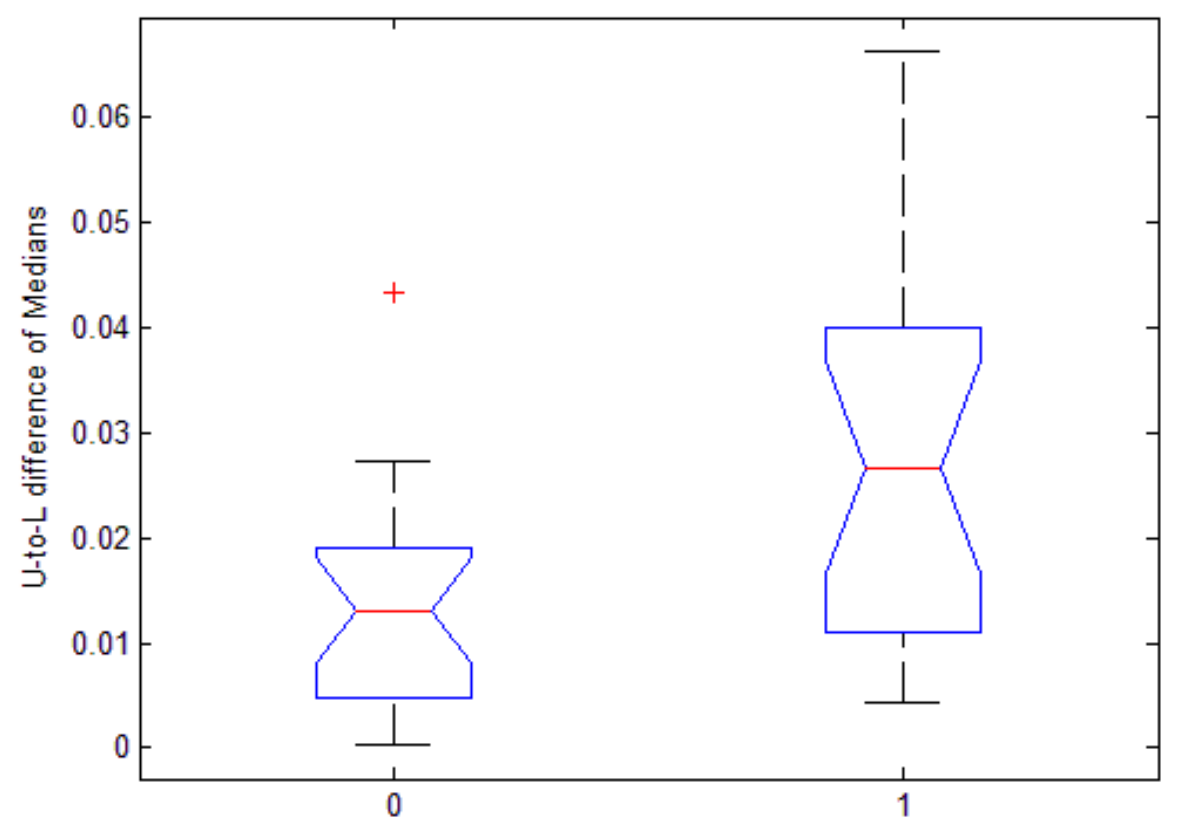

Figure 5-14: Box plot of U-to-L difference of Medians (0-Normal, 1-NEC) from enhanced thermal images. 


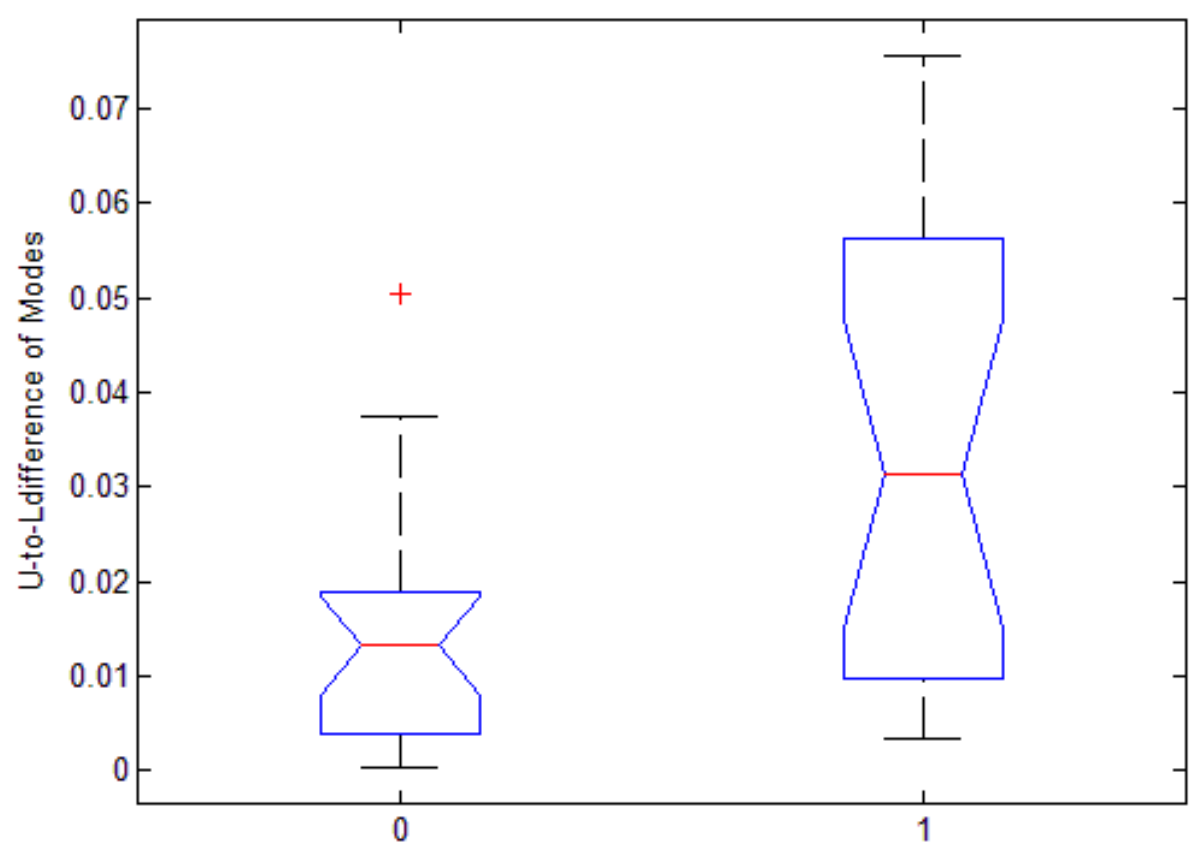

Figure 5-15: Box plot of U-to-L difference of Modes (0-Normal, 1-NEC) from enhanced thermal images.

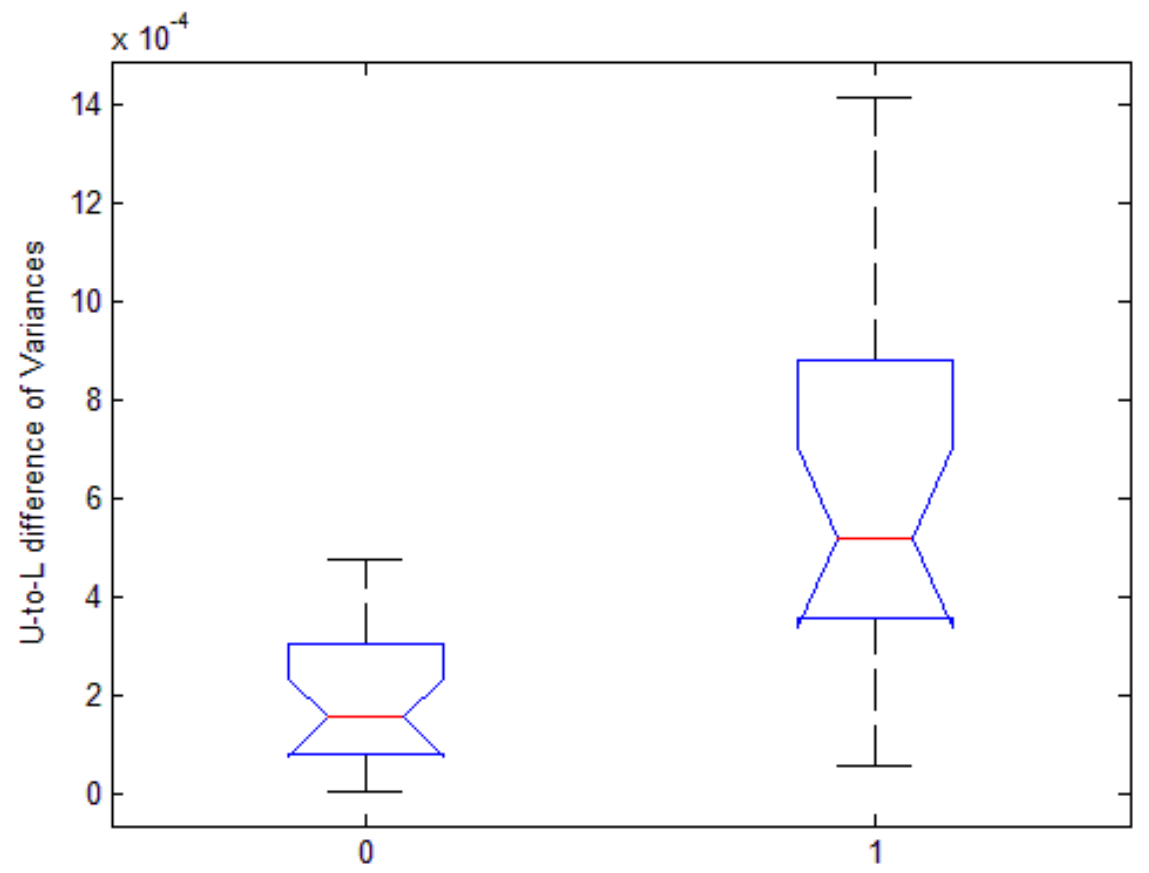

Figure 5-16: Box plot of U-to-L difference of Variances (0-Normal, 1-NEC) from enhanced thermal images. 


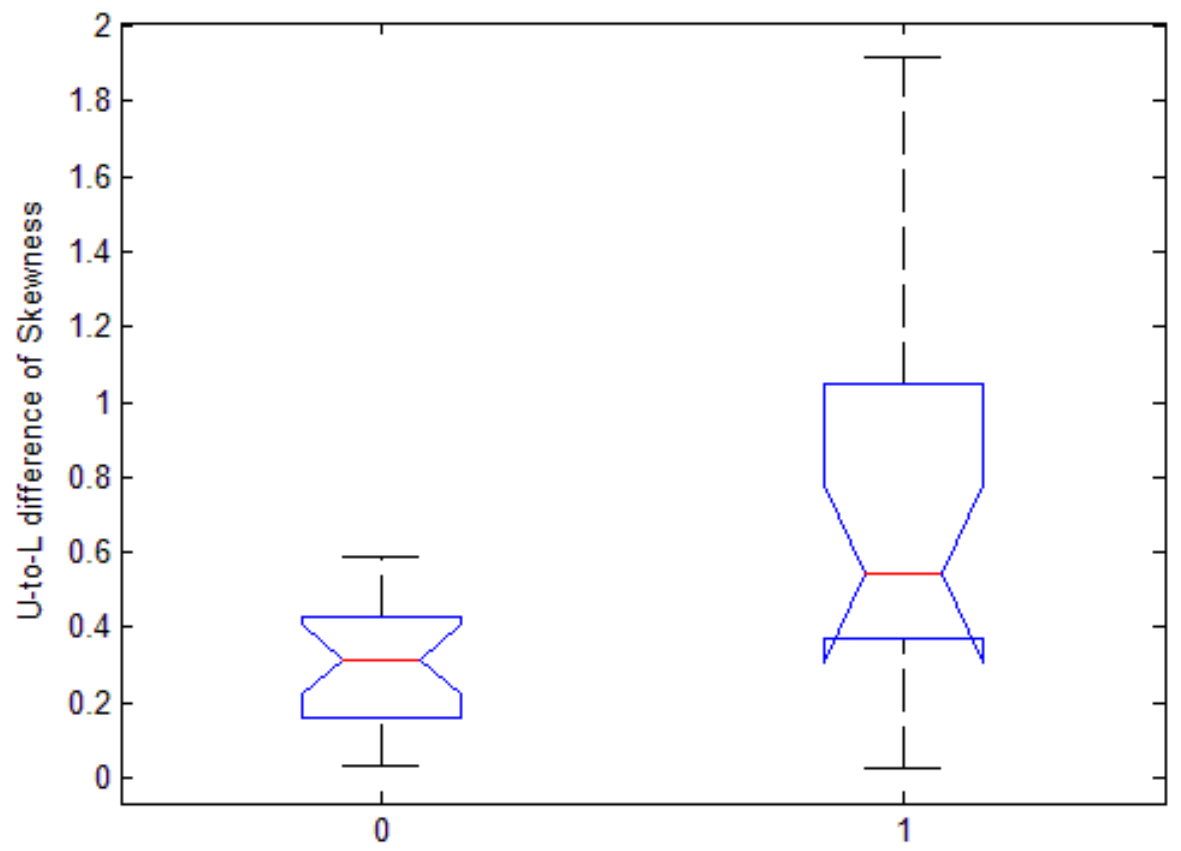

Figure 5-17: Box plot of U-to-L difference of Skewness (0-Normal, 1-NEC) from enhanced thermal images.

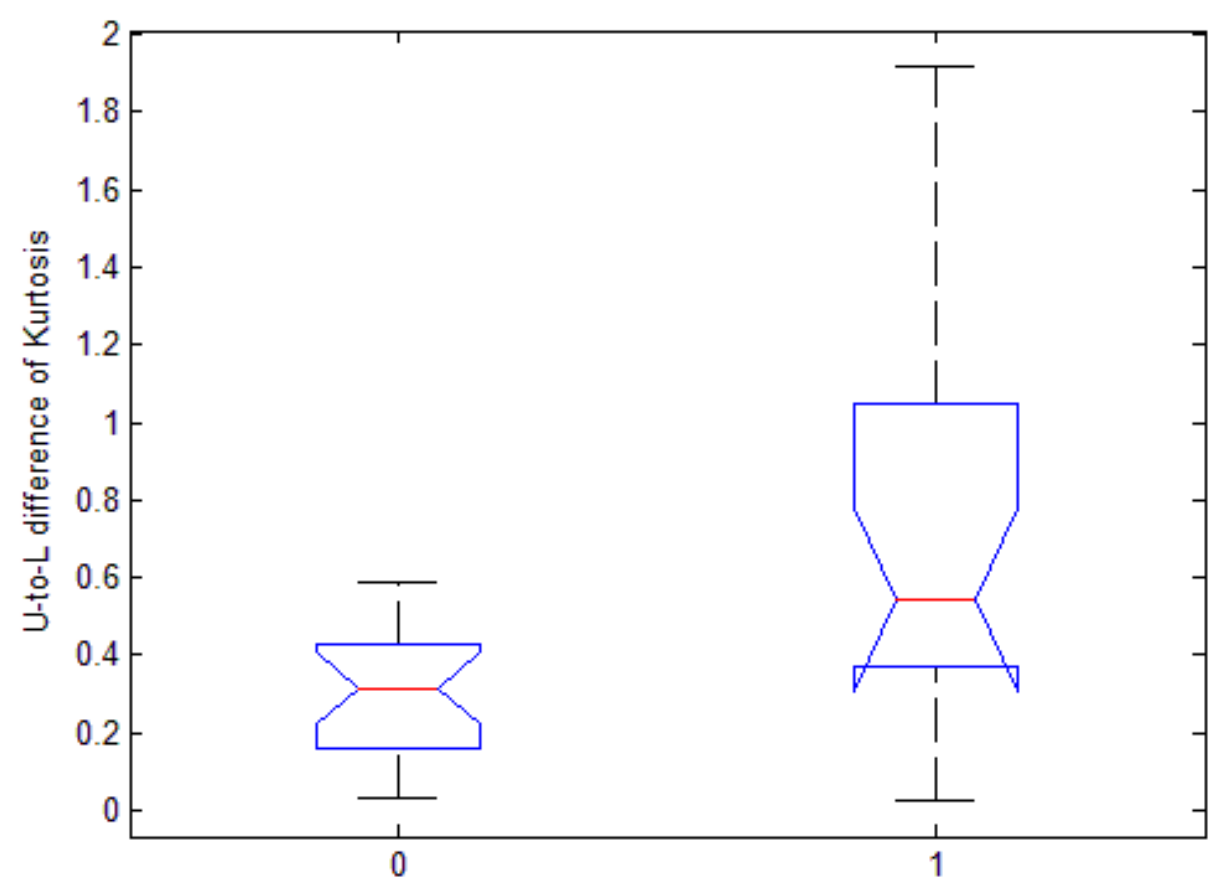

Figure 5-18: Box plot of U-to-L difference of Kurtosis (0-Normal, 1-NEC) from enhanced thermal images. 


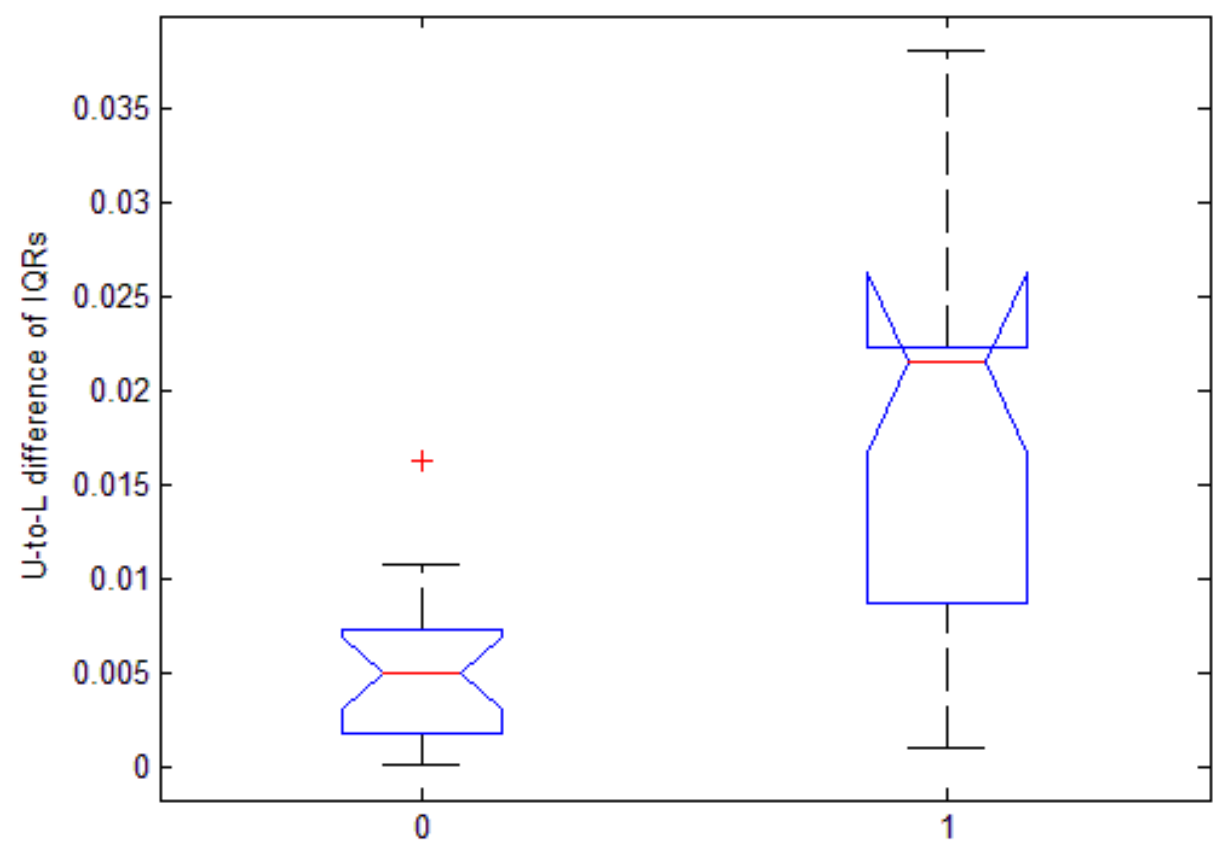

Figure 5-19: Box plot of U-to-L difference of IQRs (0-Normal, 1-NEC) from enhanced thermal images.

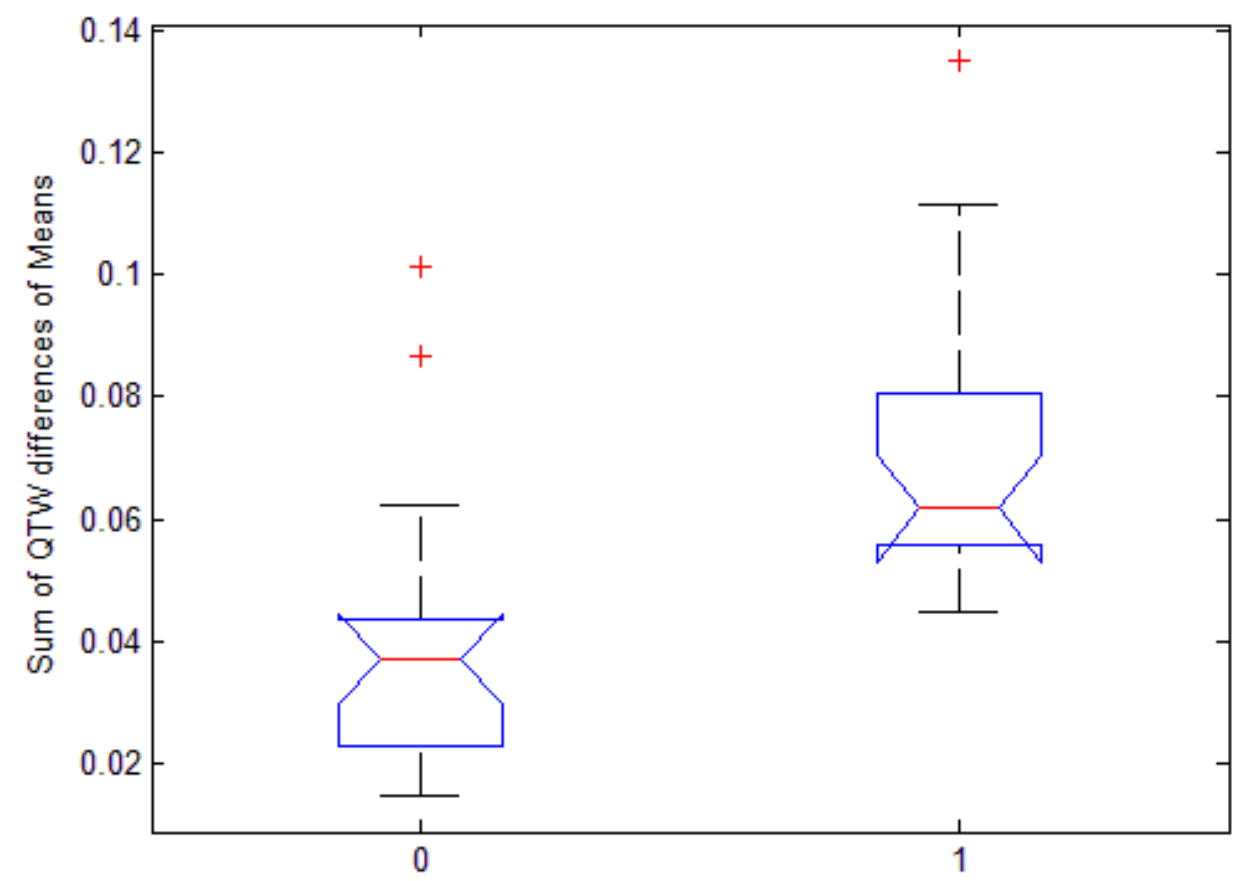

Figure 5-20: Box plot of Sum of QTW differences of Means (0-Normal, 1-NEC) from enhanced thermal images. 


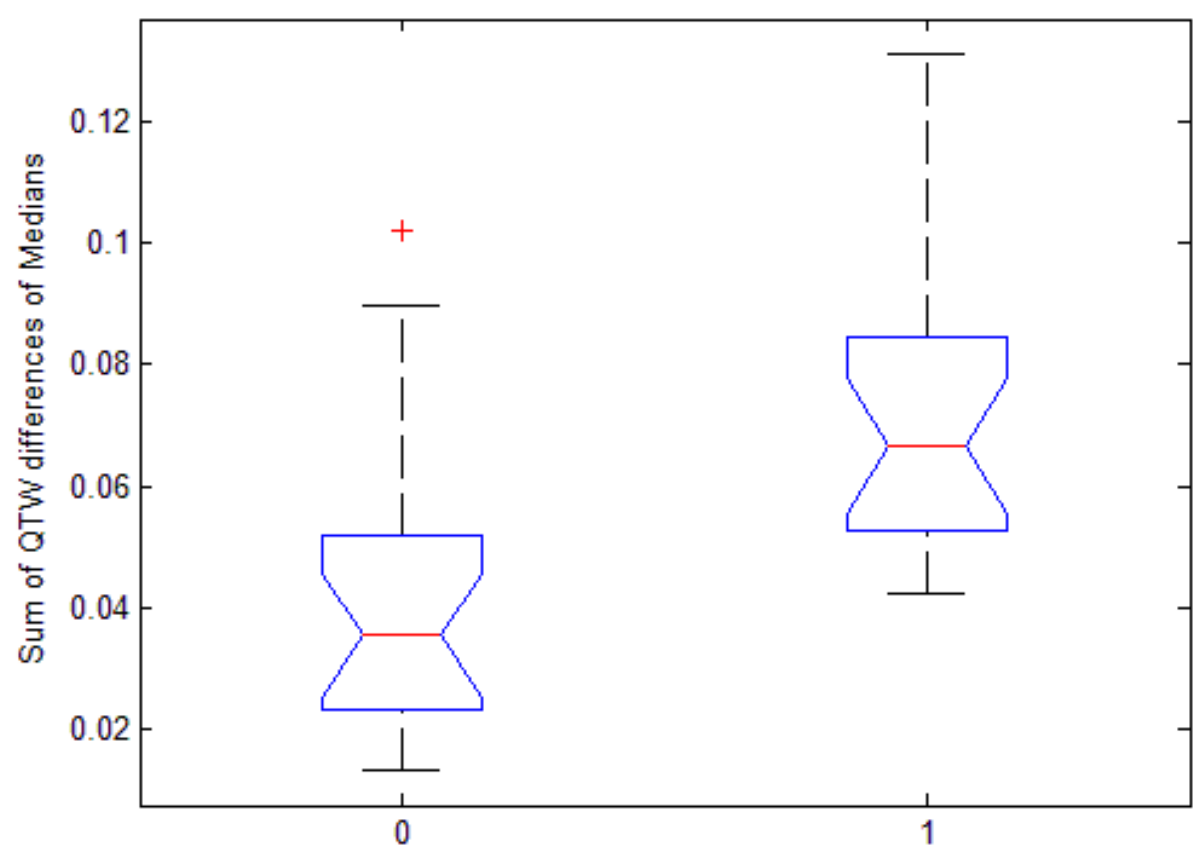

Figure 5-21: Box plot of Sum of QTW differences of Medians (0-Normal, 1-NEC) from enhanced thermal images.

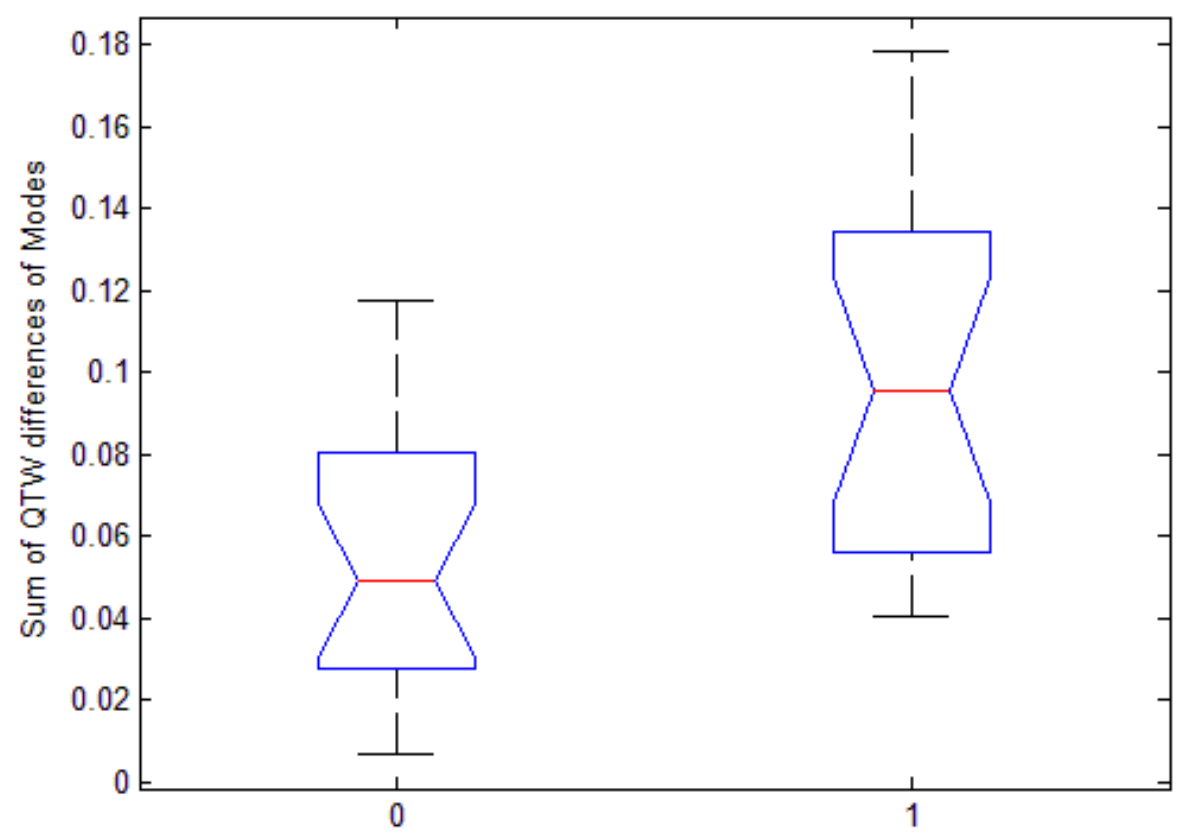

Figure 5-22: Box plot of Sum of QTW differences of Modes (0-Normal, 1-NEC) from enhanced thermal images. 


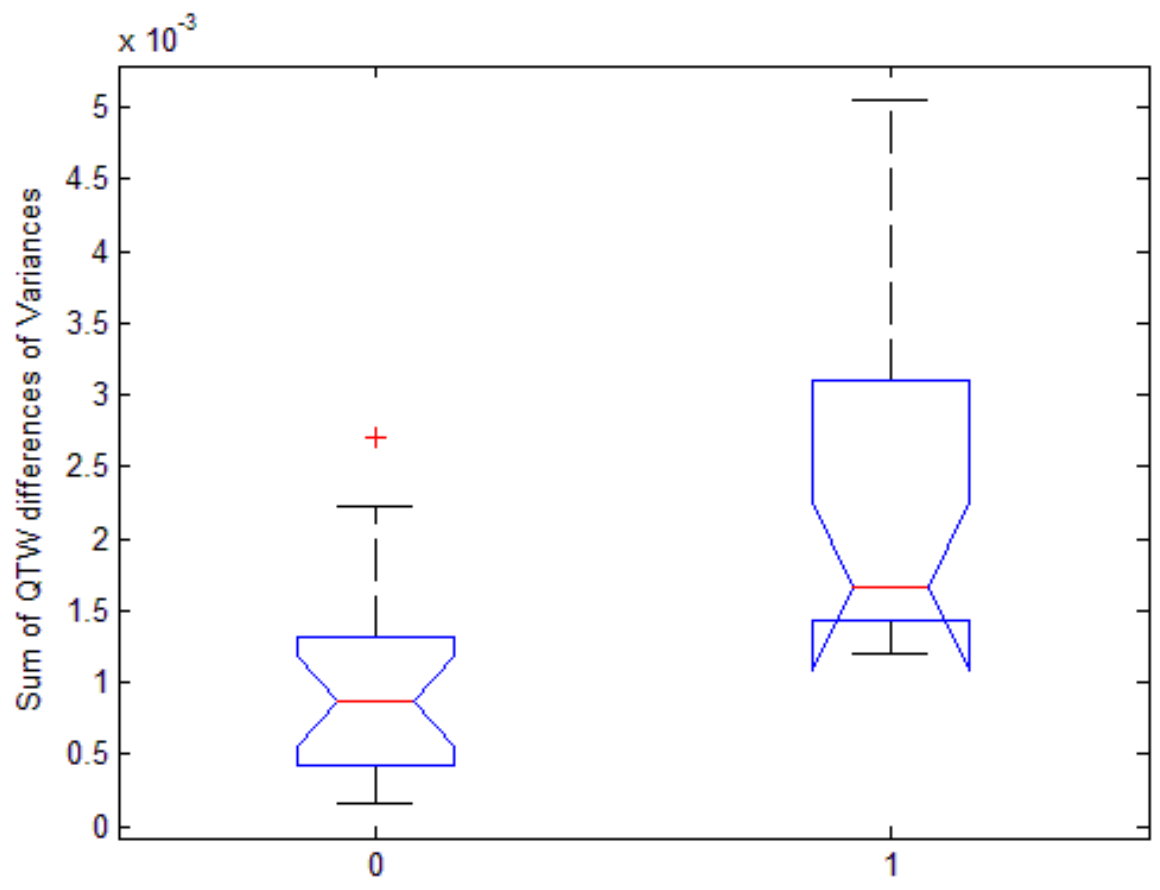

Figure 5-23: Box plot of Sum of QTW differences of Variances (0-Normal, 1-NEC) from enhanced thermal images.

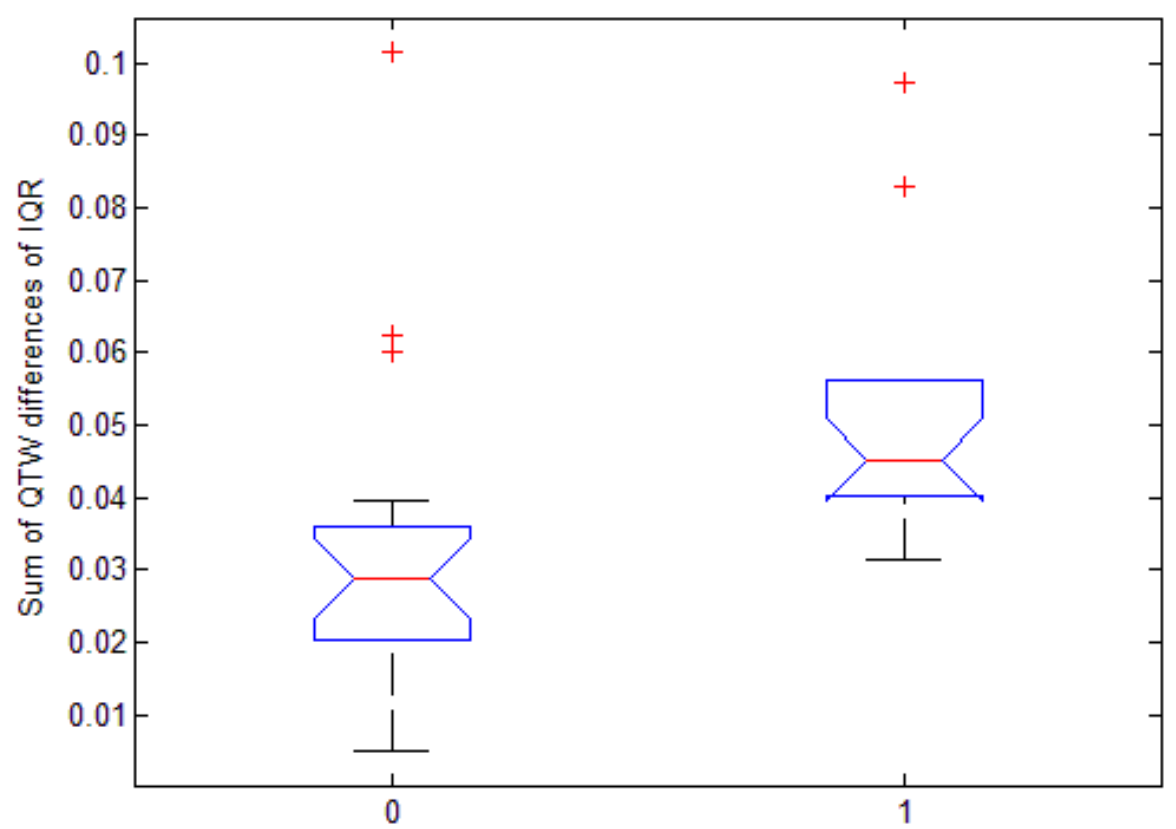

Figure 5-24: Box plot of Sum of QTW differences of IQRs (0-Normal, 1-NEC) from enhanced thermal images. 
Likewise, the Wilcoxon Rank-Sum and Kruskal-Wallis tests were performed for the 27 first order and simple statistical features measured from the GLCMs computed from the original and enhanced images are shown in Table 5-7 and Table 5-8. Results could not be obtained for all median, mode, IQR, and minimum features, since these values were 0 regardless of the group. The null hypothesis could not be reject for the U-to-L means, U-to-L kurtosis, and U-to-L variances, with p-values above 0.87 ; indicating a high degree of uncertainty with this assumption.

Table 5-7: Results of the Wilcoxon Rank-Sum and the Kruskal-Wallis tests performed for all 27 first order and simple statistical features extracted from the GLCMs computed for original thermal images

\begin{tabular}{|c|c|c|c|}
\hline \multirow[b]{2}{*}{ Feature } & \multicolumn{2}{|c|}{ Wilcoxon Rank-Sum } & \multirow{2}{*}{$\begin{array}{l}\text { Kruskal-Wallis Test } \\
\text { p-value }\end{array}$} \\
\hline & $\mathrm{p}$-value & h-value & \\
\hline U-to-L Means & 0.868935 & 0 & 0.850418063 \\
\hline L-to-R-Means & 0.671335 & 0 & 0.654232978 \\
\hline QTW-Means & 0.357971 & 0 & 0.345778586 \\
\hline U-to-L-Medians & $\mathrm{NaN}$ & 0 & $\mathrm{NaN}$ \\
\hline L-to-R-Medians & $\mathrm{NaN}$ & 0 & $\mathrm{NaN}$ \\
\hline QTW-Medians & $\mathrm{NaN}$ & 0 & $\mathrm{NaN}$ \\
\hline U-to-L-Mode & $\mathrm{NaN}$ & 0 & $\mathrm{NaN}$ \\
\hline L-to-R-Mode & $\mathrm{NaN}$ & 0 & $\mathrm{NaN}$ \\
\hline QTW-Mode & $\mathrm{NaN}$ & 0 & $\mathrm{NaN}$ \\
\hline U-To-L-Variance & 1 & 0 & 1 \\
\hline L-to-R-Variance & 0.069538 & 0 & 0.065992055 \\
\hline QTW-Variance & 0.211584 & 0 & 0.203091788 \\
\hline U-To-L-Skew & 0.620618 & 0 & 0.604078091 \\
\hline L-to-R-skew & 0.0771 & 0 & 0.073239203 \\
\hline QTW-Skew & 0.194851 & 0 & 0.186857503 \\
\hline U-to-L-kurtosis & 0.943628 & 0 & 0.924886019 \\
\hline L-to-R-Kurtosis & 0.55569 & 0 & 0.539991479 \\
\hline QTW-Kurtosis & 0.832004 & 0 & 0.813663716 \\
\hline U-to-L-IQR & $\mathrm{NaN}$ & 0 & $\mathrm{NaN}$ \\
\hline L-to-R-IQR & $\mathrm{NaN}$ & 0 & $\mathrm{NaN}$ \\
\hline QTW-IQR & $\mathrm{NaN}$ & 0 & $\mathrm{NaN}$ \\
\hline U-to-L-Min & $\mathrm{NaN}$ & 0 & $\mathrm{NaN}$ \\
\hline L-to-R-Min & $\mathrm{NaN}$ & 0 & $\mathrm{NaN}$ \\
\hline QTW-Min & $\mathrm{NaN}$ & 0 & $\mathrm{NaN}$ \\
\hline
\end{tabular}




\begin{tabular}{|l|l|l|r|}
\hline U-to-L-MAX & 0.211584 & 0 & 0.203091788 \\
\hline L-to-R-MAX & 0.085319 & 0 & 0.081124792 \\
\hline QTW-MAX & 0.150495 & 0 & 0.143918314 \\
\hline
\end{tabular}

Table 5-8: Results of the Wilcoxon Rank-Sum and the Kruskal-Wallis tests performed for all 27 first order and simple statistical features extracted from the GLCMs computed for enhanced thermal images

\begin{tabular}{|c|c|c|c|}
\hline \multirow[b]{2}{*}{ Feature } & \multicolumn{2}{|c|}{ Wilcoxon Rank-Sum } & \multirow{2}{*}{$\begin{array}{l}\text { Kruskal-Wallis Test } \\
\text { p-value }\end{array}$} \\
\hline & $\mathrm{p}$-value & h-value & \\
\hline U-to-L Means & 0.868935 & 0 & 0.850418063 \\
\hline L-to-R-Means & 0.671335 & 0 & 0.654232978 \\
\hline QTW--Means & 0.357971 & 0 & 0.345778586 \\
\hline U-to-L-Medians & $\mathrm{NaN}$ & 0 & $\mathrm{NaN}$ \\
\hline L-to-R-Medians & $\mathrm{NaN}$ & 0 & $\mathrm{NaN}$ \\
\hline QTW--Medians & $\mathrm{NaN}$ & 0 & $\mathrm{NaN}$ \\
\hline U-to-L-Mode & $\mathrm{NaN}$ & 0 & $\mathrm{NaN}$ \\
\hline L-to-R-Mode & $\mathrm{NaN}$ & 0 & $\mathrm{NaN}$ \\
\hline QTW-Mode & $\mathrm{NaN}$ & 0 & $\mathrm{NaN}$ \\
\hline U-To-L-Variance & 1 & 0 & 1 \\
\hline L-to-R-Variance & 0.069538 & 0 & 0.065992055 \\
\hline QTW-Variance & 0.211584 & 0 & 0.203091788 \\
\hline U-To-L-Skew & 0.620618 & 0 & 0.604078091 \\
\hline L-to-R-skew & 0.0771 & 0 & 0.073239203 \\
\hline QTW-Skew & 0.194851 & 0 & 0.186857503 \\
\hline U-to-L-kurtosis & 0.943628 & 0 & 0.924886019 \\
\hline L-to-R-Kurtosis & 0.55569 & 0 & 0.539991479 \\
\hline QTW-Kurtosis & 0.832004 & 0 & 0.813663716 \\
\hline U-to-L-IQR & $\mathrm{NaN}$ & 0 & $\mathrm{NaN}$ \\
\hline L-to-R-IQR & $\mathrm{NaN}$ & 0 & $\mathrm{NaN}$ \\
\hline QTW-IQR & $\mathrm{NaN}$ & 0 & $\mathrm{NaN}$ \\
\hline U-to-L-Min & $\mathrm{NaN}$ & 0 & $\mathrm{NaN}$ \\
\hline L-to-R-Min & $\mathrm{NaN}$ & 0 & $\mathrm{NaN}$ \\
\hline QTW-Min & $\mathrm{NaN}$ & 0 & $\mathrm{NaN}$ \\
\hline U-to-L-MAX & 0.211584 & 0 & 0.203091788 \\
\hline L-to-R-MAX & 0.085319 & 0 & 0.081124792 \\
\hline QTW-MAX & 0.150495 & 0 & 0.143918314 \\
\hline
\end{tabular}




\subsection{DisCUSSION}

In this study, assessment of abdominal thermal symmetry resulted in the computation of 9 first order and simple statistical features. To objectively quantify the degree of thermal symmetry: the U-to-L, L-to-R, and sum of QTW differences were computed for each image. In total, 27 first order and simple statistical features were extracted from the original and the enhanced thermal images. The same features were extracted from the GLCMs computed from the original and enhanced thermal images; providing second order thermal signatures. Analysis of this data was performed using the Wilcoxon Rank-Sum and Kruskal-Wallis tests. These tests compared the distribution of values obtained from thermal symmetry analysis of the NEC and normal groups. Recall that the null hypothesis tested here was that the two groups (0-Normal, 1-NEC) have features that have identical continuous distribution with equivalent means.

Of the 27 first order and simple statistical features extracted from the original thermal images, the null hypothesis was not accepted for 9 of the 27 features. These 9 features were limited to U-to-L differences, and sum of QTW differences. They were as follows: QTW variance, U-to-L median and U-to-L mean, QTW mode, QTW IQR, QTW median, U-to-L mode, QTW mean, and U-to-L variance, with respective values of: $0.00135,0.00298,0.00347,0.0054,0.00952,0.0142,0.0184,0.0267$, and 0.0381. Interestingly, the same 27 features extracted from the enhanced thermal images, resulted in the rejection of the null hypothesis for 12 of the 27 features tested. Of these 12 features the following 6 were rejected with p-values less than 0.005: QTW variance, QTW mean, QTW median, U-to-L variance, U-to-L IQR, QTW 
IQR, p- values were: $0.000817,0.00135,0.00255,0.00298,0.00347$, and 0.00347 respectively. The remaining 6 features were the U-to-L mean, U-to-L mode, QTW mode, U-To-L skewness, U-to-L kurtosis, and U-to-L median; p-values ranged from 0.0109 to 0.0427 . In comparison to the analysis of data computed from original thermal images, the null hypothesis could not be rejected for the following: U-to-L IQR, the U-To-L skewness, and the U-to-L kurtosis. The rejection of the null hypothesis for three addition features indicates that enhanced images increased the ability to discriminate between the groups. In addition, box plots of these features have higher minimum, median, and maximum values for the NEC group in comparison to the normal. This further suggests that the NEC group has a higher degree of thermal asymmetry. The effects of image enhancement need to be further explored.

Results obtained from the thermal symmetry analysis of original and enhanced thermal images suggested that the distributions of certain features do not have identical continuous distributions with equivalent medians, when comparing normal and NEC data. A significance level of 0.05 was used. By visual examination of box plots depicting the distribution of these features, it was evident that the NEC values of these features had a wider distribution and containing larger values. This indicated that the degree of abdominal thermal asymmetry within the NEC group was higher than the normal infants, which may be attributed to the presence of inflammation associated with NEC. Results also indicated that the method of image processing adopted in this study was suitable for this application and for the data 
used in this study, as indicated by the rejection of the null hypothesis for three additional features in comparison to the analysis of the original thermal images.

Analysis of the same 27 first order and simple statistical features extracted from the GLCMs (or $2^{\text {nd }}$ order statistics) computed from the original and the enhanced thermal images had very similar results. The null hypothesis could not be rejected for any of the features and the test could not evaluate all median, mode, IQR, and minimum features. Results could not be generated because these values were initially zero before differentiation was performed, due to the predominance of this value in the GLCM. Interestingly, the null hypothesis was accepted for the U-to-L means, U-to-L kurtosis, and U-to-L variances, with p-values above 0.87 , which indicated a high degree of uncertainty with taking this observation as truth. Although the null hypothesis could not be rejected examination of second order statistical features may support the results obtained from first order analysis, if the GLCM were computed and compared for smaller regions of interest (tiles) of the abdomen. 


\section{Chapter 6: Conclusion}

\subsection{FINAL REMARKS}

The thesis evaluated the potential of abdominal infrared imaging as a method of detecting inflammation associated with NEC; a fully integrated system of the computerised techniques described in section 4.6 was used. The development of image enhancement techniques suitable for this medical imaging application was also attempted. Thermal symmetry analysis defined in section 4.5 Image Analysis, computed the U-to-L, L-to-R, and sum of QTW differences of 9 first and second ordered statistical features. The Wilcoxon Rank-Sum and Kruskal-Wallis tests were performed to compare the distributions of these 27 first order and 27 second order statistical features extracted of the two groups (0-Normal and 1-NEC). This was performed for both the original and the enhanced abdominal thermal images.

The distribution of 9 out of the 27 first order statistical features obtained from thermal symmetry analysis of original images were determined not to have identical continuous distribution with equal medians when comparing the two groups (0Normal and 1-NEC). These features and their respective p-values were: QTW variance (0.00135), U-to-L median (0.00298), U-to-L mean (0.00347), QTW mode (0.0054), QTW IQR (0.00952), QTW median (0.0142), U-to-L mode (0.0184), QTW mean (0.0267), and U-to-L variance (0.0381). First order thermal symmetry analysis repeated for images enhanced using the techniques described in section 5.1 Image Enhancement; 12 features were deemed to have statistically different distribution of values, in a comparison of the two groups. The features and their p-values were: 
QTW variance (0.000817), QTW mean (0.00135), QTW median (0.00255), U-to-L variance (0.00298), U-to-L IQR (0.00347), QTW IQR (0.00347), U-to-L mean (0.0109), U-to-L mode (0.0301), QTW mode (0.0301), U-to-L skewness (0.0301), Uto-L kurtosis (0.0301), and U-to-L median (0.0427). The rejection of the null hypothesis for three addition features indicated that enhanced images may increase the ability to discriminate between the groups, this requires further investigation. For both the original and the enhanced thermal images, the 9 and 12 first order statistical features highlighted had a wider distribution and larger values for the NEC group compared to the normal group. For the data set used, results suggested that the NEC group had a higher degree of thermal asymmetry, in comparison to the normal group. This may indicate the presence of inflammation characteristic of NEC.

The same statistical tests were repeated to compare the distributions of the 27 second order statistical features extracted from the NEC and normal groups. Similar results were obtained for both the original and enhanced thermal images. The results were unanimous; the null hypotheses could not be rejected with a higher degree of uncertainty for most features, or could not be determined. Although, a high degree of uncertainty surrounded the acceptance of the null hypothesis for Uto-L means, U-to-L kurtosis, and U-to-L variances, which had p-values above 0.87 . Analysis of median, mode, IQR, and minimum features were not possible due to the domination of the zero value in the GLCM.

\subsection{CONTRIBUTIONS TO KNOWLEDGE}

In addressing the problem defined in this thesis three major contributions to knowledge have been accomplished. 
Firstly, the objective quantification of abdominal thermal symmetry, to assess the potential of IR imaging as a method of NEC detection was performed. To achieve this, the tasks of developing appropriate ROI segmentation techniques and measurement of abdominal thermal symmetry was addressed. The methods adopted in this thesis are described in "Infrared Imaging and Classification of Neonates with Necrotizing Enterocolitis," published as part of the proceedings of the 2012 World Congress on Medical Physics and Biomedical Engineering [94]. Results suggested that the first order statistics computed for this study were suitable in identifying a higher degree abdominal thermal asymmetry for the NEC group. In particular results of U-to-L and sum of QTW differences of the: mean, median, mode, variance, IQR, skewness and kurtosis. This was especially true of the QTW variances, which achieved the lowest p-values.

Secondly, the development of image enhancement techniques suitable for unveiling hidden details in medical thermal images was achieved; this technique was described in "Image processing of infrared thermal images for the detection of necrotizing enterocolitis" published in the conference proceeding of SPIE Medical Imaging 2013 [93].

Lastly, to our knowledge this thesis was the first attempt to fully integrate into a system a set of comprehensive computerised techniques for the assessment of abdominal thermal imaging as a potential method of NEC detection. 


\subsection{FUTURE WORK}

Results of the first order thermal symmetry analysis suggests that abdominal thermal imaging may be a potential avenue for the detection of inflammation associated with NEC. For this reason, the next phase should expand this research to become a multi-centered prospective study. This is vital for the collection of more images and confirmation of the findings of this work. The goal here is to make inferences about a larger population of NEC and normal infants and strengthen comparisons. As well, the effects of the image enhancement technique adopted needs to be further confirmed, and investigated using a larger data set. A statistical comparison of the distribution of the second order features could not differentiate between the two groups. Although, extracting these features from smaller ROIs (of "tiles") of the abdomen than those used in this work may produce different results. The development of automated registration and segmentation techniques to increase the level of clinical efficiency should be explored. In addition, the dynamic assessment of abdominal infrared images may reveal thermal abnormalities suggested by abnormal temporal evolution of skin temperature and should be examined. 


\section{Appendices}

\section{APPENDIX A}

The document in Appendix A. is the CHEO REB Research Ethics Proposal submitted in August 2006 [103]. The last page of this document is the consent form used in this study. Appendix A.2 CHEO Parent Information Sheet contains "The CHEO Parent

Information Sheet," which was given to parents to review prior to enrolling their infants in the study [104]. 
\begin{tabular}{l}
\hline TITLE OF RESEARCH STUDY: Prospective Assessment of Necrotizing Enterocolitis with \\
Medical Thermography
\end{tabular}

SPONSORING AGENCY:

(if industry sponsored, a fee of $\$ 1,500$ will be charged for the review of the proposal)

\section{PRINCIPAL INVESTIGATOR}

\begin{tabular}{|l|l|l|l|}
\hline \hline NAME & DEPARTMENT/PSU & EMAIL ADDRESS & PHONE NUMBER \\
\hline Monique Frize & $\begin{array}{l}\text { Professor at Carleton } \\
\text { University } \\
\text { Department of Systems and } \\
\text { Computer Engineering }\end{array}$ & $\underline{\text { mfrize@connect.carleton.c }}$ & XXX-XXX-XXXX \\
\hline
\end{tabular}

Signature:

This signature confirms that, as principal investigator, you assume full responsibility for the research as outlined in this application and that you will notify the REB of any developments in the project including an annual report, reports of adverse events, reports of subject recruitment, reports of any study amendments, and a study termination report. The principal investigator must also maintain copies of all pertinent information related to the research activities in this project, including copies of the informed consent agreements obtained from all participants. The REB should also be notified if one of the principal investigators leaves this institution or the project. Finally, the REB must be notified of the relationship between the Sponsor and Principal Investigator(s) changes in any way (See Item 2, page 10).

\section{CO-INVESTIGATORS}

\begin{tabular}{|c|c|c|c|}
\hline NAME & AFFILIATION* & EMAIL ADDRESS & PHONE NUMBER \\
\hline 1. Robin Walker & $\begin{array}{l}\text { Vice-President Medicine } \\
\text { IWK Health Centre } \\
\text { Halifax }\end{array}$ & walkcr@iwk.nshealth.ca & XXX-XXX-XXXX \\
\hline \multicolumn{4}{|l|}{ Signature: } \\
\hline $\begin{array}{l}\text { 2. Christophe } \\
\text { Herry }\end{array}$ & $\begin{array}{l}\text { PhD student } \\
\text { Carleton University }\end{array}$ & cherry@sce.carleton.ca & $\begin{array}{l}\text { XXX-XXX-XXXX } \\
\text { ext. XXXX }\end{array}$ \\
\hline \multicolumn{4}{|l|}{ Signature: } \\
\hline 3. Erika Bariciak & $\begin{array}{l}\text { Neonatologist } \\
\text { CHEO }\end{array}$ & bariciak@cheo.on.ca & $\begin{array}{l}\text { XXX-XXX-XXXX } \\
\text { ext. XXXX }\end{array}$ \\
\hline \multicolumn{4}{|l|}{ Signature: } \\
\hline
\end{tabular}


SIGNATURES: All signatures must be obtained prior to submission to the REB. This includes all personnel directly or indirectly involved in the study (i.e. Laboratory, Nursing, Allied Health). The Director of Pharmacy must approve all studies involving drugs.

\begin{tabular}{|c|c|c|c|c|}
\hline Position & Print Name & Signature & Phone Number & Date \\
\hline $\begin{array}{l}\text { Operations } \\
\text { Director }\end{array}$ & & & & \\
\hline $\begin{array}{l}\text { Department } \\
\text { Chief } \\
\text { (as } \\
\text { applicable) }\end{array}$ & & & & \\
\hline $\begin{array}{l}\text { Professional } \\
\text { Practice } \\
\text { Leader } \\
\text { (as } \\
\text { applicable) }\end{array}$ & & & & \\
\hline $\begin{array}{c}\text { Chief } \\
\text { Nursing } \\
\text { Officer } \\
\text { (as } \\
\text { applicable) }\end{array}$ & & & & \\
\hline $\begin{array}{l}\text { Director of } \\
\text { Pharmacy } \\
\text { Or } \\
\text { Delegate } \\
\text { (as } \\
\text { applicable) }\end{array}$ & & & & \\
\hline
\end{tabular}

My signature attests to the following:

s proposal and to the best of my abilities.

- I am satisfied that the objectives, design, methods, and contributions of the proposed research have sufficient scientific validity, quality and merit to warrant the implementation of this project.

- The investigator is in good standing at the Children's Hospital of Eastern Ontario or CHEO Research Institute.

- The investigator has the credentials/expertise to conduct the research being proposed in this application.

- My Department or Service has the resources (e.g.; space, personnel and patient population) to support this research. 
STUDY INFORMATION: Please answer ALL of the following questions and provide additional information as applicable:

1. Does the research involve the use of a pharmaceutical agent?

All clinical trials must be approved be Health Canada prior to the initiation of the trial.

Please refer to the TPP website: www.hc-sc.gc.ca/hpb-dgps/therapeut for further information. Guidance documents can be obtained on this same website with the following attachment ..../gtnkebg/draft_guide_industry. Html

2. Conflict of Interest:

Does the principal investigator(s) or any co-investigators involved in this research study:

(a) Function as an advisor, employee, officer, director or consultant for the sponsor?

(b) Have direct or indirect financial interest in the drug, device or technology employed (including patents or stocks) in this research study?

(c) Receive an honorarium or other benefits from the sponsor (apart from fees for service)?

(d) If the answer is YES to any of the above, please describe and explain how that conflict is being managed to ensure that participants rights and welfare are not affected.

3. Sponsor Agreement

(a) Will there be a signed contract agreement with the sponsor related to this study?

(b) If YES, will it in any way limit your access to the research data, or limit your right to publish the study results? Please explain.

Note: Agreements must be reviewed and signed by authorized institutional officials.

4. Is the risk of harm or discomfort anticipated in the research no greater than those ordinarily encountered in daily life or during the performance of routine physical or psychological examinations or tests (i.e. chart reviews)?

If YES, the study may be eligible for expedited review in which the Chair alone or a small subcommittee reviews the proposal. 


\title{
Application to the Research Ethics Board of the Children's Hospital of Eastern Ontario
}

\author{
Title of research study: Prospective assessment of necrotizing enterocolitis with \\ medical thermography
}

\section{Synopsis:}

In this study we propose to investigate the use of medical thermography for the detection of inflammation and pain associated with necrotizing enterocolitis (NEC).

NEC is a serious condition that affects primarily premature newborns. It manifests itself with a wide range of symptoms and rapid deterioration often culminating in septic shock. The affected bowel exhibits areas of inflammation, necrosis and often perforation. These may be confined to a single area, be found in many disconnected areas or involve large continuous segments of small or large bowel up to the full length of the bowel. It is not clear what causes NEC but it may involve a combination of injury to the bowel, bacterial invasion of the bowel wall and presence of feedings in the bowel.

There are no definite methods of prevention. Therapy is initially supportive including cessation of feedings, provision of intravenous nutrition, antibiotics and treatment of shock. Perforation or ongoing deterioration may require surgical intervention.

Mortality has improved in recent years but is still about $15 \%$ and survivors may suffer significant complications including long-term damage to bowel function.

Because of the massive inflammation of bowel and the intense reaction of adjacent peritoneum, this condition produces significant pain and tenderness. Perforation of the bowel further leads to peritonis, a condition known in adults to be extremely painful. As newborns are believed to feel pain more intensely than older persons because of immaturity of protective mechanisms, recognition and treatment of pain is very important in this population.

Medical thermography is a non-invasive, non-radiative imaging procedure that records the thermal distribution of human body by use of an infrared camera. The thermal distribution of a person is closely related to physiological processes and can provide valuable information about the initiation and progression of functional abnormalities or pathologies, such as the inflammatory and painful processes involved in NEC.

This study consists of two phases. First we will consider a population of premature newborns without any signs of NEC in order to derive a standard thermal distribution for healthy newborns. Then, we propose to study a population of premature newborns with clinical signs of NEC, at various stages of the disease. From the analysis of the two populations, we will devise a decision-support system that could help the physician make informed decisions about the diagnosis and management of affected newborns. 


\section{Summary of Ethical Considerations:}

In this study, we will be taking several infrared images of the abdomen of the subject. Medical Thermography is non-invasive, non-ionizing (cf. page 7-8). It merely records the infrared radiation emitted naturally by all bodies. There are no known risks directly associated with thermography (cf. page 8-9).

Medical thermography requires a period of slight cooling of the subject in order to stabilize the body surface temperature. The room temperature will be maintained slightly below thermoneutrality, as is usually the case in a neonatal intensive care unit, to ensure accurate imaging without inducing any sustained cold stress.

The hood of the incubator will be lifted up and the mattress on which the subject lies will be pulled out for a brief period of time (no more than 1 minute), to allow for the infrared radiation naturally emitted by the subject's body to be recorded by the infrared camera. This step is necessary since infrared radiation does not pass through the Plexiglas walls of an incubator and the infant's skin needs to cool off slightly. If the infant is being cared for on an overbed warmer, the heater will be turned off for a brief period (no more than 1 minute). This step is necessary as the heat being emitted by the warmer may interfere with the recording of infrared radiation being emitted by the baby. All clothing, if any, will be removed except for clothes covering extremities such as the head, hands or feet, since they will not affect the thermal distribution over the abdomen. Several images of the subject will be taken. Then the mattress will be pushed back into the incubator and the hood will be closed, or the overbed heater will be turned back on. The brief period of time during which the subject is outside of the incubator will not exceed one minute. Necessary precautions will be taken to ensure that the decrease of the subject's body temperature during the imaging session does not exceed acceptable limits. The brief removal of the subject from the incubator may also increase the subjects' stress level. However, since the session is very short, it will not affect the subject more than for regular daily procedures. Neonatal health care staff will be present at all times to ensure that the subject's vital signs are within acceptable limits. Any degradation of a subject's vital signs will result in immediate stopping of the imaging procedure (cf. pages 9-10). This process will be repeated up to three times, over the typical timeframe of evolution of necrotizing enterocolitis.

There are no immediate medical benefits for the subjects involved in this study, as this is an investigative study to determine the possible value of thermography in the assessment and management of necrotizing enterocolitis (NEC) (cf. page 9). However, being able to better understand the physiological mechanisms associated with NEC could lead to improved management of the disease and reduce the mortality and morbidity rates in the future. Pain often results from inflammation, which releases heat. Early research by our team suggests that painful disease processes are often visible in thermographic images. Recognition of pain is a problem in newborns, particularly those at very early gestations. Our proposed study will attempt firstly to correlate a disease process well-known to cause pain (NEC) with thermographic images as a first step towards possible other studies of early diagnosis of pain in newborns. If we are able to demonstrate that thermography can 'see' pain, this would provide an objective means of assessing pain and titrating treatment to degree of pain, something not currently available. 
Dr. Erika Bariciak will identify the subjects who fit the criteria for inclusion in our study and will approach the parents with a preliminary outline of the study and its purpose only. An investigator who does not have any relation to the subject will then approach the potential subject's parents and give all the information necessary for the parents to make an informed decision whether or not to participate in this study. No information concerning the subject will be collected unless the parents expressly agree to it. A sample informed consent form is provided on page 15.

If the subject's parents decide to participate in the study, some information will be collected for each imaging session including the age, sex and temperature (cf. page 11). The images will be stored on a personal computer for analysis. The analysis will begin when all the images for all subjects are available.

The proposed starting date for the recording of the thermal images is September 2006. The data collection for the control infants is expected to be completed by September 2007. Final results are expected in December 2007.

We would like to obtain a population of 30 to 40 infants for the control group as well as for the group suspected of being affected by NEC. A sample size of 30 to 40 infants would ensure a statistical power of $95 \%$ and a type I error less than 5\%, when comparing the temperature distributions of the two populations.

For the first part of the study (control population of 'healthy babies'), the subjects must meet the following criteria in order to be considered:

1. Their number of completed weeks of gestation at birth is less than or equal to 32 weeks

2. They do not exhibit any clinical, radiological, pathological signs commonly associated with necrotizing enterocolitis.

3. They are not affected by any other pathological conditions that could alter their thermal regulation.

4. They are in stable condition.

For the second phase of the study (population of babies with or suspected to have NEC), the subjects must meet the following criteria:

1. Their number of completed weeks of gestation at birth is less than or equal to 32 weeks

2. They exhibit clinical, radiological, pathological signs commonly associated with necrotizing enterocolitis OR they are at high risk of developing necrotizing enterocolitis OR they are suspected to begin developing necrotizing enterocolitis.

3. They are in stable condition.

The population at risk of NEC is the early preterm infant. It is therefore necessary that the control and NEC groups be in the gestational range for NEC risk (in our study, less than or equal to 32 weeks). The first phase will be our control group of infants without any suspicion of NEC or abdominal pathology. The second phase will be infants with signs of or at risk for NEC. 


\section{Introduction}

It is now well known that thermoregulation of the human body is affected by a wide range of factors including pathological abnormalities. The recording of temperature distribution of human body can therefore provide valuable information about the underlying physiological processes that cause those abnormalities. Human skin plays a major role in thermoregulation by dissipating or preserving heat. The dissipation of heat through the skin is mainly radiative and occurs in the infrared part of the spectrum, which makes infrared detectors particularly suitable for the recording of skin temperature distribution, and by extension of whole body core temperature distribution. Clinical infrared thermography or infrared thermal imaging is defined as the recording of the temperature distribution of human body using infrared radiation emitted by the surface of that body i.e. the skin. Many studies have been carried out on the assessment of thermal imaging for various medical applications since its introduction in the early fifties.

Many attempts to quantify the analysis of thermal images were proposed in the literature. Among the various applications of medical infrared thermal imaging, oncology and particularly breast cancer detection has been the most thoroughly explored and is still under review by many recent studies. Pain management and assessment is another prolific area of application of thermography.

The processing of thermal images relies mostly on the assumption that the temperature distribution of normal healthy people is symmetrical from a contralateral point of view, which has been confirmed by many clinical studies (ref. [3][4]). However, some pathologies may exhibit a contralateral symmetry in the temperature distribution of the body and it is therefore necessary to complement this approach by a more thorough search for hotter or colder areas.

In previous work, we investigated the computerized assessment of thermal images of pain patients (ref. [1][2]). The data available came from hundreds of digital thermal infrared images of pain patients, collected by Professor Monique Frize and her team at the Pain Clinic of the Moncton Hospital. A second set of images was taken on normal healthy volunteers at Carleton University and served as our control population. The results from the analysis gave very promising results on a database of 24 images, when compared with the actual outcome determined by an experienced neurosurgeon Dr. Quartey, Moncton Hospital. We successfully applied similar processing techniques to thermal images of breast cancer.

Necrotizing enterocolitis involves inflammation of the colon of premature infants and is believed to be extremely painful. Recognition of pain is a problem in newborns, particularly those at very early gestations. Currently, only abdominal x-ray imaging is used systematically to diagnose NEC. However, diagnosis is not always easy, particularly if $\mathrm{x}$-rays show no signs of the disease. In addition, the inflammation and pain felt by affected babies are present prior to the appearance of significant signs on the x-ray images. Our proposed study will attempt firstly to correlate a disease process well-known to cause pain (NEC) with thermographic images as a first step towards possible other studies of early diagnosis of pain in newborns. If we are able to demonstrate that thermography can 'see' pain, this would provide an objective means of assessing pain and titrating treatment to degree of pain, something not currently available. 


\section{Objectives:}

1. Derive the temperature distribution of the abdomen of normal healthy babies, whose number of completed weeks of gestation at birth is less than or equal to 32 weeks, using medical thermography.

2. Assess the potential of medical thermography in helping to determine the presence and levels of pain and inflammation in babies affected (or suspected to be affected) by necrotizing enterocolitis.

\section{Ethical Considerations:}

\section{a) Ethical procedures used in the conduct of research (details of subject involvement)}

In this study, we will be taking several infrared images of the abdomen of the subject. Medical thermography requires a period of slight cooling of the subject in order to stabilize the body surface temperature. In the case of adults, this is achieved by placing the subject in a room whose temperature is maintained at around 20 degrees Celsius with variations of no more than 0.1 degrees Celsius. The room temperature should not be uncomfortable for the subject, and in particular, should not induce shivering. In the case of newborns, for which thermoneutrality is at a higher temperature, the room temperature will be maintained slightly below thermoneutrality, as is usually the case in a neonatal intensive care unit, to ensure accurate imaging without inducing any sustained cold stress.

The imaging procedure will be as follows:

- Clothing covering the abdomen of the baby, if any, will be removed. This will be done inside the incubator.

- The hood of the incubator will be lifted up and the mattress on which the baby lies will be gently rolled out for $45-60$ seconds, to allow for the infrared radiation naturally emitted by the baby's body to be recorded by the infrared camera. This step is necessary since infrared radiation does not pass through the Plexiglas walls of an incubator. If the infant is lying on an overbed warmer, the heater will be shut off for 45-60 seconds. After a few seconds of stabilization, several images of the baby will be taken. The baby will be maintained still by a nurse during the actual recording of each image (which is essentially instantaneous) to ensure that the image is properly recorded.

- Then the mattress will be pushed back into the incubator and the hood will be closed, or the overbed warmer will be turned on again. A nurse will put the baby's clothes back on.

- The overall process outside the incubator/with the heater turned off should take approximately a minute. Nurses will assess the pain level of the babies during the imaging procedure (using the standard NICU tool: PIPP.) 
Real-time procedure:

Time 0 minutes: Procedure commences: clothing removed except for clothes covering extremities such as the head, hands or feet; this is done inside incubator/with the overbed warmer turned on. [ $\sim 1$ minute]

Time 1 minute: Incubator hood lifted and mattress pulled out/overbed warmer turned off. Infrared radiation naturally emitted by the subject's body recorded by infrared camera. Several images of the subject will be taken; each is instantaneous but baby will be gently held still by nurse for the moment of each image recording (as is usual practice for all imaging procedures, e.g., X-ray imaging.) $[\leq 1$ minute $]$

Time 2 minutes: Mattress pushed back into the incubator and hood closed/overbed warmer turned on. Clothing replaced [ $\sim 1$ minute]

Time 3 minutes: Procedure concludes

Total procedure time $=\sim 3$ minutes

Time outside incubator $=\leq 1$ minute

This process will be repeated up to three times, over the typical timeframe of evolution of necrotizing enterocolitis.

There would be no more than one procedure per 24 hours period. For babies with NEC, evolution of the disease usually progresses rapidly, often over as little as 24 hours. Therefore it will be important to be able to image on a daily basis during this period, provided the baby's condition is stable enough to allow imaging. However, the limit to one daily procedure is intended to ensure that there can be no deleterious effect from this procedure, even though it is non-invasive and requires minimal handling. The limitation could be later reduced if the technique proves as easy and affects babies' condition as little as expected.

All the tests will be performed at the CHEO.

\section{b) Known or possible risks and benefits to subjects}

There are no known risks associated with thermography. This imaging modality is non-invasive, non-ionizing. It does not send any electromagnetic waves to the subject. It merely records the infrared radiation emitted naturally by all bodies.

However, the population considered in this study is typically cared for in an incubator or on an overbed warmer. Plexiglas walls do not let infrared radiation pass, therefore it is necessary to remove the subjects from the incubator. The heat being emitted by the overbed warmer may interfere with the recording of infrared radiation emitted from the baby, thus the warmer will be turned off while the images are being recorded. As mentioned in the previous paragraph, the overall process should not exceed one minute. The brief period during which the subject is outside of the protective environment of an incubator or overbed warmer could induce a decrease of the subject's body temperature if appropriate precautions are not taken. The brief removal of the subject from the incubator may also increase the subjects' stress level but no more than during regular medical care. Handling may cause transient changes in heart rate, blood oxygen saturation or blood pressure but there is no evidence that 
these changes are significant or harmful during brief gentle handling in stable babies. As noted below, the procedure will not be performed or will be immediately discontinued in any baby who manifests any sign/s of instability as defined below.

There are no immediate medical benefits for the subjects involved in this study, as this is an investigative study to determine the possible value of thermography in the assessment and management of necrotizing enterocolitis (NEC). However, being able to better understand the physiological mechanisms associated with NEC could lead to improved management of the disease and reduce the mortality and morbidity rates in the future.

\section{c) Precautions taken to deal with known or possible risks}

Neonatal health care specialists will be present at all times to ensure that the subject's vital signs are within acceptable limits. Any degradation of a subject's vital signs will result in immediate stopping of the imaging procedure and the health care staff present will attend the patient at the time.

The investigators will in-service the nursing staff prior to the study. NICU nurses are familiar with signs of instability but as a precaution a list of specific changes that require discontinuation of the procedure will be drawn up, to include particularly changes in respiratory status, heart rate, blood pressure, colour, and $\mathrm{O} 2$ saturation. In addition, the nurse's decision to discontinue for any other reason, including her/his perception that the baby is not tolerating the procedure, will be final.

Changes that would be considered significant are either of the following:

- increase in respiratory rate to $60 \mathrm{bpm}$ or more

- increase of $20 \mathrm{bpm}$ or more from baseline if over $60 \mathrm{bpm}$

- any apnea or increased periodicity of breathing

- decrease in heart rate below $100 / \mathrm{min}$ or greater than $180 / \mathrm{min}$ or change of $30 / \mathrm{min}$ or more from baseline

- increase or decrease in blood pressure of $10 \mathrm{~mm} \mathrm{Hg}$ or more

- any appearance of cyanosis, peripheral or central

- O2 saturation falling to less than $88 \%$ or decrease from baseline of $5 \%$ or more.

- Increase in PIPP score to $>6$

\section{d) Investigator's relation to subjects}

The principal investigator does not have any relation to the subjects. One of the coinvestigators (Dr. Erika Bariciak) is a neonatologist at the CHEO and therefore may have a physician/patient relationship with some of the subjects. Neither Dr Bariciak, therefore, nor the any subject's attending physician will be responsible for recruitment of the subject into the trial.

\section{e) Presentation of Consent form}


If the parents of a potential subject show interest in the study after being approached by one of the investigators, a package including the description of the study and the consent form will be presented to the subject's parents. They will be given time to carefully review the consent form and ask any questions they might have. (See below re: recruitment procedures.)

\section{f) Part I: Healthy babies (control group)}

Number of subjects

Approximately 30 to 40 infants. The infants will be drawn solely from CHEO.

\section{Inclusion criteria}

("they" or "their" refers to the subjects)

1. Their number of completed weeks of gestation at birth is less than or equal to 32 weeks

2. They do not exhibit any clinical, radiological, pathological signs commonly associated with necrotizing enterocolitis.

3. They are not affected by any other pathological conditions that could alter their thermal regulation.

4. They are in stable condition.

\section{g) Part II: Babies confirmed or suspected to have NEC}

\section{Number of subjects}

Approximately 30 to 40 infants. The infants will be drawn solely from the CHEO.

\section{Inclusion criteria for the second part of the study:}

1. Their number of completed weeks of gestation at birth is less than or equal to 32 weeks

2. They exhibit clinical, radiological, pathological signs commonly associated with necrotizing enterocolitis OR they are at high risk of developing necrotizing enterocolitis OR they are suspected to begin developing necrotizing enterocolitis.

3. They are in stable condition (with respect to respiratory status, heart rate, blood pressure, oxygen saturation and pain control.)

\section{h) Rationale for adequacy of subject sample size}

A sample size of 30 to 40 infants will ensure a statistical power of $95 \%$ and a type I error less than $5 \%$, when comparing the temperature distributions of the two populations. 


\section{i) Subject recruitment procedures, information to be collected and sources of information}

Dr. Erika Bariciak will identify the subjects who fit the criteria for inclusion in our study and will approach the parents with a preliminary outline of the study and its purpose only; Dr Bariciak and any person not at arms length from the clinical care of the infants will not otherwise be involved in subject recruitment. If the parents indicate to Dr Bariciak that they are not interested in receiving any further information about the study no further attempt to recruit the subject will be made. If the parents indicate willingness to see/hear further information, an investigator who does not have any relation to the subject will then approach the potential subject's parents and give all the information necessary for the parents to make an informed decision whether or not to participate in this study. This process of informing the parents will include answering (or finding answers to) all questions asked by the parents; assuring the parents that the procedure is not necessary to the care of their baby and that declining the study will have no effect on the baby's care; and presenting the consent form with a clear indication to the parents that they can take as much time to consider their decision as they feel they need. No information concerning the subject will be collected unless the parents expressly agree to it and the consent form has been signed. Parents will also be assured that they can withdraw their baby from the study at any time without explanation and that this will not in any way compromise the baby's care.

If the subject's parents decide to participate in the study, the following information will be collected for each imaging session:

Identification number

Date and time

Age

Sex

Reason for admission

Presence of sepsis

Presence of feed intolerance/stool changes (clinical indicators of NEC)

Radiographic changes suggestive of NEC

Recent vasoactive meds

Temperature history

Room temperature

Subject's rectal and axillary temperature

Subject's skin temperatures from incubator/warmer bed probe

Thermal images and settings used (Mid-level temperature, sensitivity) Comments

NB: Although there is no evidence to suggest thermography is affected by timing of feedings or bowel movements or by sleep/wake patterns, we will record the time of last feeding, time of last bowel movement and sleep/wake state at the time of imaging to ensure that subsequent data analysis can identify any previously unrecognized confounding effect of these factors. 


\section{j) Conditions under which a baby would be withdrawn from the study}

Any baby who appears even once not to tolerate the imaging procedure (in particular by exhibiting any of the changes mentioned in paragraph c) will be withdrawn. Babies who are already physiologically unstable will not be imaged unless and until stability returns. Babies who go to the operative room for abdominal surgery will also be withdrawn both to ensure no unnecessary handling of the abdominal wound but also because the incision will generate inflammation and heat, thus rendering thermographic images uninterpretable. Babies in whom a non-treatment decision is made by the parents and team (i.e., full palliative care) will be withdrawn. Parents will have the right to withdraw their baby at any time without prejudice to treatment or any aspect of care.

\section{k) Data handling and data analysis, proposed starting date, and anticipated completion date}

The images will be stored on a personal computer for analysis. The analysis will begin when all the images for all subjects are available.

The proposed starting date for the recording of the thermal images is September 2006. The data collection for the control infants is expected to be completed by September 2007. Final results are expected in December 2007.

\section{l) Analysis of the study}

The investigators will analyze the thermal images from the control population and derive a standard temperature distribution of healthy babies by looking primarily at the degree of asymmetry between contralateral sides of the body. Then, they will analyze the thermal images of babies suspected of having NEC and assess the deviations from the normal population. Different computerized and automated techniques developed in previous work and in the literature on medical thermography will be used for the analysis.

\section{m) Dissemination of results}

Any information derived from this study that contributed to the advancement of knowledge will be communicated to the parents of participating babies. However, since this is a first attempt at imaging NEC and since there is no evidence as yet of a relationship between NEC and thermographic images, it is not expected to be able to communicate definitive results concerning the application of thermography to image NEC. 


\section{Budget:}

There is no sponsor for this study. The medical thermographic equipment will be provided by the investigators. The cost of the study is fully supported by the investigators.

\section{References:}

[1] C. Herry, "Quantitative Assessment of Pain through Clinical Digital Infrared Thermal Imaging", Master's thesis, Department of Systems and Computer Engineering, Carleton University, September 2002.

[2] C. Herry and M. Frize, "Digital Processing Techniques for the Assessment of Pain with Infrared Thermal Images," in Proceedings of the $24^{\text {th }}$ Annual International Conference of the IEEE Engineering in Medicine and Biology Society, 2002.

[3] P. Goodman, M. Murphy, G. Siltanen, M. Kelley, and L. Rucker, "Normal temperature asymmetry of the back and extremities by computer-assisted infrared imaging", Thermology, vol.1, pp. 195-202, 1986.

[4] S. Uematsu, D. Edwin, W. Jankel, J. Kozikowski and M. Trattner, "Quantification of Thermal Asymmetry, part 1: Normal Values and Reproducibility," Journal of Neurosurgery, vol. 69, pp. 552-555, 1988.

[5] M. Frize, C. Herry, N. Scales, "Processing Thermal Images to Detect Breast Cancer and Assess Pain," Proceedings of the 4th Annual IEEE Conference on Information Technology Applications in Biomedicine, UK, 2003. 


\section{Consent Form \\ Assessment of Necrotizing Enterocolitis with Infrared Thermal Imaging}

I acknowledge that the research procedures described above, and of which I have a copy, have been explained to me, and that any questions that I have asked have been answered to my satisfaction. In addition, I know that I may contact my infant's physician if I have further questions either now or in the future.

I have been informed of the alternatives to participation in this study. I understand the possible benefits of joining the research study as well as the possible risks. I have been provided with sufficient time to reflect on my decision to participate. I have been made aware of conditions, which may necessitate the termination of my participation to this research.

I have been assured that personal records relating to this study will be kept confidential and that my baby will not be identified in any publication or presentation of this study. Any personal information about my baby that leaves the hospital will be coded so that my baby cannot be identified.

I understand that I am free to withdraw my baby from the study at any time and there will be no penalty to me or my child. I further understand that if the study is not undertaken or if it is discontinued at any time, the quality of the medical care to my infant will not be affected. I understand that if any knowledge gained from the study becomes available that could influence my decision to continue to participate in this study, I will be promptly informed.

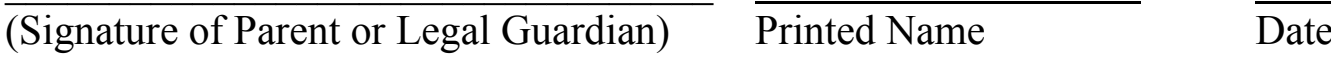

(Signature of Witness)

Printed Name

Date

I HAVE EXPLAINED THIS STUDY TO THE PERSON AUTHORIZED TO SIGN ABOVE AND I AM SATISFIED THAT IT IS UNDERSTOOD.

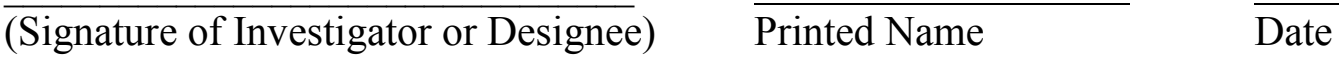

You may contact the Chair of the CHEO Research Ethics Board for information regarding patient's rights in research studies at (XXX) XXX-XXXX ext. XXXX; however this person cannot provide any medical information with regard to this study.

If you have any concerns or questions regarding this study, please contact Dr Erika Bariciak, (XXX) XXX-XXXX extension XXXX; or your baby's neonatologist via the Neonatal Intensive Care Unit, $(\mathbf{X X X )}$ XXX-XXXX.

(Valid until: Oct 8, 2010) 


\section{A.2 CHEO PARENT INFORMATION SHEET \\ Title of the Study: Assessment of Necrotizing Enterocolitis with Infrared Thermal Imaging.}

\section{Background:}

In this study, we will look at the potential use of an infrared thermal camera to detect inflammation and pain associated with necrotizing enterocolitis (NEC). NEC is a serious condition that affects primarily premature newborns. Its cause is still unclear and its prevention difficult. NEC affects the bowel, leading to inflammation, necrosis (dead tissue) and perforation of areas of the bowel. The mortality rate has improved in recent years but is still about $15 \%$ and survivors may suffer significant complications including long-term damage to bowel function.

NEC is also believed to produce significant pain due to the inflammation of the bowel. As newborns are believed to feel pain more intensely than older persons because their protective mechanisms are not fully developed, recognition and treatment of pain is very important in this population.

Currently, only abdominal x-ray imaging is used systematically to diagnose NEC, as the other techniques such as abdominal sonography and more recently MRI do not allow the doctors to specifically identify NEC.

The skin temperature is an important clinical parameter because it varies with the core temperature and is closely related to how well the body works. Monitoring the skin and core temperature of infants is especially important since the regulation of their body temperature is still immature and even small changes of temperature may be precious indicators of a disease. Although skin temperature is not proven to be related to the initial diagnosis of NEC it often appears elevated to the touch, presumably because of the underlying often severe inflammation. Our hypothesis is that thermographic imaging will be able to detect even small changes in temperature in localized areas of the abdomen. These would not necessarily be detectable by human touch and it is expected that the camera will be able to detect changes from normal much sooner than by touch.

Infrared Thermal Imaging is a simple imaging procedure that uses an infrared camera to take images of the temperature of the body. It does not require any injection and does not send any radiation such as X-rays. The body naturally gives off infrared radiation (or heat) and an infrared camera simply takes a picture showing how much infrared radiation (or heat) is given off. The resulting picture is a thermal or temperature map of the body. Infrared Thermal Imaging may provide valuable information about the initiation and progression of functional abnormalities or pathologies, such as the inflammation and pain associated with NEC. 


\section{Procedure:}

We wish to enroll your infant in this study. A nurse will remove the clothes covering the abdomen of your baby inside the incubator. If your baby is on an overbed warmer, the clothing will be removed while the heater is still on. Then your baby will be gently rolled out of the incubator, or the overbed heater will be turned off, for about 60-90 seconds so that we can take a few thermal images of your baby's abdomen. A nurse will maintain your baby still during each image recording (these take only a moment) to ensure that the recording is good. Your baby will then be placed back in the incubator, or the overbed warmer will be turned back on, and the nurse will put the clothes back on if necessary.

There are no known risks associated with infrared thermal imaging. However, the handling of premature babies may cause changes in heart rate, blood pressure, blood oxygen saturation or distress level. During the imaging procedure, the nurse will therefore monitor your baby for any significant changes that would require the termination of the imaging procedure. Although this procedure should be absolutely painless, the nurse will also monitor for the pain level of your baby as a precaution and decide whether or not the imaging has to be stopped.

If your baby's temperature drops below acceptable levels of temperature, your baby will be immediately placed back in the incubator or the overbed heater will be turned back on. We may perform this procedure up to three times to determine the stability of the temperature distribution of your baby's abdomen over several days.

We wish to consider two distinct types of population for our study. The first part of the study will consider healthy premature infants and the second part will consider infants who present signs of NEC. From the analysis of the two populations, we will devise a decision-support system that could help the physician make informed decisions about the diagnosis and management of newborns suspected of having NEC.

If the results are published, your infant will not be identified in any way.

At the conclusion of the study, you will be given a summary of the results of the study.

Your decision to participate or not in this study will not affect the care your baby receives at the Children's Hospital of Eastern Ontario. No money is being offered for your baby's involvement in this study.

We will inform you of any new information that might influence your decision to continue to participate in this research project.

If you choose not to participate or decide to withdraw from the study, normal standard medical care will continue to be provided. 


$\begin{array}{ll}\text { APPENDIX B } & \\ \text { BLB } 1 \text { LIST OF ACRONYMS } & \\ \text { FFT } & \text { Fastremely low birth weight } \\ \text { GI } & \text { Gastrointestinal Tract } \\ \text { LLQ } & \text { Left lower quadrant } \\ \text { LUQ } & \text { Left upper quadrant } \\ \text { MSE } & \text { Mean Square Error } \\ \text { NEC } & \text { Necrotizing Enterocolitis } \\ \text { QTW } & \text { Quad-to-Whole } \\ \text { RLQ } & \text { Right lower quadrant } \\ \text { ROI } & \text { Region of Interest } \\ \text { RUQ } & \text { Right upper quadrant } \\ \text { VLBW } & \text { Very Low Birth Weight }\end{array}$




\section{Bibliography}

[1] P. W. Lin, T. R. Nasr , B. J. Stoll, “Necrotizing Enterocolitis: Recent Scientific Advances in Pathophysiology and Prevention," Seminars in Perinatology, vol. 32, no. 2, pp. 7082, Apr. 2008.

[2] M. Henry, R. Moss, "Necrotizing enterocolitis," The Annual Review of Medicine, vol. 60, pp. 111-124, 2009.

[3] J. Neu, M. Walter, "Recent Developments in Necrotizing Enterocolitis," Journal of Parenteral and Enteral Nutrition, vol. 36, no. 1, pp. 30S-35S, 2012.

[4] M. Petrosyan, Y. S. Guner, M. Williams, A. Grishin, H. R. Ford, "Current concepts regarding the pathogenesis of necrotizing enterocolitis," Pediatric Surgery International, vol. 25, no. 4, pp. 309-318, Apr. 2009.

[5] P. Lin, J. Stoll, "Necrotizing enterocolitis," The Lancet, vol. 368(9543), pp. 12711283, Oct. 2006.

[6] K. Schnabl, J. Van Aerde, A. Thomson, M. Clandinin, "Necrotizing enterocolitis: A multifactorial disease with no cure," The World Journal of Gastroenterology, vol. 14, no. 14, pp. 2142 - 2161, Apr. 2008.

[7] P. Gordon, J. Swanson, J. Attridge, R. Clark, "State Of The Art Emerging trends in acquired neonatal intestinal disease: is it time to abandon Bell's criteria?," Journal of Perinatology, vol. 27, pp. 661 - 671, July 2007. 
[8] S. Oh, C. Young, N. Gravenstein, S. Islam, J. Neu, "State-of-the-Art Monitoring technologies in the neonatal intensive care unit: implications for the detection of necrotizing enterocolitis," Journal of Perinatology, vol. 30, pp. 701-708, 2010.

[9] M. Epelman, A. Daneman, O. Navarro, I. Morag, A. Moore, J. Kim, R. Faingold, G. Taylor, T. Gerstle, "Necrotizing Enterocolitis: Review of State-of-the-Art Imaging Findings with Pathologic Correlation," RadioGraphics, vol. 27, no. 2, pp. 285 - 305, Mar. 2001.

[10] B. Frost, T. Jilling, M. Caplan, "The importance of pro-inflammatory signaling in neonatal NEC," Seminars in Perinatology, vol. 32, no. 2, pp. 100-106, Apr. 2008.

[11] C. Young, R. Sharma, M. Handfield, V. Mai, J. Neu, "Biomarkers for Infants at Risk for Necrotizing Enterocolitis: Clues to Prevention?," Pediatric Research, vol. 65, no. $5 \mathrm{Pt}$ 2, pp. 91R-97R, May 2009.

[12] B. F. Jones, "A Reappraisal of the Use of Infrared Thermal Image Analysis in Medicine," IEEE Transactions on Medical Imaging, vol. 17, no. 6, pp. 1019 - 1027, Dec. 1998.

[13] M. Anbar, "Clinical thermal imaging today," IEEE Engineering in Medicine and Biology, vol. 17, no. 4, pp. 25-33, Jul. 1998.

[14] T. Ishigaki, M. Ikeda, H. Asai, S. Sakuma, "Forehead back thermal ratio for the interpretation of infrared imaging of spinal cord lesions and other neurological disorders," Thermology International, vol. 3, pp. 101-107, 1989. 
[15] A. Merla, G. L. Romani, "Functional Infrared Imaging in Medicine: A Quantitative Diagnostic Approach," in Proc. of the Annual International Conference of the IEEE Engineering in Medicine and Biology Society, New York, NY, USA, 2006, pp. 224-227.

[16] J. F. Head, C. A. Lipari, R. L. Elliot, “Computerized image analysis of digitized infrared images of breasts from a scanning infrared imaging system," in Proc. of SPIE Infrared Technology and Applications Conference, San Diego, CA, USA, 1998, pp. 290-294.

[17] M. Frize, C. Herry, R. Roberge, "Processing of Thermal Images to Detect Breast Cancer: Comparison with Previous Work," in Proc. of the Annual International Conference of the IEEE Engineering in Medicine and Biology Society, Houston, TX, USA, 2002, pp. 1159-1160.

[18] M. Frize, C. Adea, P. Payeur, G. Di Primio, J. Karsh and A. Ogungbemile, "Detection of rheumatoid arthritis using infrared imaging," in Proc. of SPIE Medical Imaging Conference, Lake Buena Vista, FL, USA, 2011.

[19] E. F. Ring, "Quantitative thermal imaging in rheumatology," in Proc. of the Annual International Conference of the IEEE Engineering in Medicine and Biology Society, Baltimore, MD, USA, 1994.

[20] A. K. Saxena, G. H. Willital, "Infrared thermography: Experience from a decade of pediatric imaging," European Journal of Pediatrics, vol. 167, no. 7, pp. 757-764, 2008.

[21] H. E. Rice, C. L. Hollingsworth, E. Bradsher, M. E. Danko, S. Crosby, R. N. Goldberg, D. T. Tanaka, R. B. Knobel, "Infrared Thermal Imaging (Thermography) of the Abdomen in Extremely Low Birthweight Infants," The Journal of Surgical Radiology, vol. 1, no. 2, pp. 82-89, July 2010. 
[22] J. Neu, W. A. Walker, "Necrotizing enterocolitis," The New England Journal of Medicine, vol. 364, no. 3, pp. 255-264, 2011.

[23] S. Gephart, J. McGarth, J. Effken, M. Halpern, "Necrotizing enterocolitis risk: state of the science," Advances in Neonatal Care, vol. 12, no. 2, pp. 77-87, Apr. 2012.

[24] J. Neu, M. Mshvildadze, V. Mai, "A roadmap for understanding and preventing necrotizing enterocolitis," Current Gastroenterology Reports, vol. 10, no. 5, pp. 450457, Oct. 2008.

[25] A. Thompson, M. J. Bizzarro, "Necrotizing Enterocolitis in Newborns: Pathogenesis, Prevention and Management," Drugs, vol. 68, no. 9, pp. 1227-1238, 2008.

[26] M. Bell, J. Ternberg, R. Feigin, J. Keating, R. Marshall, L. Barton, T. Brotherton, “Neonatal Necrotizing Enterocolitis: Therapeutic Decisions Based upon Clinical Staging," Annals of Surgery, vol. 187, no. 1, pp. 1-7, Jan. 1978.

[27] M. Walsh, R. M. Kliegman, "Necrotizing enterocolitis: treatment based on staging criteria," Pediatric Clinics of North America, vol. 3, no. 1, pp. 179-201, Feb. 1986.

[28] B. K. Patel, J. S. Shah, "Necrotizing Enterocolitis in Very Low Birth Weight Infants: A Systemic Review," ISRN Gastroenterology, pp. 1-7, Sept. 2012.

[29] S. Miller, J. Seibert, D. Kinder, A. Wilson, "Use of ultrasound in the detection of occult bowel perforation in neonates," Journal of Ultrasound in Medicine, vol. 12, no. 9, p. 531-535, Sept. 1993. 
[30] A. Franco, F. Ramji, "Utility of abdominal sonography to diagnose necrotizing enterocolitis," European Journal of Radiology Extra, vol. 65, no. 1, pp. 13-16, Jan. 2008.

[31] E. Maalouf, A. Fagbemi, P. Duggan, S. Jayanthi, H. Lewis, A. Fletcher, K. Lakhoo, A. Edwards, "Magnetic Resonance Imaging of Intestinal Necrosis in Preterm Infants," American Academy of Pediatrics, vol. 105, no. 3 Pt 1, pp. 510-514, Mar. 2000.

[32] M. Schlessinger, Infrared Technology Fundamentals Second Edition, New York: Marcel Dekker, Inc., 1995, pp. 1-25.

[33] R. Knobel, B. Guenther, H. Rice, "Thermoregulation and Thermography in Neonatal Physiology and Disease," Biological Research for Nursing, vol. 13, no. 3, pp. 274-282, May 2011.

[34] J. Steketee, "Spectral emissivity of skin and pericardium," Physics in Medicine and Biology, vol. 15, no. 5, pp. 686-694, 1973.

[35] D. Watmough, P. Fowler, R. Oliver, "The Thermal Scanning of a Curved Isothermal Surface: Implications for Clinical Thermography," Physics in Medicine and Biology, vol. 15, no. 1, pp. 1-8, 1970.

[36] M. Anbar, "Objective Assessment of Clinical Computerized Thermal Images," in Proc. of SPIE Medical Imaging Conference, San Jose, CA, USA, June 1991, pp. 479-484.

[37] Humamatsu Photonics K.K., "Characteristics and use of Infrared Detectors," [Online]. Available: http://www.slac.stanford.edu/grp/arb/tn/arbvol5/AARD460.pdf. [Jan $10,2014]$ 
[38] R. J. Keyes, Optical and infrared detectors, Berlin, New York: Springer-Verlag, 1977.

[39] S. Donati, Photodetectors: Devices, Circuits, and Applications, Upper Saddle River: Prentice Hall, 2000.

[40] C. H. Jones, "Medical thermography," IEE Proceedings on Physical Science, Measurement and Instrumentation, Management and Education, vol. 134, No. 2, Feb. 1987, pp. 225-236.

[41] C. L. Herry, "Quantitative assessment of pain through clinical digital infrared thermal imaging." M.A.Sc thesis, Carleton University, Canada, 2002.

[42] P. Norton, S. Horn, J. G. Pellegrino, P. Perconti, "Infrared Detectors and Detector Arrays," in Medical Infrared Imaging, Boca Raton, FL: CRC Press, 2008, ch. 37, pp. 126.

[43] A. Rogalski, Infrared Detectors, Amsterdam: Gordon \& Breach Science Publishers, 2000.

[44] K. Weir, "Detectors," in The Handbook of Laser Technology and Applications, Philadelphia, USA: Institute of Physics Publishing Ltd, 2004, pp. 1509-1522.

[45] E. F. Ring, K. Ammer, "The Technique of Infra red Imaging in Medicine," Thermology International, vol. 10, no. 1, pp. 7-14, 2000.

[46] D. Kennedy, T. Lee, D. Seely, "A Comparative Review of Thermography as a Breast Cancer Screening Technique," Integrative Cancer Therapies, vol. 8, no. 1, pp. 9-16, 2009. 
[47] K. Ammer, E. F. Ring, "Standard Procedures for Infrared Imaging in Medicine," in Medical Systems and Devices, Boca Raton, FL: CRC Press, 2006, ch. 36, pp. 1-14.

[48] S. Uematsu, D. Edwin, W. Jankel, J. Kozikowski, M. Trattner, "Quantification of thermal asymmetry. Part 1: Normal values and reproducibility," Journal of Neurosurgery, vol. 69, no. 4, pp. 552-5, Oct. 1988.

[49] N. Niu, P. Lui, J. Hu, C. Ting, Y. Yin, Y. Lo, L. Liu, T. Lee, "Thermal symmetry of skin temperature: normative data of normal subjects in Taiwan," Journal of the Chinese Medical Association, vol. 64, no. 8, pp. 459-68, Aug. 2001.

[50] B. Wiecek, R. Danych, Z. Zwolenik, A. Jung, J. Zuber, "Advanced thermal image processing for medical and biological applications," in Proc. of the Annual International Conference of the IEEE Engineering in Medicine and Biology Society, 2001, vol. 3, pp. 2805 - 2807, 2001.

[51] J. Montoro, M. Anbar, "New modes of data handling in computerized thermography," in Proc. of the Annual International Conference of the IEEE Engineering in Medicine and Biology Society, New Orleans, LA, USA, 1988.

[52] J. Head, F. Wang, C. Lipari, R. Elliott, "The Important Role of Infrared Imaging in Breast Cancer," IEEE Engineering in Medicine and Biology Magazine, vol. 19, no. 3, pp. 52-57, 2000.

[53] C. Lipari, J. Head, "Advanced infrared image processing for breast cancer risk assessment," in Proc. of the Annual International Conference of the IEEE Engineering in Medicine and Biology Society, Chicago, IL, USA, 1997, pp. 673-676. 
[54] M. Frize, C. Herry, N. Scales, "Processing thermal images to detect breast cancer and assess pain," in Proc. of IEEE EMBS Special Topic Conference on Information Technology Applications in Biomedicine, 2003, pp. 234-237.

[55] K. Mabuchi, T. Chinzei, I. Fujimasa, S. Haeno, Y. Abe, T. Yonezawa, "An imageprocessing program for the evaluation of asymmetrical thermal distributions," in Proc. of the Annual International Conference of the IEEE Engineering in Medicine and Biology Society, Chicago, IL, USA, 1997, pp. 725-728.

[56] V. Vavilov, E. Vavilova, D. Popov, "Statistical analysis of human body temperature asymmetry as the basis for detecting pathologies by means of IR thermography," in Proc. of SPIE Thermosense Conference, Orlando, FL, USA, 2001.

[57] T. Jakubowska, B. Wiecek, M. Wysocki, C. Drews-Peszynski, "Thermal signatures for breast cancer screening comparative study," in Proc. of the Annual International Conference of the IEEE Engineering in Medicine and Biology Society, Cancun, Mexico, 2003, pp. 1117-1120.

[58] J. Head, C. Lipari, F. Wang, J. Davidson, R. Elliot, "Application of second generation infrared imaging with computerized image analysis to breast cancer risk assessment," in Proc. of the Annual International Conference of the IEEE Bridging Disciplines for Biomedicine, Amsterdam, 1996, pp. 2093-2094.

[59] J. Keyserlingk, P. Ahlgren, E. Yu, N. Belliveau, "The contribution of currently available high resolution infra-red imaging to the detection of stage I and II breast cancer," in Proc. of the Annual International Conference of the IEEE Engineering in Medicine and Biology Society, Chicago, IL, USA, 1997, pp. 662-669. 
[60] J. Keyserlingk, P. Ahlgren, E. Yu, N. Belliveau, M. Yassa, "Functional Infrared Imaging of the Breast," IEEE Engineering in Medicine and Biology Magazine, vol. 19, no. 3, pp. $30-41,2000$.

[61] A. Collins, E. Ring, J. Cosh, P. Bacon, "Quantitation of thermography in arthritis using multi-isothermal analysis. I. The thermographic index," Ann. Rheum. Dis., vol. 33, no. 2, pp. 113-115, 1974.

[62] E. Ring, A. Collins, P. Bacon, J. Cosh, "Quantitation of thermography in arthritis using multi-isothermal analysis. II. Effect of nonsteroidal anti-inflammatory therapy on the thermographic index," Ann. Rheum. Dis., vol. 33, no. 4, pp. 353-356, 1974.

[63] M. Anbar, "Dynamic area telethermometry and its clinical applications," in Proc. of SPIE Thermosense Conference on Thermal Sensing and Imaging Diagnostic Applications, Orlando, FL, USA, 1995, pp. 312-322.

[64] I. Fujimas, "Pathophysiological expression and analysis of far infrared thermal images," IEEE Engineering in Medicine and Biology Magazine, vol. 17, no. 4, pp. 3442, 1998.

[65] A. Merla, L. Di Donato, S. Di Luzio, G. Farina, S. Pisarri, M. Proietti, F. Salsano, G. Luca Romani, "Infrared functional imaging applied to Raynaud's phenomenon," IEEE Engineering in Medicine and Biology Magazine, vol. 21, no. 6, pp. 73-79, 2002.

[66] A. Merla, G. Romani, S. Di Luzio, L. Di Donato, G. Farina, M. Proietti, S. Pisarri, S. Salsano, "Raynaud's phenomenon: Infrared functional imaging applied to diagnosis and drug effects," Int. J. Immunopathol. Pharmacol., vol. 15, no. 1, pp. 41-52, 2002. 
[67] Y. Fujiwara, T. Inukai, Y. Aso, Y. Takemura, "Thermographic measurement of skin temperature recovery time of extremities in patients with type 2 diabetes mellitus," Exp. Clin. Endocrinol. Diabetes, vol. 108, no. 7, pp. 463-469, 2000.

[68] H. Zotter, R. Kerbl, S. Gallistl, H. Nitsche, M. Borkenstein, "Rewarming index of the lower leg assessed by infrared thermography in adolescents with type 1 diabetes mellitus," J. Pediatr. Endocrinol. Metab., vol. 16, no. 9, pp. 1257-1262, 2003.

[69] Y. Ohashi, I. Uchida, "Applying dynamic thermography in the diagnosis of breast cancer," IEEE Engineering in Medicine and Biology Magazine, vol. 19, no. 3, pp. 42-51, 2000.

[70] Y. Ohashi, I. Uchida, "Some considerations on the diagnosis of breast cancer by thermography in patients with nonpalpable breast cancer," in Proc. of the Annual International Conference of the IEEE Engineering in Medicine and Biology Society, Chicago, IL, USA, 1997, pp. 670-672.

[71] M. Anbar, "Dynamic area telethermometry (DAT)," in Proc. of the Annual International Conference of the IEEE Engineering in Medicine and Biology Society, Chicago, IL, USA, 2000, pp. 1635-1638.

[72] J. Montoro, M. Anbar, "Visualization and analysis of dynamic thermographic changes," in Proc. of Visualization in Biomedical Computing, Atlanta, GA, USA, 1990, pp. 486-489.

[73] M. Unser, H. Van Hamme, E. Van Denhaute, J. Cornelis, "Karhunen-Loeve analysis of dynamic sequences of thermographic images for early breast cancer detection," in 
Proc. of IEEE Computer Society Conference on Computer Vision and Pattern Recognition, Ann Arbor, MI, USA, 1988, pp. 592-596.

[74] M. Haidekker, "Texture Analysis," in Advanced Biomedical Image Analysis, Hoboken, NJ: John Wiley \& Sons, Inc., 2011, pp. 236 - 275.

[75] I. Young, J. Gerbrands, L. Van Vliet, Fundamentals of Image Processing. Netherlands: Delft University of Technology, 1998.

[76] C. Herry, R. Goubran, M. Frize, "Segmentation of Infrared Images Using Cued Morphological Processing of Edge Maps," in Proc. of IEEE Instrumentation and Measurement Technology Conference, Warsaw, Poland, 2007.

[77] C. Herry, "Segmentation and extraction of regions of interest for automated detection of anomalies in clinical thermal infrared images," Ph.D thesis, Carleton University, Canada, 2008.

[78] C. Herry, R. Goubran, M. Frize, "Improving the Detection and Localization of Anatomical Landmark Points in Infrared Images Using Symmetry and Region Specific Constraints," in Proc. of IEEE Instrumentation and Measurement Technology Conference, Victoria, BC, Canada, 2008, pp. 1306-1311.

[79] B. Wiecek, S. Zwolenik, A. Jung, J. Zuber, "Advanced thermal, visual and radiological image processing for clinical diagnostics," in Proc. of IEEE Engineering in Medicine and Biology and the Annual Fall Meeting of the Biomedical Engineering Society, Atlanta, GA, USA, 1999, pp. 1108. 
[80] W. Snyder, H. Qi, R. Elliot, J. Head, C. Wang, "Increasing the effective resolution of thermal infrared images," IEEE Engineering in Medicine and Biology Magazine, vol. 19, no. 3, pp. 63-70, 2000.

[81] H. Qi, W. Snyder, J. Head, R. Elliott, “Detecting Breast Cancer from Infrared Images by Asymmetry Analysis," in Proc. of the Annual International Conference of the IEEE Engineering in Medicine and Biology Society, Chicago, IL, USA, 2000, pp. 127-128.

[82] C. Bao-ping, Z. Ma, "Automated Image Segmentation and Asymmetry Analysis for Breast Using Infrared Images," in Proc. of International Workshop on Education Technology and Training, and International Workshop on Geoscience and Remote Sensing, Shanghai, China, 2008, pp. 410-413.

[83] E. Ring, K. Ammer, "Infrared thermal imaging in medicine," Physiol. Meas., vol. 33, no. 3, pp. 33-46, 2012.

[84] C. Herry, M. Frize, E. Bariciak, "Assessment of Abdominal Skin Temperature Change in Premature Newborns with NEC Compared to Healthy Controls," in Proc. of the International Federation for Medical and Biological Engineering, Budapest, Hungary, 2012, pp. 191-194.

[85] J. Semmlow, Biosignal and Biomedical Image Processing, New York: Marcel Dekker, Inc., 2004, pp. 1-40.

[86] J. Parker, R. Kenyon, D. Troxel, "Comparison of Interpolating Methods for Image Resampling," IEEE Transactions on Medical Imaging, vol. 2, no. 1, pp. 31-39, 1983.

[87] A. Weeks, "Spatial Filtering and Fourier Frequency Methods," in Fundamentals of Electronic Image Processing, Bellingham, WA: Wiley - IEEE Press, 1996, pp. 121-172. 
[88] M. Haidekker, "Fractal Approaches to Image Analysis," in Advanced Biomedical Image Analysis, Hoboken, NJ: John Wiley \& Sons, Inc., 2011, pp. 310-349.

[89] N. Otsu, "A Threshold Selection Method from Gray-Level Histograms," IEEE Transactions on Systems, Man, and Cybernetics, vol. 9, no. 1, pp. 62-66, 1979.

[90] E. Pisano, S. Zong, B. Hemminger, M. DeLuca, R. Johnston, K. Muller, M. Braeuning, S. Pizer, "Contrast limited adaptive histogram equalization image processing to improve the detection of simulated spiculations in dense mammograms," J. Digital Imaging, vol. 11, no. 4, pp. 193-200, 1998.

[91] Y. Zhao, N. Georganas, E. Petriu, "Applying Contrast-limited Adaptive Histogram Equalization and integral projection for facial feature enhancement and detection," in Proc. of IEEE Instrumentation and Measurement Technology Conference, Austin, TX, USA, 2010, pp. 861-866.

[92] S. Pizer, R. Johnston, J. Ericksen, B. Yankaskas, K. Muller, “Contrast-limited adaptive histogram equalization: speed and effectiveness," in Proc. of Visualization in Biomedical Computing, Atlanta, GA, USA, 1990, pp. 337-345.

[93] R. Nur, M. Frize, "Image processing of infrared thermal images for the detection of necrotizing enterocolitis," in Proc. of SPIE Medical Imaging Conference, Lake Beuna Vista, FL, USA, 2013.

[94] M. Frize, R. Nur, E. Bariciak, C. Herry, "Infrared Imaging and Classification of Neonates with Necrotizing Enterocolitis," in Proc. of World Congress on Medical Physics and Biomedical Engineering, Beijing, China, 2012. 
[95] R. Haralick, K. Shanmugan, I. Dinstein, "Textural Features for Image Classification," IEEE Transactions on Systems, Man, and Cybernetics, vol. 3, no. 6, pp. 610-621, 1973.

[96] D. Clausi, "An analysis of co-occurrence texture statistics as a function of grey level quantization," Can. J. Remote Sensing, vol. 28, no. 1, pp. 45-62, 2002.

[97] B. Wiecek, M. Strzelecki, T. Jakubowska, M. Wysocki, C. Drews-Peszynski, "Advanced Thermal Image Processing," in Medical Infrared Imaging, Boca Raton, FL: CRC Press, 2008, ch. 28, pp. 1-13.

[98] S. Dravida, J. Woods, W. Shen, "A comparison of image filtering algorithms," in Proc. of IEEE International Conference on ICASSP, 1984, pp. 259-262.

[99] MathWorks, Inc., "Matlab Product Documentation: Lillietest," [Online]. Available: http://www.mathworks.com/help/toolbox/stats/lillietest.html. [Jan 10, 2014].

[100] StatSoft Inc., "Electronic Statistics Textbook Glossary," [Online]. Available: http://www.statsoft.com/Textbook/Statistics-Glossary. [Jan 10, 2014].

[101] MathWorks, Inc., "Matlab Product Documentation: Kruskal-Wallis Test," [Online]. Available: http://www.mathworks.com/help/toolbox/stats/kruskalwallis.html. [Jan 10, 2014].

[102] MathWorks, Inc., "Matlab Product Documentation: Wilcoxon Rank Sum Test," [Online]. Available: http://www.mathworks.com/help/toolbox/stats/ranksum.html. [Jan 10, 2014].

[103] M. Frize, R. Walker, C. Herry, E. Bariciak, "Research Ethics Proposal," Aug. 2006. 
[104] M. Frize, R. Walker, C. Herry, E. Bariciak, "CHEO Parent Information Sheet," Aug. 2006. 